\title{
MEASUREMENT OF THE BAND BENDING AND SURFACE DIPOLE AT CHEMICALLY \\ FUNCTIONALIZED SI(111)/VACUUM INTERFACES
}

\author{
Thesis by \\ David Charles Gleason-Rohrer
}

In Partial Fulfillment of the Requirements for the Degree

of

Doctor of Philosophy

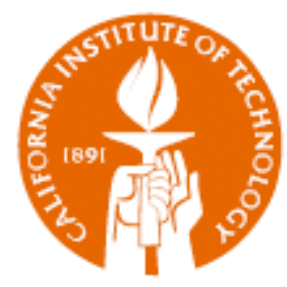

\section{CALIFORNIA INSTITUTE OF TECHNOLOGY \\ Pasadena, California \\ 2014}

(Defended September 30, 2013) 
(C) 2014

David Charles Gleason-Rohrer

All Rights Reserved 


\section{ACKNOWLEDGEMENTS}

I am very pleased to be presenting this thesis and all of the work that it represents, and I am truly grateful to all of the people who have helped to make it a reality.

First and foremost I would like to thank my advisor Professor Nathan Lewis. Nate works tirelessly to support and strengthen the research done within the Lewis group. Nate has given me the freedom to pursue my scientific interests and ideas. His patience has allowed me to develop my work into something of which I am extremely proud. His guidance and direction have shaped this project whether in discussions and group meetings or in my own literature searches stumbling across old papers of his that are still as relevant as ever. Nate has been very accommodating in allowing me "a bit" of extra time to finish, and as a result I feel that I have been able to reach a satisfying conclusion to my research. For this I am extremely grateful.

Thanks go to Bruce Brunschwig, the Director of the Molecular Materials Research Center, for invaluable contributions to my development as a researcher and a scientist. In my role as a Graduate Laboratory Assistant I worked with Bruce to maintain the instrumentation of the MMRC as well as train users and facilitate their work. Bruce literally taught me the nuts and bolts of ultrahigh vacuum systems. He also helped immensely by talking through with me much of the theory presented in this thesis. Bruce forced me to hone my understanding of the fundamentals of X-ray Photoelectron Spectroscopy, and he forbade any fuzzy thinking or hand waving. 
I acknowledge the remaining members of my committee: Harry Gray, Jackie Barton, and William Goddard. I had the good fortune of taking Ch 132, Biophysics of Macromolecules, with Dr. Barton and Ch 120, Nature of the Chemical Bond, with Dr. Goddard. Both were excellent courses that I would recommend to others. I regret that I never took a course from Dr. Gray, however his passion for science has been infectious. I am very thankful for the input my committee has given me over the years.

I would like to thank the members of the Lewis group for friendship, camaraderie, and scientific insight. Particular thanks go to Patrick Hurley who taught me electrochemistry, and Lauren Webb who introduced me to silicon surface chemistry as well as the use of the XPS. I have worked with a wonderful team of GLAs over the years to keep the instruments of the MMRC, running including David Knapp, Tony Fitch, Joseph Beardslee, and Judy Lattimer. Other group members that have been particularly helpful include Kate Plass, David Michalak, Edgardo García-Berríos, Marc Woodka, Craig Wiggenhorn, Ronald Grimm, Erik Johansson, Gregory Kimball, and Mike Rose. Special thanks also to Gray group member James Blakemore for independently reproducing some of my results.

My parents, David and Brenda Gleason, have been wonderfully supportive throughout my entire education. I am so thankful that you both encouraged me early with puzzles. I view this work as an attempt, hopefully successful, at solving the greatest puzzle of my life. Thank you Mom and Dad for all of the sacrifices you have made over the years on my behalf and for teaching me all the most important things in life like how to be happy, healthy, and full of love. 
Finally, I acknowledge my loving wife Dr. Gwen Gleason-Rohrer. Gwen has been with me through this every step of the way. Through the many setbacks in lab, Gwen has been able to keep me positive and moving forward. Thank you for believing in me and encouraging me. Thank you for inspiring me and motivating me. Thank you for making a family with me. I love you. 
The core-level energy shifts observed using X-ray photoelectron spectroscopy (XPS) have been used to determine the band bending at $\mathrm{Si}(111)$ surfaces terminated with $\mathrm{Si}-\mathrm{Br}, \mathrm{Si}-\mathrm{H}$, and $\mathrm{Si}-\mathrm{CH}_{3}$ groups, respectively. The surface termination influenced the band bending, with the Si $2 p_{3 / 2}$ binding energy affected more by the surface chemistry than by the dopant type. The highest binding energies were measured on $\mathrm{Si}(111)-\mathrm{Br}$ (whose Fermi level was positioned near the conduction band at the surface), followed by $\mathrm{Si}(111)-\mathrm{H}$, followed by $\mathrm{Si}(111)-\mathrm{CH}_{3}$ (whose Fermi level was positioned near mid-gap at the surface). Si(111)$\mathrm{CH}_{3}$ surfaces exposed to $\mathrm{Br}_{2}(\mathrm{~g})$ yielded the lowest binding energies, with the Fermi level positioned between mid-gap and the valence band. The Fermi level position of $\mathrm{Br}_{2}(\mathrm{~g})$ exposed $\mathrm{Si}(111)-\mathrm{CH}_{3}$ was consistent with the presence of negatively charged brominecontaining ions on such surfaces. The binding energies of all of the species detected on the surface $(\mathrm{C}, \mathrm{O}, \mathrm{Br})$ shifted with the band bending, illustrating the importance of isolating the effects of band bending when measuring chemical shifts on semiconductor surfaces. The influence of band bending was confirmed by surface photovoltage (SPV) measurements, which showed that the core levels shifted toward their flat-band values upon illumination. Where applicable, the contribution from the X-ray source to the SPV was isolated and quantified. Work functions were measured by ultraviolet photoelectron spectroscopy (UPS), allowing for calculation of the sign and magnitude of the surface dipole in such systems. The values of the surface dipoles were in good agreement with previous measurements as well as with electronegativity considerations. The binding energies of the adventitious carbon signals were affected by band bending as well as by the surface dipole. A model of band bending in which charged surface states are located exterior to the surface 
dipole is consistent with the XPS and UPS behavior of the chemically functionalized

Si(111) surfaces investigated herein. 


\section{TABLE OF CONTENTS}

Acknowledgements ...............................................................................ii

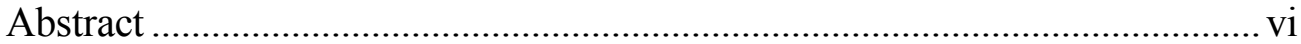

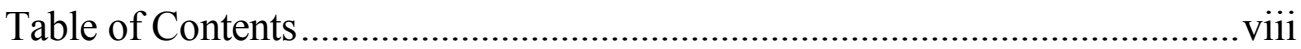

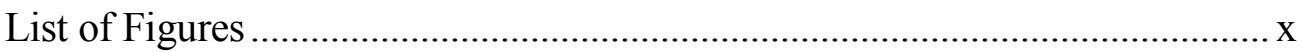

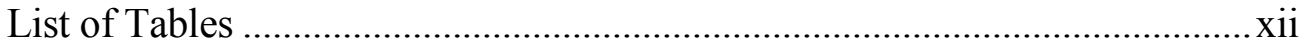

Chapter 1: Silicon Surfaces and Band Bending

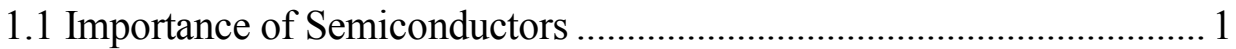

1.2 Semiconductor Physics ...................................................................... 2

1.3 History of Semiconductor Device Development and Surface States .... 6

1.4 Silicon Surface Chemistry and Analysis ........................................... 13

1.4.1 Molecular Modification ............................................................... 13

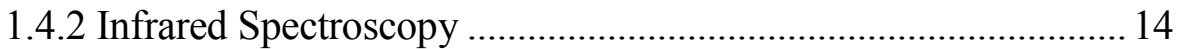

1.4.3 Scanning Tunneling Microscopy .............................................. 16

1.4.4 X-ray Photoelectron Spectroscopy ........................................... 19

1.5 Band Bending Determination by XPS …........................................ 21

1.6 Electrochemistry, Band Bending, and Surface States ......................... 28

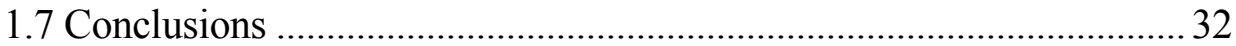

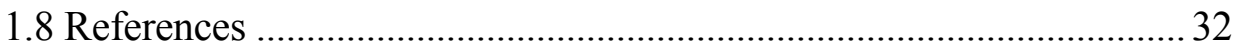

Chapter 2: Measurement of the Band Bending and Surface Dipole at Chemically Functionalized Si(111)/Vacuum Interfaces

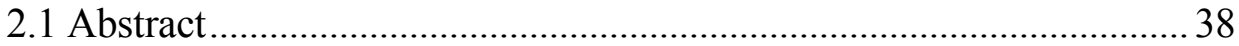

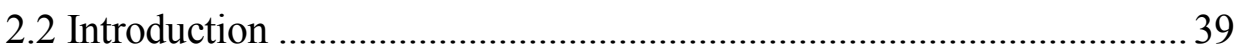

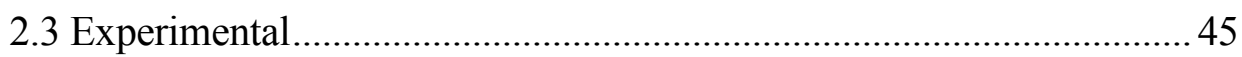

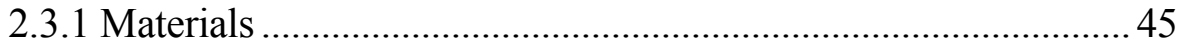

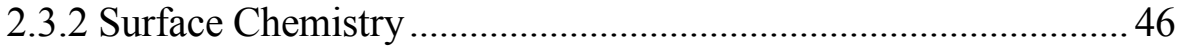

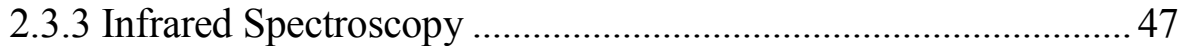

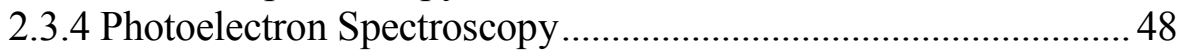

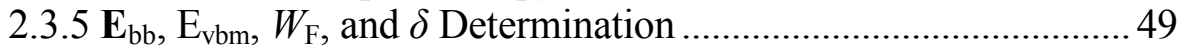

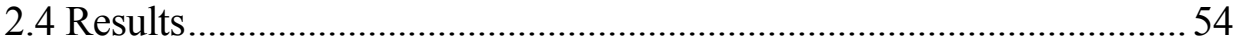

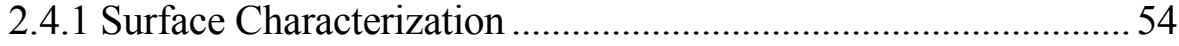




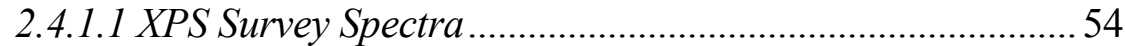

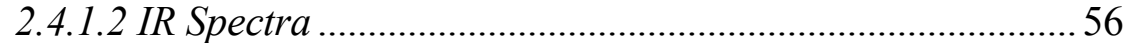

2.4.2 Binding Energy Shifts of Si 2p Peaks in the Dark ..................... 58

2.4.2.1 Moderate Dopant Density................................................ 58

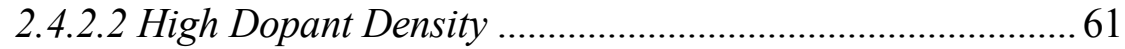

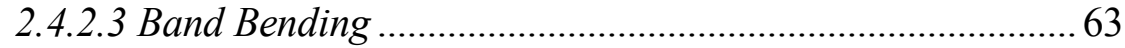

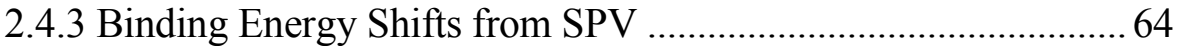

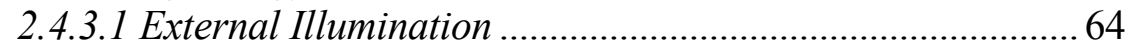

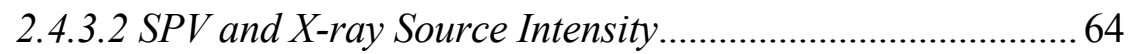

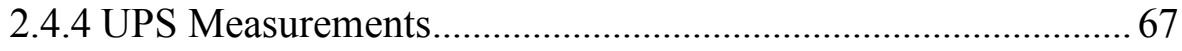

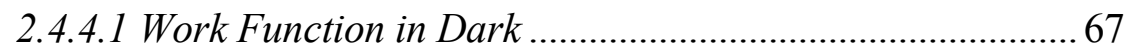

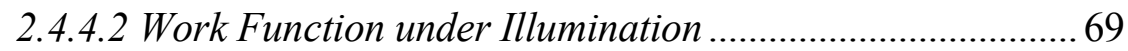

2.4.5 Binding Energy Shifts of C 1s Peaks ........................................69

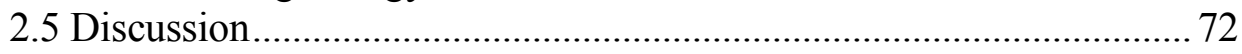

2.5.1 Band Bending and Qss .............................................................. 72

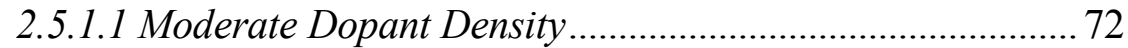

2.5.1.2 High Dopant Density ......................................................... 73

2.5.2 Surface Dipole and Qss .......................................................... 74

2.5.2.1 Electronegativity ........................................................... 74

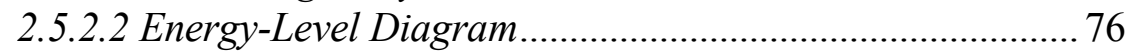

2.5.2.3 Surface Dipole vs. Terminal Si Electron Density............... 78

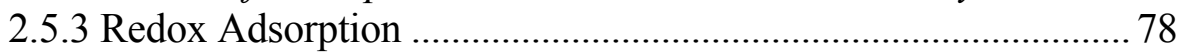

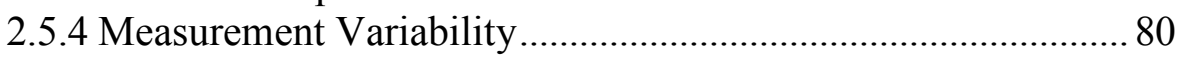

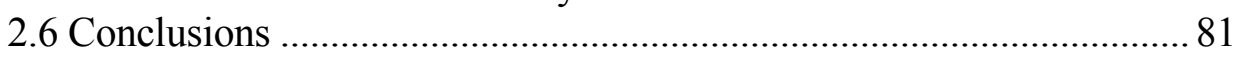

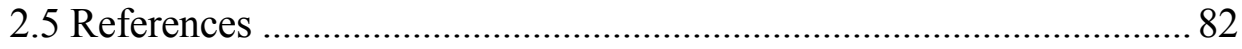


1.1 Modulation of the Conductance of a Semiconductor .8

1.2 Schematic Representations of Surface States .......................................... 9

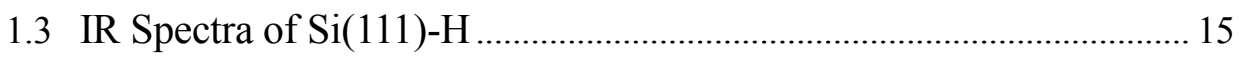

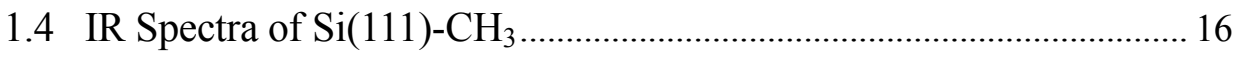

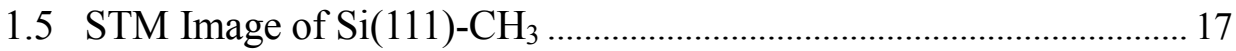

1.6 Schematic Illustration of STS Tip-Induced Band Bending ................. 18

1.7 SXPS C 1s and Si 2p Spectra of $\mathrm{Si}(111)-\mathrm{CH}_{3}$ and $-\mathrm{C}_{2} \mathrm{H}_{5}$ Surfaces ..... 21

1.8 Energy Level Diagram of Si in an XPS with Band Bending Shift ..... 23

1.9 XPS Si 2p spectra of p-Si(111)-H and $\mathrm{p}^{+}-\mathrm{Si}(111)$-H Surfaces.............. 25

1.10 Angle Resolved XPS Si 2p Spectra of $\mathrm{p}^{+}-\mathrm{Si}(111)-\mathrm{H}$ Surface ............. 26

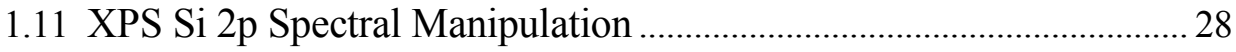

1.12 Energy Level Schematic with Dipole Shifts of a Redox Couple ....... 29

1.13 Open Circuit Voltage vs Effective Solution Potential .......................... 30

1.14 Schematic of Band Bending and Surface Recombination .................... 31

2.1 Schematic Representation of $\operatorname{Si}(111)$ Surface Functionalization ........ 47

2.2 Energy Level Diagram of Si in an XPS Demonstrating Dipole Shift 51

2.3 XPS Survey Spectra of Si(111) Surfaces …………………………........ 55

2.4 XPS Br 3d Spectra of Si(111) Surfaces ................................................. 56

2.5 IR Absorbance Spectra of Si(111) Surfaces .......................................... 57

2.6 IR Absorbance Spectra of Si(111) Surfaces ............................................ 58

2.7 XPS Si 2p Spectra of Moderately Doped Si(111) Surfaces................... 59

2.8 Si 2 $p_{3 / 2}$ Binding Energy of Functionalized Si Surfaces......................... 60

2.9 XPS Si 2p Spectra of Highly Doped Si(111) Surfaces.......................... 62

2.10 Si 2p $p_{3 / 2}$ Binding Energies vs X-ray Power ............................................ 65 


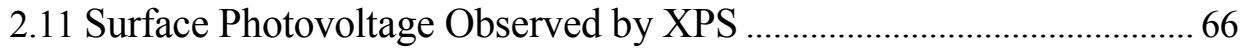

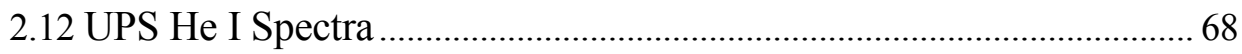

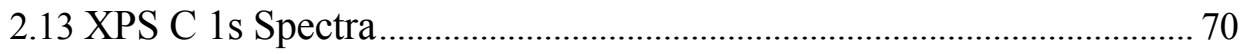

2.14 Band-Level Diagrams of Model System ............................................... 77 


\section{LIST OF TABLES}

Number Page

1.1 Contact Potential of Si Samples after Treatment ................................. 11

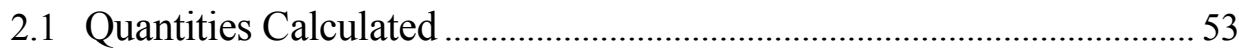

2.2 Si $2 p_{3 / 2}$ Binding Energy of Functionalized Si Surfaces ...................... 61

2.3 Work Function and Surface Dipole of Functionalized Si Surfaces .... 69 


\section{Chapter 1}

\section{Silicon Surfaces and Band Bending}

\subsection{Importance of Semiconductors}

It would be difficult to overstate the role of semiconductors in the massive technological transformations that have occurred in the last half century. Broadly speaking, the two main uses of semiconductors have been in the development of electronic components for computing and in applications for solar energy collection. Looking to the future, a great deal of civilization's hopes rest on continued technological advancement using semiconductors.

Advancements in both electronic components and solar cells require increasing the efficiency of the devices or, to state it a different way, decreasing the energy lost as waste heat. Heat is produced in semiconductors during recombination, in which mobile charge carriers of opposite sign (electrons in the conduction band and holes in the valence band) combine, releasing energy as heat. Silicon, the dominant semiconductor used for electronic devices and solar cells, is an indirect band gap semiconductor, so spontaneous recombination in the bulk of the material is much slower than for other materials. Further, as devices are miniaturized or constructed with cheaper polycrystalline material, recombination at surfaces and interfaces plays an even larger role in limiting the efficiency of the device. Understanding and controlling surface recombination is a major objective of research. This work is an examination of the effects of silicon surface chemistry on the 
surface electronic properties of silicon and an exploration of the use of X-ray photoelectron spectroscopy to measure the band bending and surface dipole of semiconductor samples. These insights and techniques can provide direction for controlling the electronic properties of a surface or interface.

\subsection{Semiconductor Physics}

The versatility and usefulness of semiconductors in a variety of applications arise from the physical and electronic properties of the materials. ${ }^{1-2}$ In crystalline solids, atomic energy levels form band structures with a range of energy states within each band. The frontier orbitals that would form the LUMO and HOMO instead form the valence band and conduction band respectively. The difference between the valence band maximum and the conduction band minimum is the band gap, which for silicon is $1.12 \mathrm{eV}$. If the band gap is very small or nonexistent, as is the case for metals, then electrons in the frontier orbitals are able to gain or lose small amounts of thermal energy in order to occupy a previously unoccupied energy state. If the band gap is very large, as is the case for insulators, electrons in frontier orbitals are effectively locked into their energy state. The band gap of semiconductors are in an electronic "sweet spot" which allows charge carrier populations in both the conduction and valence band to be variable and manipulated.

Electrons can only move into an unoccupied energy state. Electron movement through crystalline solids is analogous to a very simple bucket brigade. In the analogy, atoms are represented by people and electrons are represented by buckets. If no people in a bucket brigade have buckets, then obviously no buckets will move down the line. At the 
other extreme is a situation in which all of the people in the bucket brigade are holding buckets. Assuming no person can hold two buckets, buckets cannot be set down, and people cannot simultaneously give and receive a bucket, then no buckets would be able to move down the line and the situation would be identical to the situation with no buckets. An empty handed person is required to take a bucket and free up another empty handed person in turn. In the most efficient bucket brigade, exactly half of the people would have a bucket at any point in time.

In the semiconductor system, an electron in a mostly empty line of states, the conduction band, is a highly mobile charge carrier. An empty state in a mostly filled line of states, the valence band, is a positively charged mobile carrier called a hole. The context in which the term is used is generally sufficient to distinguish between electrons as mobile charge carriers and electrons in fixed states, such as core levels, throughout the semiconductor. As the number of mobile charge carriers of either type increases, the conductivity of the semiconductor increases as well.

Holes and electrons can be introduced to semiconducting material via dopant atoms, which differ from the atoms they replace by the number of valence electrons present. For silicon and germanium, n-type material will be doped with either phosphorous or arsenic, which both have five valence electrons. The extra electron goes into the conduction band and although it will be electrostatically attracted to the positively charged nucleus of the dopant atom, it will have increased mobility due to the empty conduction band states surrounding it. The p-type material will have excess holes due to boron 
substitution into the lattice structure. The boron with three valence electrons will attract to it an electron from the valence band, creating a positively charged hole and leaving boron negatively charged, thereby maintaining charge neutrality.

Doping of material changes both the conductivity of the material as well as the position of the Fermi level within the band gap. The Fermi level is a hypothetical energy level that has a $50 \%$ probability of being occupied by an electron at thermodynamic equilibrium. For a metal at absolute zero, the Fermi level is the energy level of the highest energy electron. In an electrochemical system, the Fermi level is the chemical potential of the system. In a semiconductor, even at very high dopant levels, an energy level in the valence band will always have greater than $50 \%$ probability of being filled and likewise an energy level in the conduction band will have less than $50 \%$ probability of being filled. Therefore, the Fermi level of a semiconductor, in the absence of an electric field, will be located within the band gap. For intrinsic silicon, the Fermi level will be located half way between the valence band maximum and the conduction band minimum. N-type doping in semiconductors pushes the Fermi level closer to the conduction band as a function of the dopant density. Likewise, p-type dopants push the Fermi level closer to the valence band. When materials with different Fermi levels come into contact, electrons are transferred from the material with the higher energy Fermi level until the Fermi levels are equilibrated. For semiconductors, dopant atoms near the interface become ionized, establishing an electric field which shifts the energy of the valence band and conduction band with respect to the Fermi level in a process called band bending. 
A p-n junction is a good model system for understanding band bending in semiconductors. As electrons flow from the n-type material to the p-type material and holes flow in the opposite direction, they will ionize dopants and establish an electric field until the energy required to move a charge through the electric field is equal to the initial difference between the Fermi levels. The distance from the interface to the furthest ionized dopants, the depletion width, will be a function of the projected charge per unit area and the dopant density. After equilibration, the Fermi level remains flat and the charge neutrality of the system is maintained.

The band bending observed in other systems is not as straightforward. At semiconductor metal contacts, ${ }^{3}$ vacuum contacts, ${ }^{4-5}$ and solution contacts, ${ }^{6-7}$ charged surface states can contribute to the total charge which must be balanced by the charge in the depletion region of the semiconductor. Surface states can be grouped into intrinsic surface states and extrinsic surface states. Intrinsic surface states are ascribed to "clean wellordered" surfaces and will be present on a perfect surface, while extrinsic surface states are present due to a perturbation from the ideal surface. ${ }^{8}$ These perturbations can include defect sites, step edges, and adsorbed species. Intrinsic surface states are the result of the termination of a periodic crystal potential, first described by Igor Tamm and subsequently by William Shockley. ${ }^{9}$ Due to the greater theoretical interest in intrinsic surface states, the term is often shortened to just "surface states" and intrinsic states are often assumed unless explicitly stated otherwise. In this thesis the term is used to indicate both intrinsic and extrinsic surface states. 
Fermi level pinning takes place when the charged surface states dominate the position of the Fermi level at the semiconductor interface. In systems without a contacting material (i.e., in vacuum) band bending must be the result of charged surface states making these systems useful for studying the nature of surface states. In moderately doped systems the density of charged surface states, which would cause band bending spanning the entire band gap, would require only $0.02 \%$ of surface atop sites to have a charge associated with them.

\subsection{History of Semiconductor Device Development and Surface}

\section{States}

The invention of the first transistor was closely tied to basic research on surface states. Semiconductor transistors were intentionally researched and developed to replace the existing technology of mechanical relays and vacuum tubes that were in use in various

electronic applications in the 1940s. ${ }^{10}$ Relays, in which electromagnets controlled by small power inputs would open or close circuits controlling larger power outputs, were constrained by the speed at which the mechanical switch could open and close. Vacuum tubes could also be used as switches and amplifiers but they required considerable power and had short shelf lives due to filament burnout and vacuum leaks. Semiconductors were known to have variable resistance due to temperature and it was understood at the time that if the variable resistance of semiconductors could be controlled electronically rather than thermally, then relays and vacuum tubes could be replaced. 
Development of solid state semiconductor devices to replace relays and vacuum tubes was pursued in earnest at Bell Telephone Laboratories under the direction of William Shockley, with John Bardeen and Walter Brattain working on the project. Initial attempts by Shockley to modulate the conductivity of semiconductors employed a semiconducting material as one plate in a parallel plate capacitor in order to apply a "field effect" to change the resistivity of the semiconductor. As shown in Figure 1.1, a charged metal plate was hypothesized to induce charges in the semiconducting material and therefore increase the conductivity. $^{11}$ This scheme, however, did not work ${ }^{12-13}$ and a model was proposed by John Bardeen to explain the independence of the work function to the position of the Fermi level in the bulk of the semiconductor. ${ }^{14}$ Surface states were proposed to exist at the free surface of the semiconductor and these states could be charged in the absence of an external field or metal contact. If the semiconductor was exposed to an external field or metal contact, these surface states would charge or discharge to compensate and prevent or lessen any change in the charge concentration in the space charge region (Figure 1.2). 

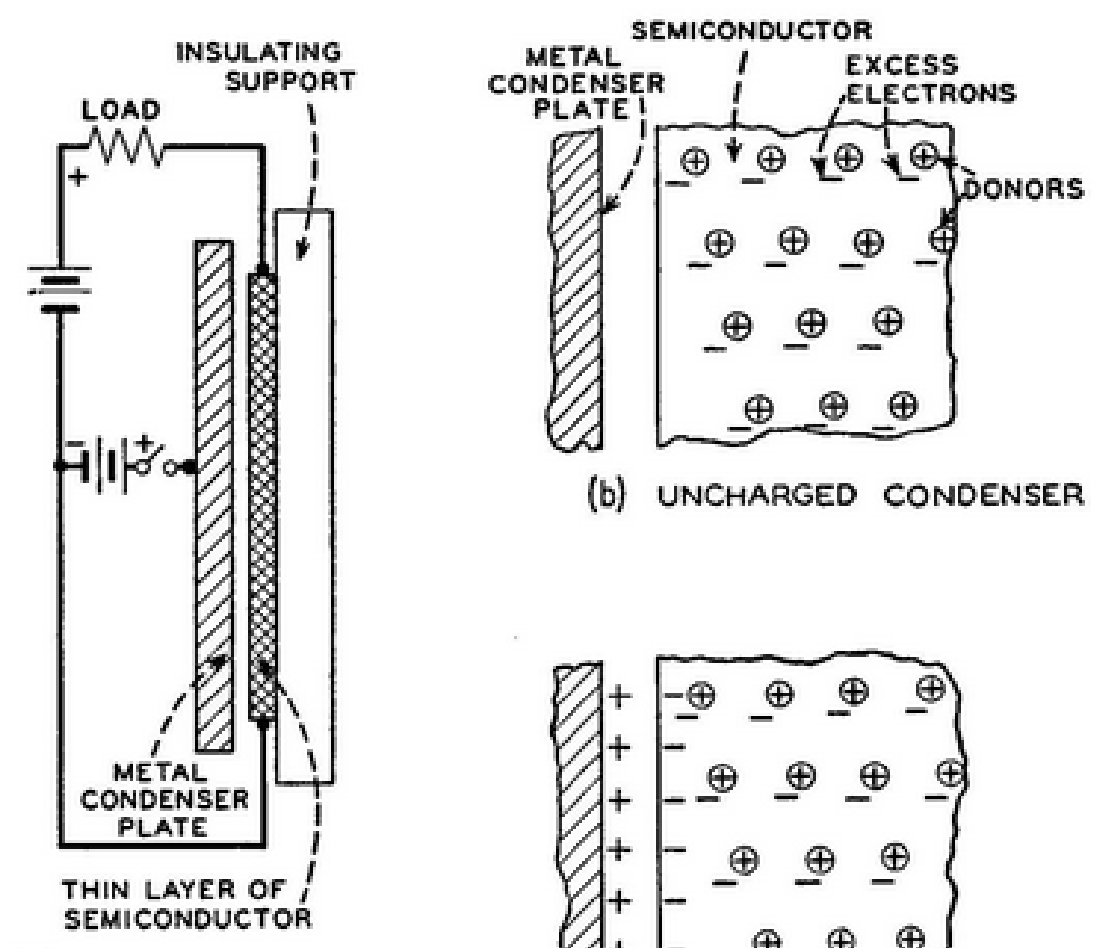

(b) UNCHARGED CONDENSER

(a) PHYSICAL SCHEME

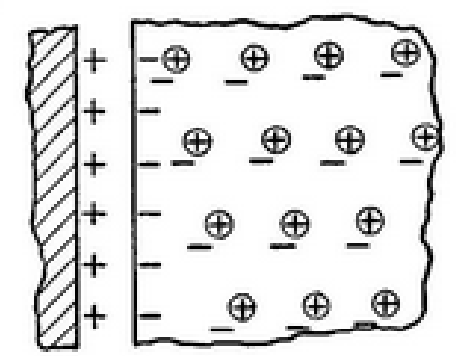

(c) CHARgeo CONDENSER

Figure 1.1 Modulation of the Conductance of a Semiconductor. Schematic representation of the "field effect". 11 
NEUTRAL SEMICONDUCTOR SURFACE

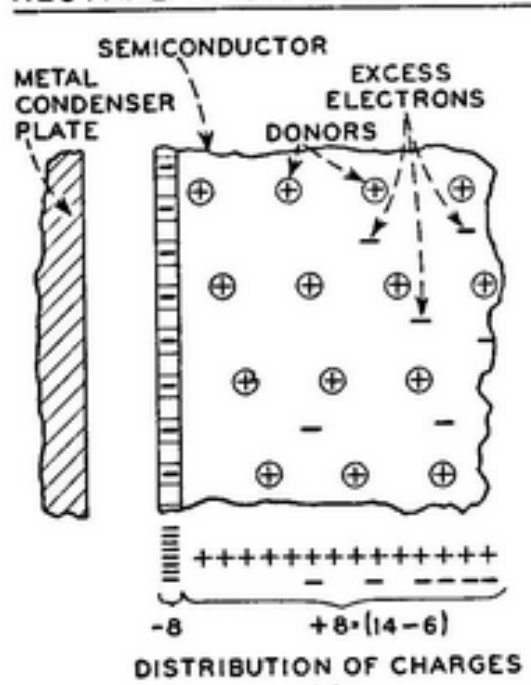

(a)
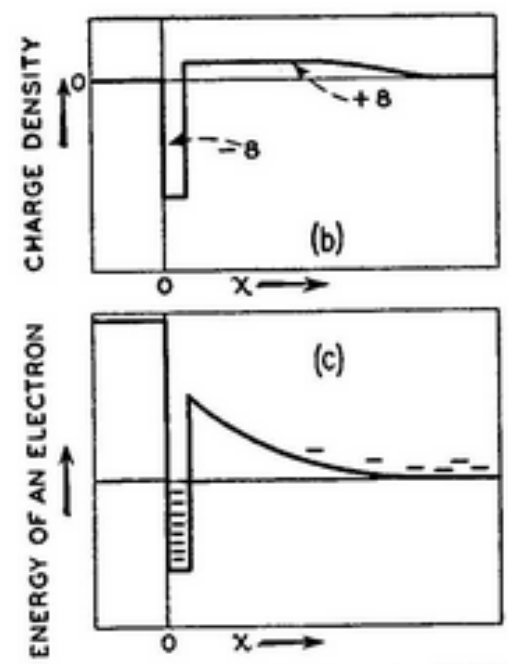

DISTANCE INTO SEMICONDUCTOR
NEGATIVELY CHARGED SURFACE

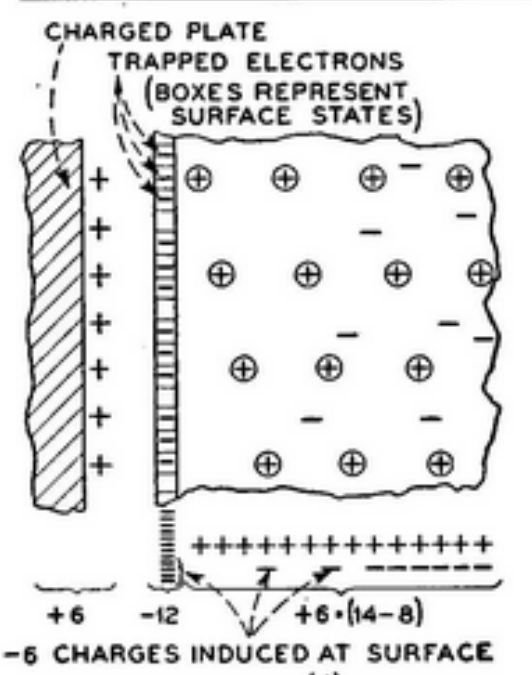

(d)
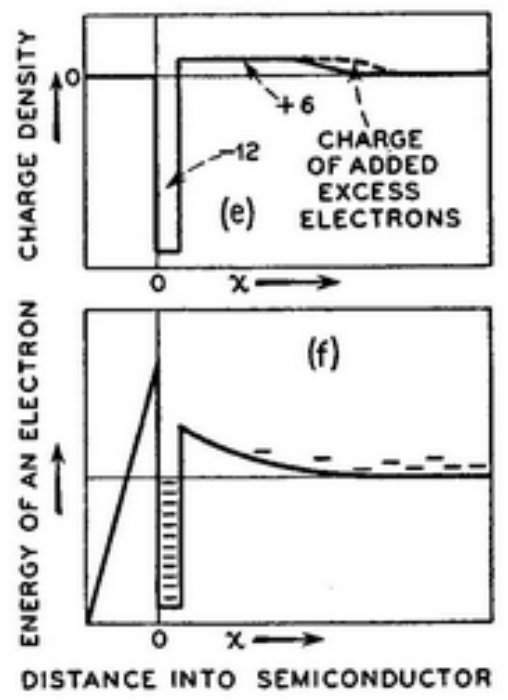

Figure 1.2 Schematic Representations of Surface States. Charged surface states present in the absence of an electric field (a-c) and the compensation of an external charge by additional surface states. ${ }^{11}$

It is unclear what exactly Bardeen thought about the nature of the proposed surface states at the time. There is some evidence that he was ambivalent concerning whether the surface states were intrinsic or extrinsic. In a Bell Labs Memorandum titled "The Genesis of the Transistor," W. Gorton wrote, "These results [Shockley's initial failures] led to a 
reexamination of the theory and the postulation by J. Bardeen of the trapping of electrons

in the surface layers of, or adsorbed layers on, semiconductors, especially silicon and germanium" $" 15$ [emphasis added]. In his landmark paper he explains the theory behind intrinsic surface states and refers to them as Tamm levels, but Bardeen also writes, "Surface states may also result from surface imperfections, from having foreign atoms on the surface, etc."14 His theoretical description of what will become known as Fermi level pinning is identical for both intrinsic and extrinsic surface states.

The decision was made at Bell Labs to continue research on surface states. W. Gorton wrote:

The nature of surfaces and surface phenomena have always posed some of the most difficult problems in solid-state physics. Bardeen's theory afforded a means of investigating the electronic behavior of the surface and of determining the properties and origin of the surface states - results which would constitute important contributions to the science of surface phenomena. There was also the possibility that if action of the surface states could be prevented, then the field effect would become efficient, and electronic semiconductor amplifiers might become practical. Research on the nature of the surface-state effects was thus seen to have the usual combination of advantages of being physical research of fundamental scientific importance, of involving skills, instruments, and materials which were available almost uniquely at Bell Telephone Laboratories, and of having, at the same time, the possibility of leading to developments of great practical importance. The obvious decision was, therefore, made to stress research on the surface states, and as described below, the phenomena which led up to the invention of the transistor were discovered in the course of this fundamental research program. ${ }^{15}$

Clearly the concept of surface states was important to them at the time and crucial to the development of the transistor. 
Brattain demonstrated in July 1947 that silicon surfaces could have variable concentrations of surface states by measuring the contact potential difference between ntype and p-type silicon with a range of dopant densities and platinum. This method of contact potential difference measurement can effectively measure the change in work function across various samples. Initially, all samples had similar contact potentials, but after heat treatment at $400^{\circ} \mathrm{C}$, the p-type samples demonstrated a significant change, with the greatest deviation present in the samples with the highest dopant density as shown in Table 1.1. ${ }^{16}$ This was the first example of band bending of p-type, but not n-type, silicon samples in air, and it was a demonstration that the band bending could be induced by exposure to air. Further, Brattain demonstrated a change in the contact potential difference due to illumination, also known as surface photovoltage (SPV), when samples were cooled to $120 \mathrm{~K} .{ }^{17}$ The absence of SPV at room temperature was likely the result of high recombination rates of samples prepared by grinding and sand-blasting rather than polishing and wet chemical etching as is used today.

Table 1.1: Contact Potential (V) of Si Samples after Stated Treatment ${ }^{16}$

\begin{tabular}{llllll}
\hline $\begin{array}{l}\text { Dopant } \\
\text { Type }\end{array}$ & $\begin{array}{l}\text { Dopant } \\
\text { Density } \\
\left(\mathrm{N} / \mathrm{cm}^{2}\right)\end{array}$ & $\begin{array}{l}\text { In air after } \\
\text { sandblast } \\
(\mathrm{V})\end{array}$ & $\begin{array}{l}\text { In vacuum after } \\
\text { heat treatment } \\
(\mathrm{V})\end{array}$ & $\begin{array}{l}\text { After } \\
\text { exposure } \\
\text { to } \mathrm{N}_{2}\end{array}$ & $\begin{array}{l}\text { After } \\
\text { exposure } \\
\text { to air }\end{array}$ \\
\hline $\mathrm{P}$ & $5.7 \times 10^{20}$ & +0.31 & -0.27 & -0.19 & +0.07 \\
$\mathrm{P}$ & $1.5 \times 10^{20}$ & +0.35 & -0.18 & -0.10 & +0.13 \\
$\mathrm{P}$ & $6.5 \times 10^{18}$ & +0.30 & -0.10 & -0.09 & +0.17 \\
$\mathrm{P}$ & $3.1 \times 10^{17}$ & +0.34 & +0.04 & +0.14 & +0.28 \\
$\mathrm{~N}$ & $6.9 \times 10^{18}$ & +0.32 & +0.16 & +0.27 & +0.34 \\
$\mathrm{~N}$ & $2.3 \times 10^{19}$ & +0.37 & +0.27 & +0.35 & +0.39 \\
$\mathrm{~N}$ & $1.9 \times 10^{20}$ & +0.37 & +0.30 & +0.37 & +0.37 \\
\hline
\end{tabular}


Progress had stalled on implementation of a semiconductor in a variable resistance device until Brattain discovered that he could effectively remove the surface state charge by immersing a silicon electrode into an electrolyte solution. ${ }^{15,18}$ Within one month, the members of the Bell Labs team used the new insights gained to design and build the first transistor ${ }^{10,19}$ by using gold contacts on germanium to directly inject minority carriers. $^{20-21}$ Concurrent with their publication of the first transistor, for which they received the Nobel Prize in Physics, the members of the Bell Labs team published two other letters to the editor in Physical Review detailing their work on semiconductor surfaces. N-type germanium samples were found to have conductivity that varied with surface treatment when the different surfaces were anodized, oxidized, sandblasted or etched. $^{22}$ Shockley meanwhile measured surface state densities on various thin films and obtained similar values from Brattain's previous experiments. ${ }^{12,16}$ Development progressed rapidly for semiconductor electronic devices, with the issue of surface states being effectively bypassed due to direct minority carrier injection.

All three Nobel Laureates from the Bell Labs team briefly returned to the issue of semiconductor surface states in the subsequent years. In 1953 Brattain and Bardeen demonstrated that changes to the contact potential of germanium surfaces could be achieved by changing the gaseous ambient, with ozone/peroxide vapors and water/alcohol vapors having dominant and opposite effects. ${ }^{23}$ In their framework, changes to the contact potential were "assumed to result from adsorption and desorption of fixed ions," and "the charge of the ions is compensated mainly by charges in the surface traps which, together with the ions, form a double layer." Thus the total charge of the surface states was the 
combination of both the adsorbed surface ions and the charging/discharging of recombination traps. Unfortunately, they refer to a surface dipole by which they mean the charge separation between the charged surface states and the charge in the space charge region. Throughout this thesis, "surface dipole" will refer exclusively to the molecular dipole between the two terminal surface atoms.

Shockley also looked at charged surface states once more in 1963 when he characterized the contact potential of oxidized silicon in "wet" and "dry" ambient conditions. He reported that water vapor increased both the concentration and the mobility of ions on the surface. ${ }^{24}$ While the effects of charged surface states have been observed, direct identification has remained elusive due to the low concentration of such states on the surface. Modern surface preparation techniques provide cleaner, more homogeneous surfaces, ideal for studying the nature of surface states.

\subsection{Silicon Surface Chemistry and Analysis}

\subsubsection{Molecular Modification}

Different silicon facets, labeled by their Miller indices, will have differing arrangements of silicon atoms on the surface. The silicon atoms on the unreconstructed $\mathrm{Si}(111)$ surface form a hexagonal close pack configuration with $0.38 \mathrm{~nm}$ spacing between atop sites. $^{25}$ There are no bonds in the plane of the surface, and each silicon atop site can form one bond perpendicular to the surface. Using aqueous etching it is possible to attain high quality hydrogen terminated $\mathrm{Si}(111)$ surfaces with the $\mathrm{Si}-\mathrm{H}$ bond oriented normal to 
the surface, large flat terraces using low miscut wafers, and very low defect densities. ${ }^{26}$ Hydrogen terminated silicon is routinely used as a starting point for further silicon surface chemistry due to the ease of preparation, cleanliness, and ideality of the surface.

Two dominant methods of silicon surface modification are hydrosilylation and Grignard chemistry. ${ }^{27}$ Grignard chemistry involves first the replacement of the hydrogen atom with a halogen, in either solution or the gas phase, followed by reaction with an alkylmagnesium or alkyllithium reagent. ${ }^{28}$ A primary advantage of this technique is the ability to attach small alkyl molecules including a single methyl group. When bulky sterically hindered alkyl groups are added, the surface coverage is lower than the surface coverage obtained with unhindered groups such as methyl, and some of the non-alkylated sites revert to hydrogen termination. ${ }^{29}$ Hydrosilylation generally involves addition of the $\mathrm{Si}-\mathrm{H}$ bond across a terminal vinyl group, and it is typically used to attach long chain alkyls. $^{30}$ Controlling for the size of alkyl group attached, the Grignard technique was found to have lower rates of oxidation when exposed to air and lower surface recombination velocities when compared to the hydosilylation technique. ${ }^{31}$

\subsubsection{Infrared Spectroscopy}

The high quality of the $\mathrm{Si}(111)-\mathrm{CH}_{3}$ surface has been demonstrated by infrared spectroscopy. The starting $\mathrm{Si}(111)-\mathrm{H}$ surface has $\mathrm{Si}-\mathrm{H}$ bonds oriented perpendicular to the plane of the surface, confirmed by the sensitivity of the stretching mode peak, located at $2083 \mathrm{~cm}^{-1}$, to the polarization of the infrared light (Figure 1.3). ${ }^{26}$ There is also an Si-H bending mode that is located at $627 \mathrm{~cm}^{-1} .^{32}$ After reaction with a non-sterically hindered 
alkyl Grignard the Si-H stretch is observed to be completely removed. ${ }^{33}$ For the Si(111)$\mathrm{CH}_{3}$ surface, a peak at $1257 \mathrm{~cm}^{-1}$ polarized perpendicular to the surface (Figure 1.4) has been ascribed to $\mathrm{C}-\mathrm{H}$ symmetrical bending, known as the "umbrella" mode. A C-H rocking motion, also particular to the $\mathrm{Si}(111)-\mathrm{CH}_{3}$ surface, gives rise to a peak at $757 \mathrm{~cm}^{-1}$ polarized parallel to the surface. ${ }^{32}$

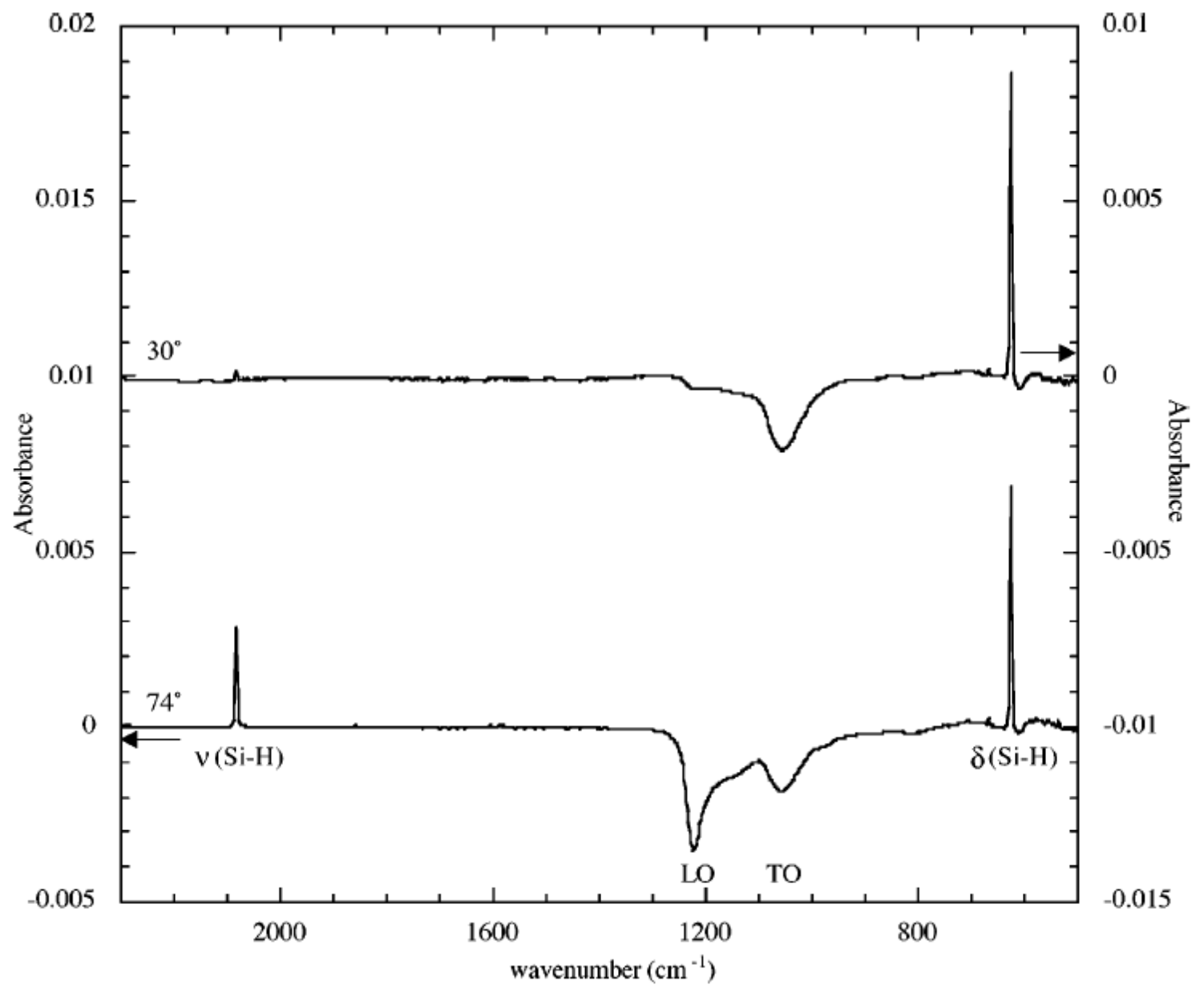

Figure 1.3 IR Spectra of Si(111)-H. The peak at $2083 \mathrm{~cm}^{-1}$ is due to the $\mathrm{Si}-\mathrm{H}$ stretching mode. The absence of peak in the $30^{\circ}$ orientation spectra indicates that the $\mathrm{Si}-\mathrm{H}$ bond is oriented perpendicular to the surface. ${ }^{26,32}$ 


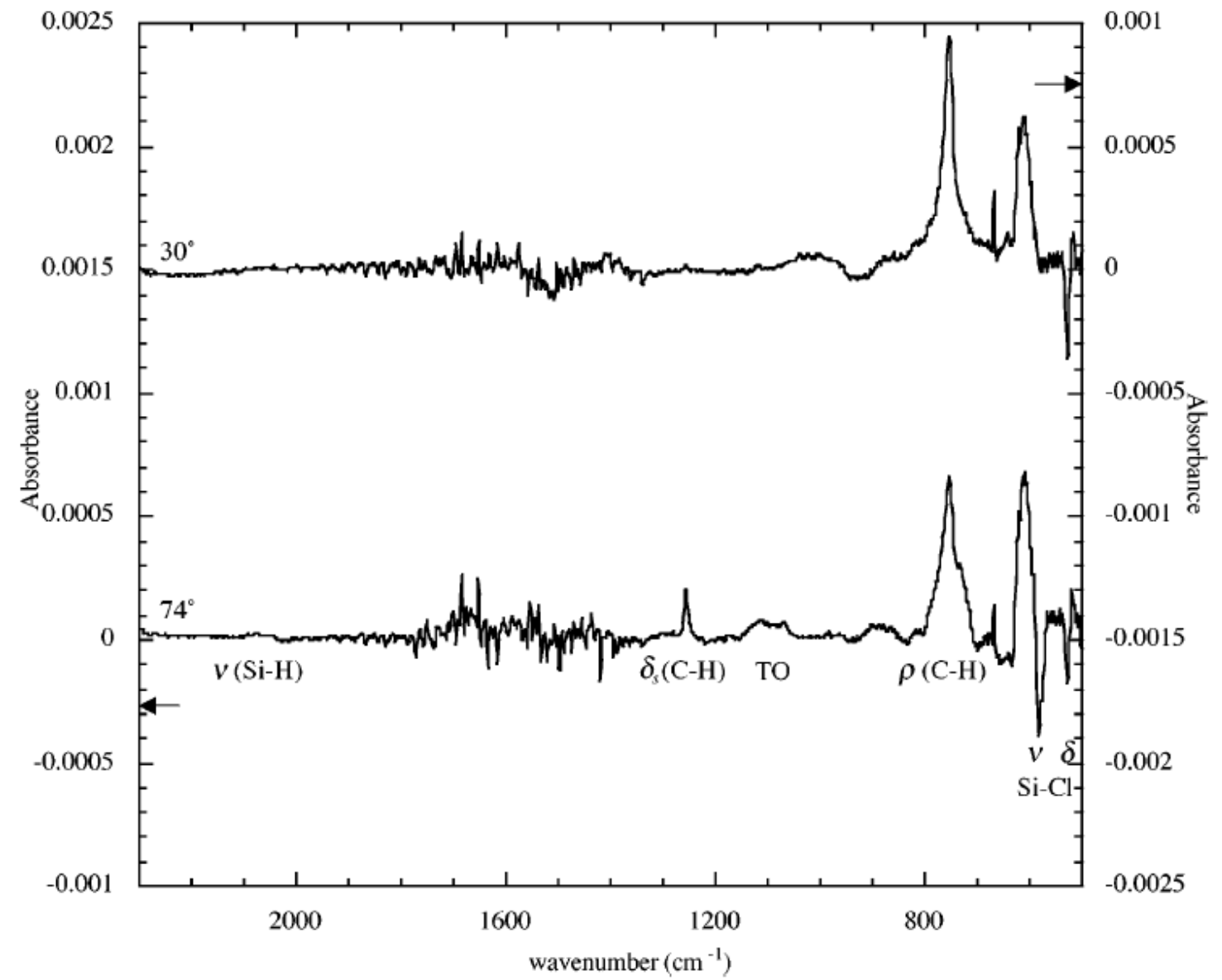

Figure 1.4 IR Spectra of $\mathrm{Si}(\mathbf{1 1 1})-\mathbf{C H}_{3}$. The peak at $1257 \mathrm{~cm}^{-1}$ is due to the $\mathrm{C}-\mathrm{H}$ symmetrical bending. The absence of the peak in the $30^{\circ}$ orientation spectra indicates that the $\mathrm{Si}-\mathrm{C}$ bond is oriented perpendicular to the surface. ${ }^{32}$

\subsubsection{Scanning Tunneling Microscopy}

$\mathrm{Si}(111)-\mathrm{CH}_{3}$ surfaces have been imaged using low-temperature scanning tunneling microscopy (STM) as shown in Figure 1.5. ${ }^{25}$ The surface was found to be highly ordered with few structural defects and complete surface coverage on flat terrace locations. Images collected at $77 \mathrm{~K}$ (Figure 1.5 inset) illustrate the lattice spacing of silicon atop sites on the Si(111) surface with hexagonal close packed spots spaced $0.38 \mathrm{~nm}$ apart. At this temperature the methyl groups spin rapidly on the surface and the image shows the methyl 
groups as a single spot in registry with the underlying atop silicon atoms. Upon cooling

to $4.7 \mathrm{~K}$, the methyl atoms stop spinning on the surface and are frozen into a two dimensional crystal. The individual hydrogen atoms on the methyl group can be resolved with the expected $0.18 \mathrm{~nm}$ spacing between them. Analysis of STM images of Si(111)$\mathrm{C}_{2} \mathrm{H}_{5}$ surfaces indicate that $\mathrm{Si}-\mathrm{C}$ bonds only form at $\sim 80 \%$ of total atop silicon sites. ${ }^{34}$

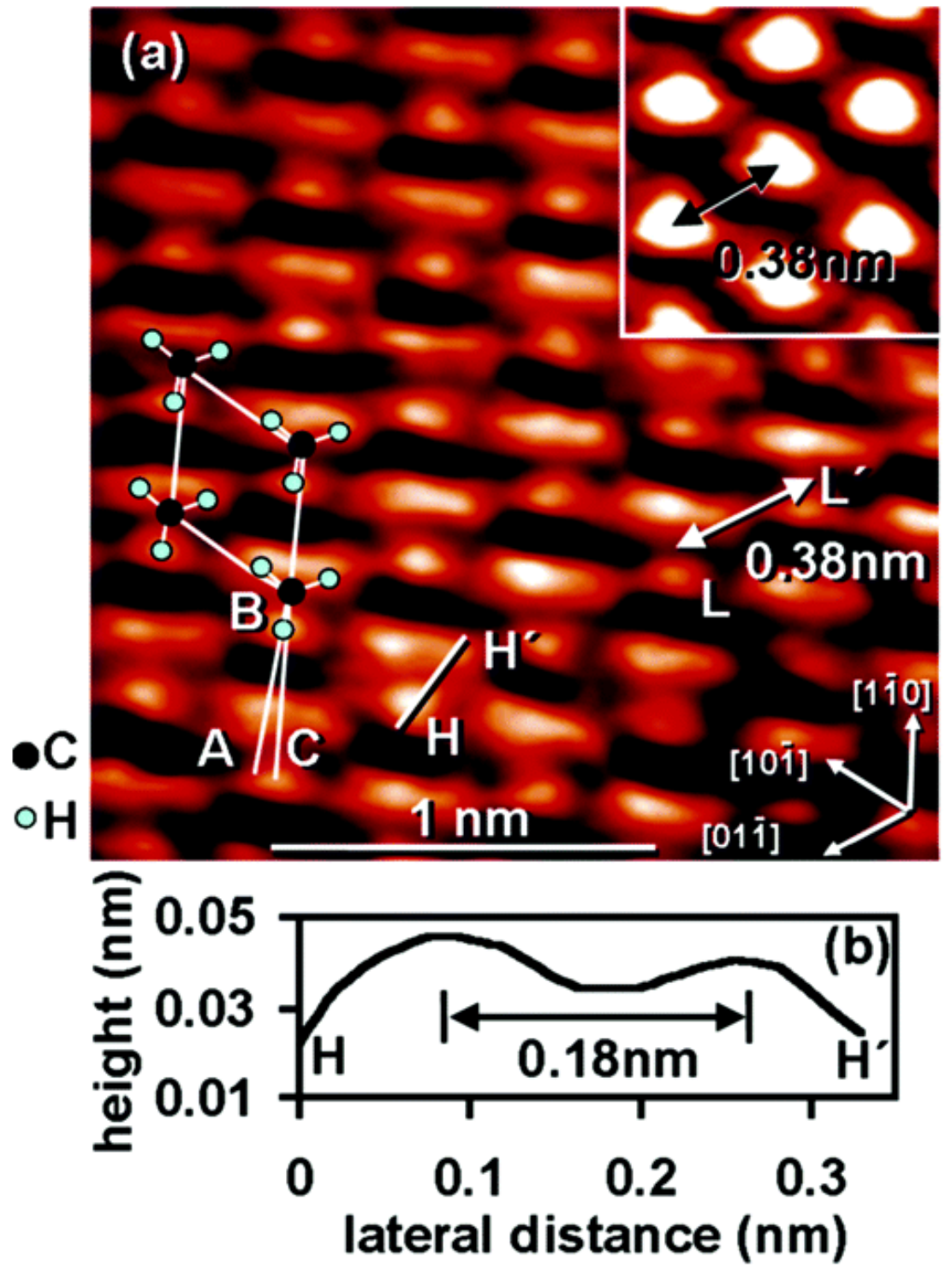

Figure 1.5 STM Image of Si(111)-CH3. STM images taken at $77 \mathrm{~K}$ (inset) and $4.7 \mathrm{~K} .4 .7$ $\mathrm{K}$ image shows resolution of hydrogen atoms on the methyl groups. ${ }^{25}$ 
Scanning tunneling spectroscopy (STS) of hydrogen, methyl, and ethyl terminated $\mathrm{Si}(111)$ surfaces resulted in a conductance gap of approximately $2 \mathrm{~V}$ for all surface types. ${ }^{35}$ The results indicate that there are no observable conductive midgap states and that there is some evidence for tip-induced band bending. Tip-induced band bending has been demonstrated on surfaces with a relatively lower density of surface states (Figure 1.6), even though these surfaces can have "pinned" Fermi levels and/or band bending in the absence of an electric field. ${ }^{36}$

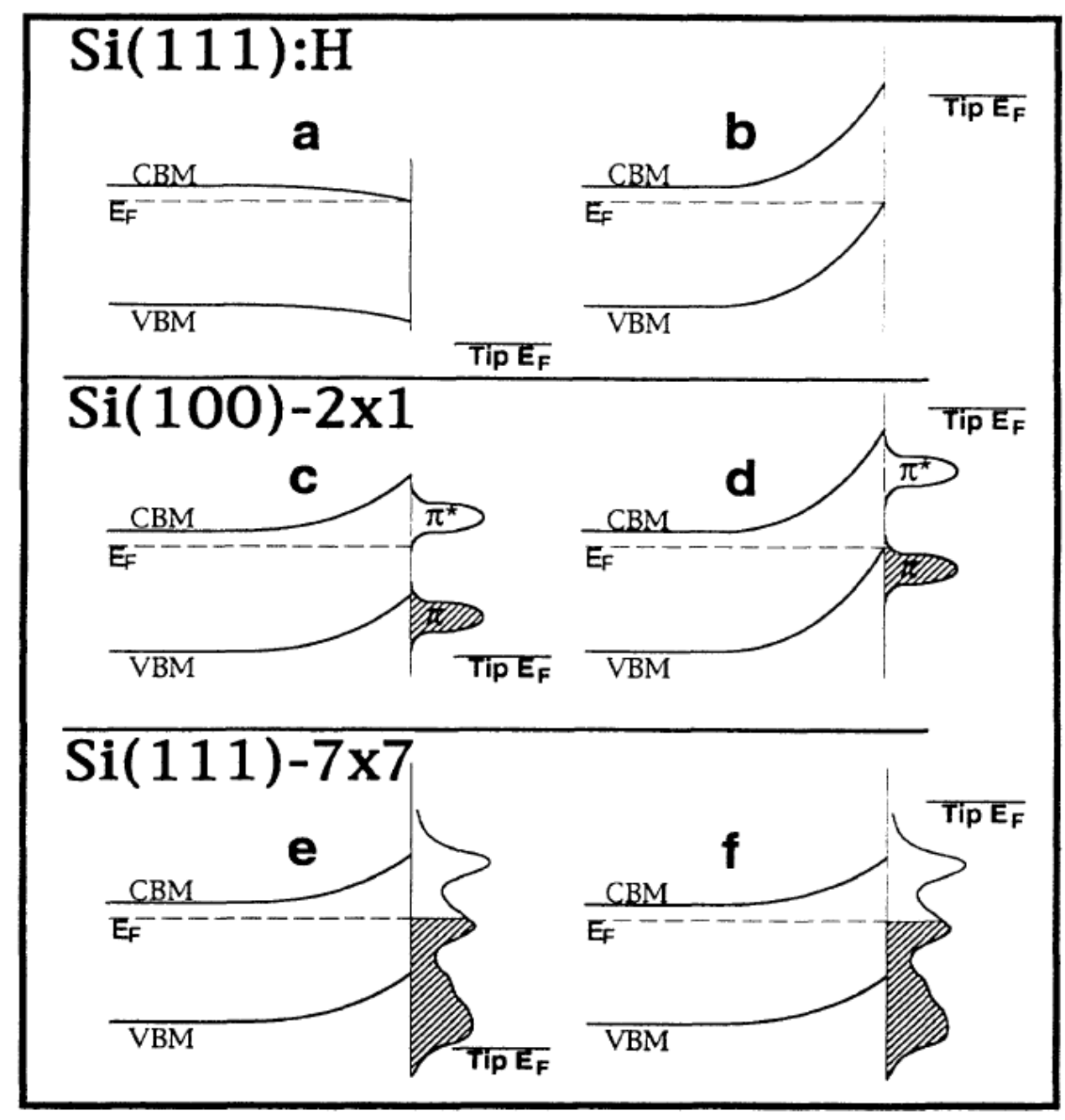

Figure 1.6 Schematic Illustration of STS Tip-Induced Band Bending. Tip-induced band bending observed for $\mathrm{Si}(111)-\mathrm{H}$ surface but not the $\mathrm{Si}(111)-7 \times 7$ surface. $^{36}$ 


\subsubsection{X-ray Photoelectron Spectroscopy}

X-ray Photoelectron Spectroscopy (XPS) has also been used to verify the chemical functionalization of the $\mathrm{Si}(111)$ surface and the near ideal passivation obtained when using a methyl Grignard. The photoelectric effect, the explanation of which won Einstein his Nobel Prize, describes the ejection of electrons from matter due to the absorption of high energy photons. All of the energy from a photon, which can be finely controlled by selecting for a specific wavelength of light using a monochromator, is transferred to an electron. Some of this energy goes toward breaking the connection between the electron and nucleus (this "connection energy" is the sum of the binding energy and the work function of the sample) and the rest of the energy is transferred to the electron as kinetic energy. Any collisions of the emitted electron will reduce the kinetic energy, so XPS measurements are performed in ultrahigh vacuum (UHV) to maximize the number of electrons getting to the detector without losing any kinetic energy. Collisions within the solid sample material will also reduce the kinetic energy, with a $1387 \mathrm{eV}$ electron having

an escape depth through crystalline silicon in the range ${ }^{37}$ of $1.6 \mathrm{~nm}^{38}$ to over $3.9 \mathrm{~nm},{ }^{39}$ making the technique incredibly surface sensitive. Synchrotron sources are able to be even more surface sensitive by causing emission of $150 \mathrm{eV}$ electrons. ${ }^{40}$

The binding energy of an electron is characteristic of the elemental core energy level from which it was emitted, so XPS is a useful tool for determining the elemental composition of a surface. Neighboring atoms with different electronegativity values will affect the charge density on an atom, with electrons from slightly positive atoms being shifted to higher binding energies. For this reason, electrons from silicon or carbon atoms 
bound to oxygen will have a higher binding energy than electrons from pure silicon or pure carbon samples. Each additional bond to oxygen shifts the binding energy of the silicon electron higher, making XPS ideally suited for studying oxidation rates on silicon. $\mathrm{Si}(111)-\mathrm{CH}_{3}$ has been demonstrated to have a slower rate of oxidation in air than $\mathrm{Si}(111)$ $\mathrm{Cl}^{41} \mathrm{Si}(111)-\mathrm{C}_{2} \mathrm{H}_{5},{ }^{41} \mathrm{Si}(111)-\mathrm{H}^{31}$ and samples prepared via hydrosilylation. ${ }^{31}$

XPS is also used to distinguish between carbon bound to carbon and carbon bound to silicon on the surface. The lower electronegativity value of silicon and hydrogen compared to carbon, with Pauling electronegativity values of $1.90,2.20$, and 2.55 respectively, enable XPS to distinguish between $\mathrm{Si}-\mathrm{C}$ bonds and $\mathrm{C}-\mathrm{C}$ bonds on the surface. The signal from $\mathrm{C}-\mathrm{C}$ bonded $\mathrm{C}$ 1s emission is shifted $1.2 \mathrm{eV}$ to a higher binding energy relative to the Si-C C $1 \mathrm{~s}$ emission. ${ }^{42}$ The $\mathrm{C}-\mathrm{C}$ signal attributed to adsorbed adventitious carbon can be removed, indicating desorption, on $\mathrm{Si}(111)-\mathrm{CH}_{3}$ surfaces at $440{ }^{\circ} \mathrm{C}$ as shown in Figure $1.7^{40}$

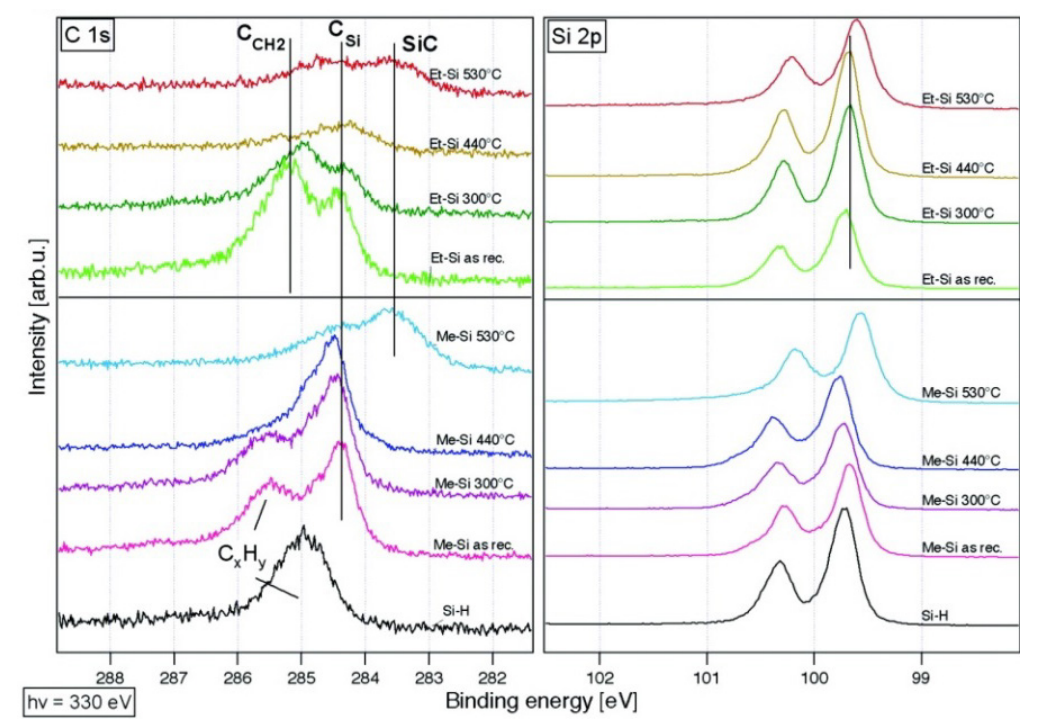

Figure 1.7 SoftXPS C 1s and Si 2p Spectra of $\mathrm{Si}(111)-\mathrm{CH}_{3}$ and $\mathrm{Si}(111)-\mathrm{C}_{2} \mathrm{H}_{5}$ Surfaces. Carbon bound to silicon is $1.2 \mathrm{eV}$ lower than carbon bound to carbon, and the adventitious carbon can be removed on the $\mathrm{Si}(111)-\mathrm{CH}_{3}$ surface by annealing. ${ }^{40}$ 


\subsection{Band Bending Determination by XPS}

Band bending and, more generally, the position of the Fermi level within the band gap can be determined by XPS due to a fortunate quirk in the operation of the instrument. As described above, the energy of the photon $(h v)$ is completely transferred to the electron in order to break the connection to the atom (composed of the binding energy $\left(\mathrm{BE}_{\mathrm{i}}\right)$ and the work function of the sample $\left.\left(W_{\mathrm{F}, \mathrm{i}}\right)\right)$ and to impart kinetic energy $\left(\mathrm{KE}_{\mathrm{i}}\right)$, which can be written as

$$
h v=\mathrm{BE}_{\mathrm{i}}+W_{\mathrm{F}, \mathrm{i}}+\mathrm{KE}_{\mathrm{i}},
$$

where $\mathrm{BE}_{\mathrm{i}}$ is the difference in energy from the core level to the Fermi level, $W_{\mathrm{F}, \mathrm{i}}$ is the difference in energy from the Fermi level to the vacuum level, and the subscript "i" indicates that it is located at the position of excitation. During band bending, all semiconductor energy levels (core levels, valence band, conduction band, and vacuum level) bend in unison relative to the Fermi level. Therefore, the difference in energy from the core level to the vacuum level, the sum of $\mathrm{BE}_{\mathrm{i}}$ and $W_{\mathrm{F}, \mathrm{i}}$, is a fixed value regardless of band bending and $\mathrm{KE}_{\mathrm{i}}$ is unaffected by band bending. However, the kinetic energy is measured at the detector $\left(\mathrm{KE}_{\mathrm{d}}\right)$, and $\mathrm{KE}_{\mathrm{d}}$ is affected by the band bending.

In order to get emitted electrons from the sample to the detector, a small accelerating potential is established that is equal to the difference between $W_{\mathrm{F}, \mathrm{i}}$ and the work function of the detector $\left(W_{\mathrm{F}, \mathrm{d}}\right)$ such that

$$
\mathrm{KE}_{\mathrm{d}}=\mathrm{KE}_{\mathrm{i}}+W_{\mathrm{F}, \mathrm{i}}-W_{\mathrm{F}, \mathrm{d}} .
$$

Substitution into equation 1.1 yields 


$$
\mathrm{BE}_{\mathrm{i}}=h v-\left(\mathrm{KE}_{\mathrm{d}}+W_{\mathrm{F}, \mathrm{d}}\right) .
$$

The values for $h v$ and $W_{\mathrm{F}, \mathrm{d}}$ are properties of the instrument and held constant. The instrument directly measures $\mathrm{KE}_{\mathrm{d}}$, which is only affected by $\mathrm{BE}$, so the XPS measurement will be affected by the band bending and the position of the Fermi level within the band gap. Figure 1.8 demonstrates the effect of band bending on the energy levels and values pertinent to XPS measurements. Values changed by the introduction of band bending are shown in red. All energy levels on the vacuum side of the band bending, in this case a C 1s signal due to adventitious carbon, will also be shifted in energy as a result of the band bending.

Figure 1.8 Energy Level Diagram of Silicon in an XPS Demonstrating Band Bending Shift. Energy level diagram of p-type silicon in an XPS shown (a) without and (b) with band bending, $\mathbf{E}_{\mathrm{bb}}$. The red or dark gray marking in (b) indicates energy levels or values that are affected by $\mathbf{E}_{\mathrm{b} b}$, while the previous locations for the energy levels are depicted in light gray. $W_{\mathrm{F}, \mathrm{s}}$ and $W_{\mathrm{F}, \mathrm{d}}$ are the work functions of the $\mathrm{Si}$ sample and of the detector, respectively, $h v$ is the $\mathrm{X}$-ray energy. $\mathrm{BE}\left(\mathrm{Si} 2 \mathrm{p}_{3 / 2}\right)$ and $\mathrm{BE}(\mathrm{C} 1 \mathrm{~s})$ are binding energies of the Si $2 p_{3 / 2}$ and $\mathrm{C} 1 \mathrm{~s}$ core levels, respectively. $\mathrm{BE}^{\mathrm{V}}\left(\mathrm{Si} 2 \mathrm{p}_{3 / 2}\right)$ is the energy difference between the Si $2 \mathrm{p}_{3 / 2}$ core level and the valence-band maximum. $\quad \mathbf{E}_{\mathrm{vbm}}^{\mathrm{B}}$ and $\mathbf{E}_{\mathrm{vbm}}^{\mathrm{s}}$ are the energies of the Fermi level with respect to the valence-band maximum in the bulk and at the surface, respectively. The arrow for $\mathbf{E}_{\mathrm{bb}}$ is pointing down because the value of $\mathbf{E}_{\mathrm{bb}}$ is negative as drawn. $\mathrm{KE}_{\mathrm{s}}$ is the kinetic energy of the photoelectrons emitted from the indicated core levels at the surface of the sample. $\mathrm{KE}_{\mathrm{d}}$ is the kinetic energy of the photoelectrons as measured at the detector. The valence (VB) and conduction (CB) bands of silicon are shown. Note that the band bending, $\mathbf{E}_{\mathrm{bb}}$, will shift the $\mathrm{Si} 2 \mathrm{p}_{3 / 2}$ and the $\mathrm{C}$ 1s binding energies by the same amount. 
Figure 1.8 Energy Level Diagram of Silicon in an XPS Demonstrating Dipole Shift
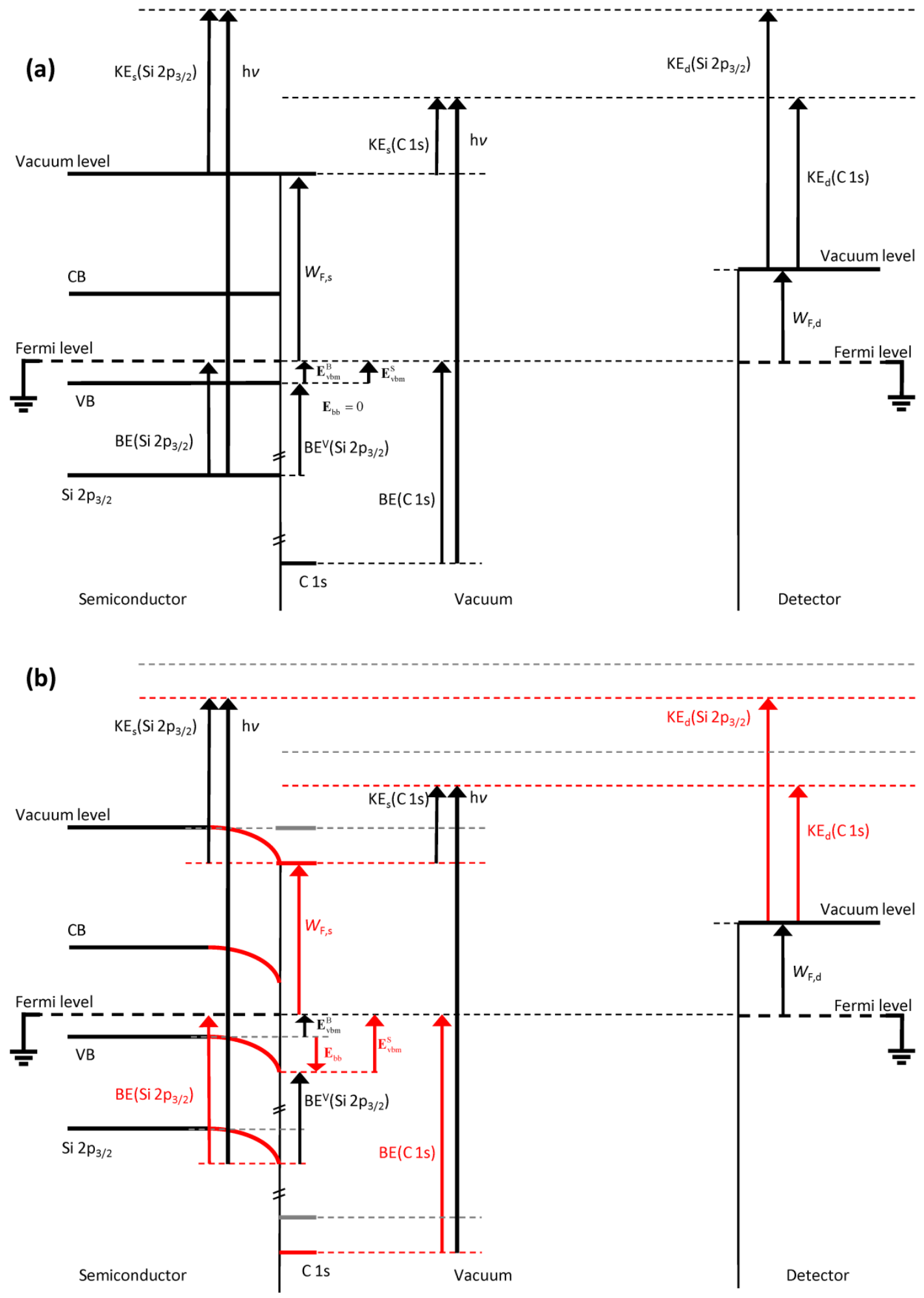
Binding energy shifts due to band bending have been demonstrated previously by XPS. $^{5}$ For moderately doped silicon the escape depth of the photoemitted electrons is much shorter than the depletion width, so even though the band bending is steepest at the surface, all of the detected electrons for a specific peak will come from a narrow range of energies and be shifted by the band-bending energy $\mathbf{E}_{\mathrm{bb}}$. Conversely, if the material is highly doped and the depletion region is on the order of the escape depth of the electron, the detected photoelectrons will have a range of energies corresponding to $\mathbf{E}_{\mathrm{bb}}$ and the peaks will be broadened out. The bottom trace in each spectrum of Figure 1.9 demonstrates the different presentation of band bending for moderately doped (top) and highly doped (bottom) silicon samples. ${ }^{43}$ By rotating the sample in XPS and changing the emission angle, the effective escape depth can be varied, allowing progressively more surface sensitive measurements by blocking out more of the bulk signal. When highly doped samples with large amounts of band bending are analyzed in this way, the binding energy is shifted by the band bending to a greater degree for the more surface sensitive measurements as shown in Figure 1.10. 


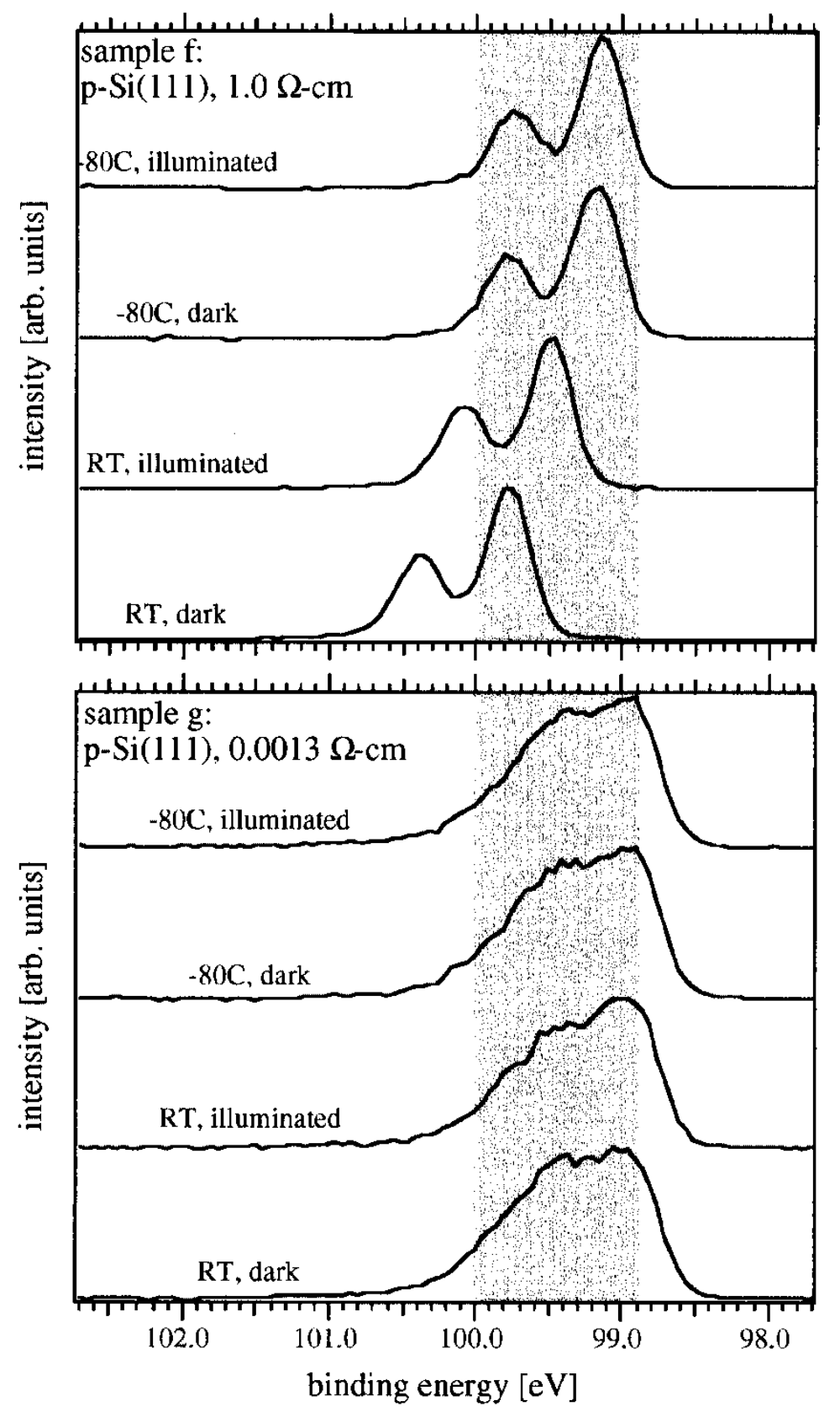

Figure 1.9 XPS Si 2p spectra of p-Si(111)-H and $\mathbf{p}^{+}-\mathrm{Si}(111)-\mathrm{H}$ Surfaces. Si $2 p$ core-level spectra demonstrate band bending in the dark and SPV for low doped sample upon illumination. ${ }^{43}$ 

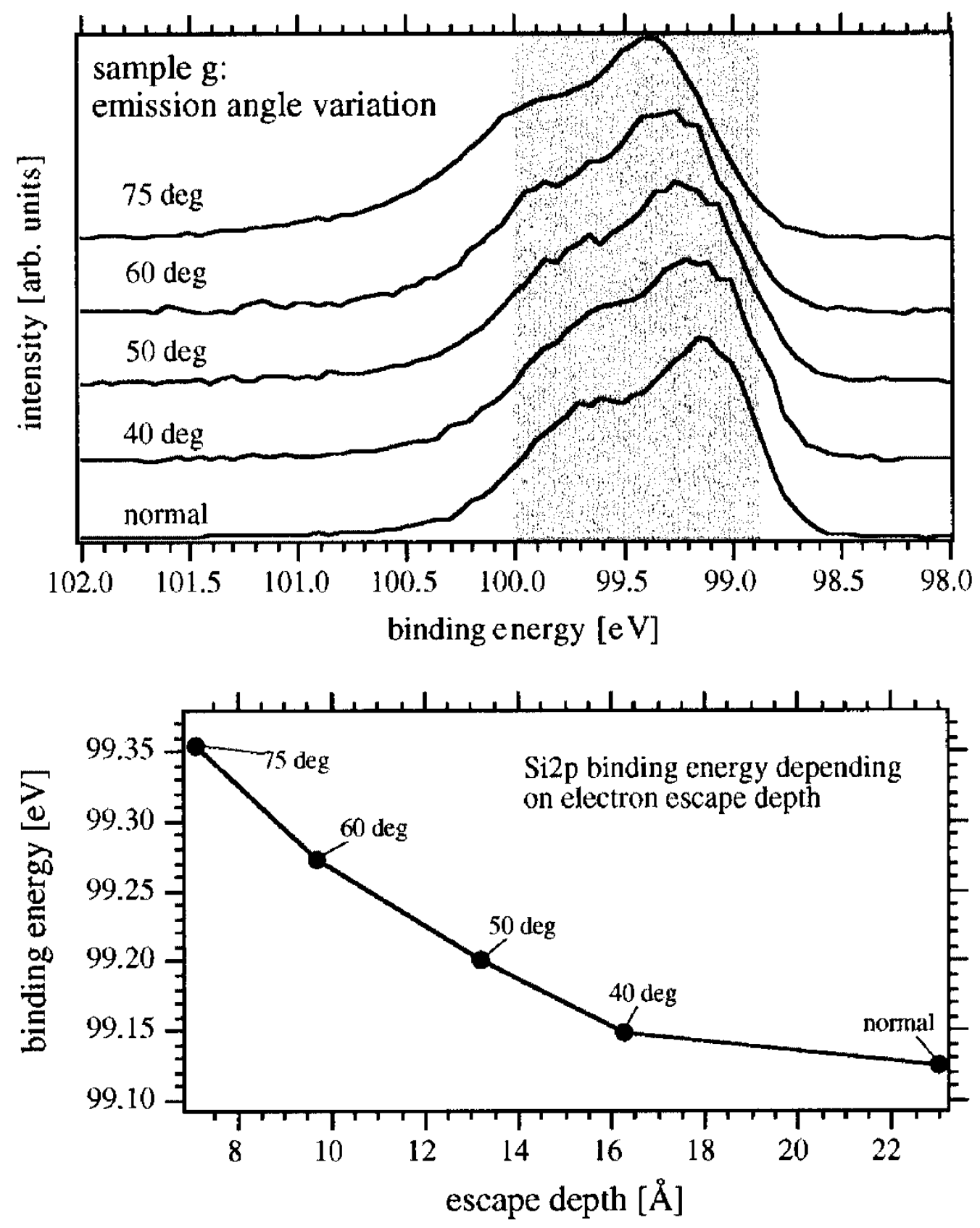

Figure 1.10 Angle Resolved XPS Si 2p Spectra of p $^{+}-$Si(111)-H Surface. Angle resolved spectra demonstrate band bending by shifting away from the bulk binding energy as the spectra get more surface sensitive. ${ }^{43}$ 
In addition to measuring the band bending of semiconductors, XPS can be used to measure the photoinduced flattening of the bands called surface photovoltage (SPV). Photons, with energy larger than the band gap, will generate electrons and holes in the semiconductor. Any charge carriers in the depletion region will be affected by the electric field. Minority carriers will move to the surface and majority carriers will move into the bulk. As minority carriers move to the surface they will either recombine with the charged surface states present or screen some of the charge. In either case the effective charged surface state density will be lowered. In the top spectrum of Figure 1.9, the Si $2 \mathrm{p}$ peak is shifted closer to the expected flat band position upon illumination. Furthermore, when the sample is cooled to $-80{ }^{\circ} \mathrm{C}$, effectively freezing out recombination at the surface, the peak is shifted even closer to flat band conditions. ${ }^{43}$ At the lower temperature, the carrier excitation from the X-ray source alone is enough to flatten the bands to some degree.

A clever proof of concept experiment demonstrating the SPV on silicon involved taking spectra of p-type and n-type silicon simultaneously (Figure 1.11). ${ }^{44}$ In the dark, the binding energy peaks of both materials were fairly close due to Fermi level pinning, but after illumination the peaks from the two different materials shifted apart closer to their expected flat band positions. 


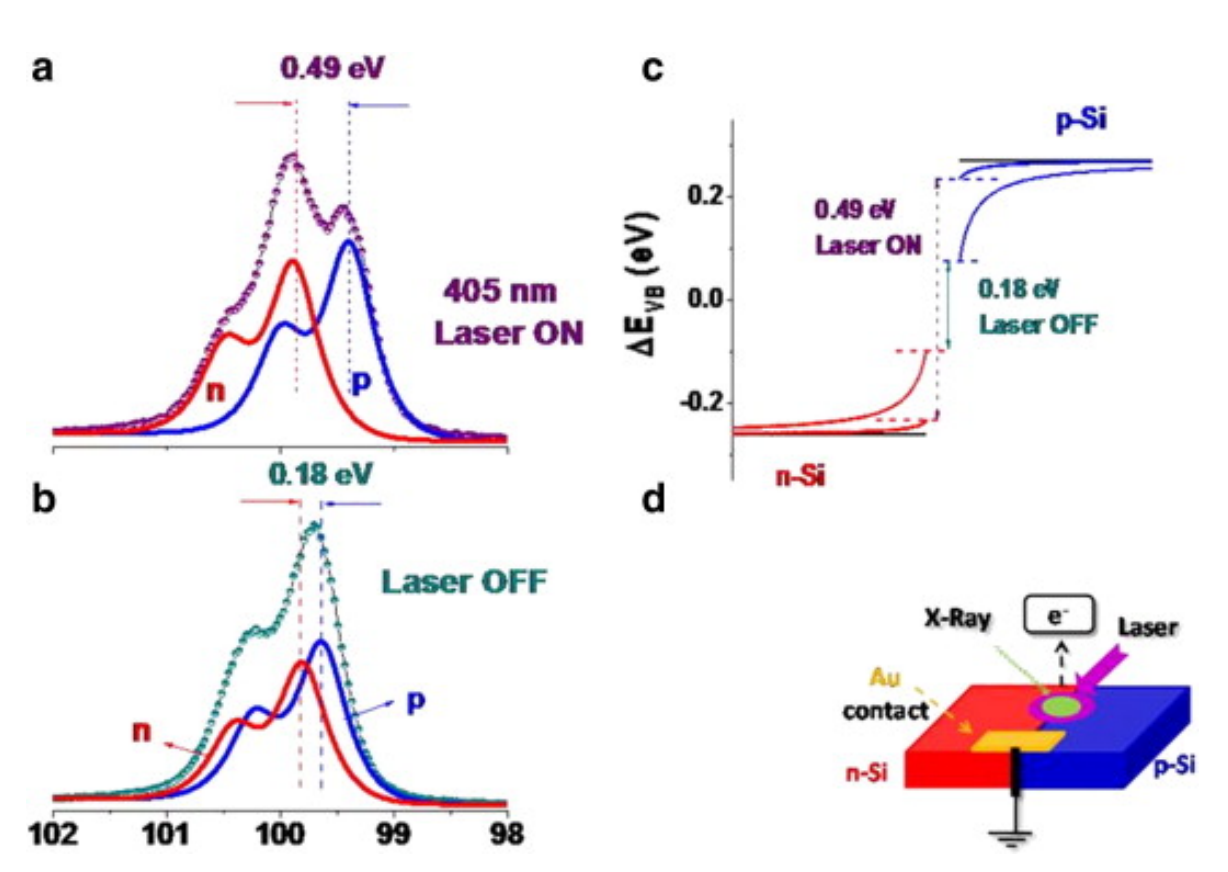

Figure 1.11 XPS Si 2p Spectral Manipulation. Simultaneous Si 2p spectra of n-Si(111)$\mathrm{H}$ and $\mathrm{p}-\mathrm{Si}(111)-\mathrm{H}$ under illumination (a) and in the dark (b) demonstrating that illumination affects the samples differently. ${ }^{44}$

\subsection{Electrochemistry, Band Bending, and Surface States}

In an ideal semiconductor electrochemical system, the Fermi level of the semiconductor will equilibrate with the redox energy level in solution. While early work on semiconductor electrochemistry found evidence for surface states giving rise to Fermi level pinning which limited the potential of the system and reduced the overall efficiency, ${ }^{6-}$ 7 later reports found that surface states could be avoided in some electrochemical systems. $^{45-46}$ A much higher density of surface states is required to pin the Fermi level of a semiconductor electrode compared to a semiconductor in vacuum. ${ }^{7,47}$

Open circuit photovoltage is a measure of dark band bending in a semiconductor photoelectrochemical system. Charge transfer between the redox couple and 
semiconductor can bend the bands until the Fermi level intersects the valence band or conduction band, depending on the redox couple. Upon illumination, the bands will flatten producing a photovoltage. Surface dipoles have been demonstrated to shift the open circuit photovoltages of $\mathrm{Si}(111)$ electrodes in contact with one-electron, outer-sphere redox couples. $^{48}$ A surface dipole will shift the energy level of the redox couple with respect to the valence bands and conduction bands as shown in Figure 1.12. ${ }^{49}$ The $\mathrm{Si}(111)-\mathrm{CH}_{3}$ surface being a negative dipole will push redox couples to a lower energy, or higher redox potential (the sign conventions for energy and potential are flipped). Practically, this means that a $\mathrm{Si}(111)-\mathrm{CH}_{3}$ surface in contact with a redox couple will have the same electrochemical behavior as a $\mathrm{Si}(111)-\mathrm{H}$ surface in contact with a different redox couple with a $0.4 \mathrm{~V}$ greater effective solution potential (Figure 1.13). ${ }^{48}$
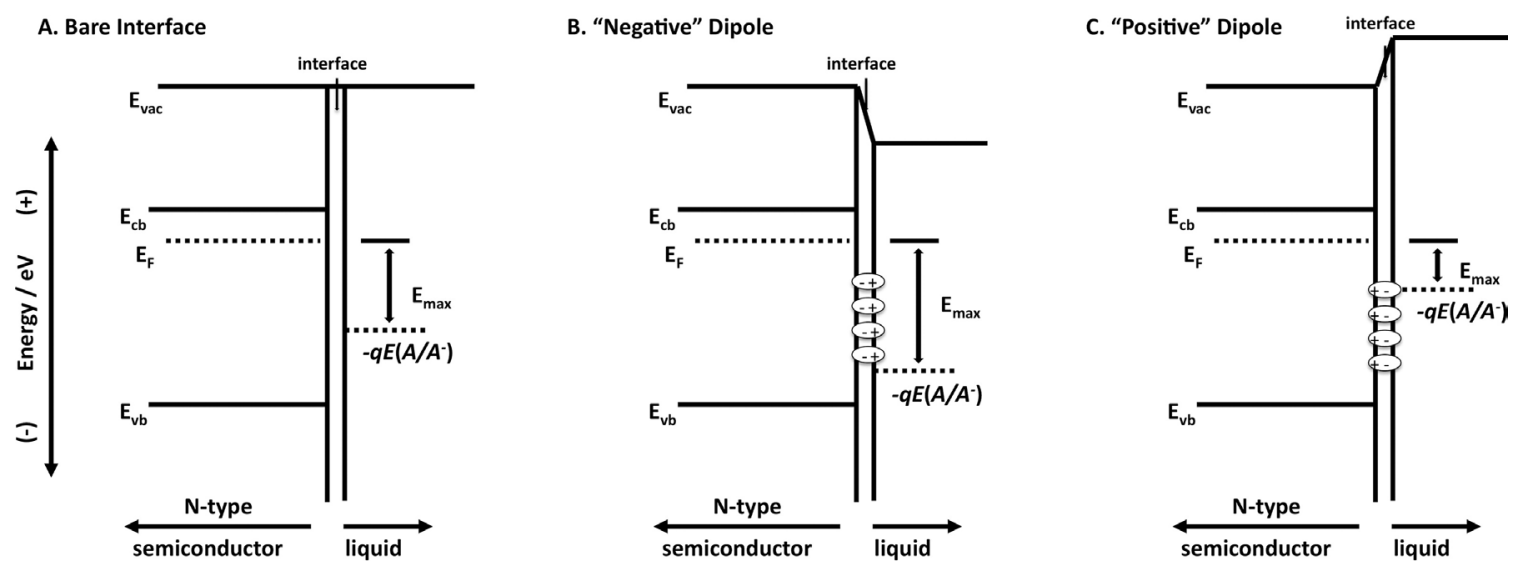

Figure 1.12 Energy Level Schematic Illustrating Dipole Shifts of a Redox Couple. Upon introduction of a negative dipole (b) the energy level of the redox couple is shifted down relative to the valence band and conduction band. A positive dipole (c) shifts the energy level of the redox couple up. Equilibration between the Fermi level and the redox couple will result in band bending for all cases, but (b) will have the largest band bending after equilibration due to the shifted position of the redox couple. ${ }^{49}$ 


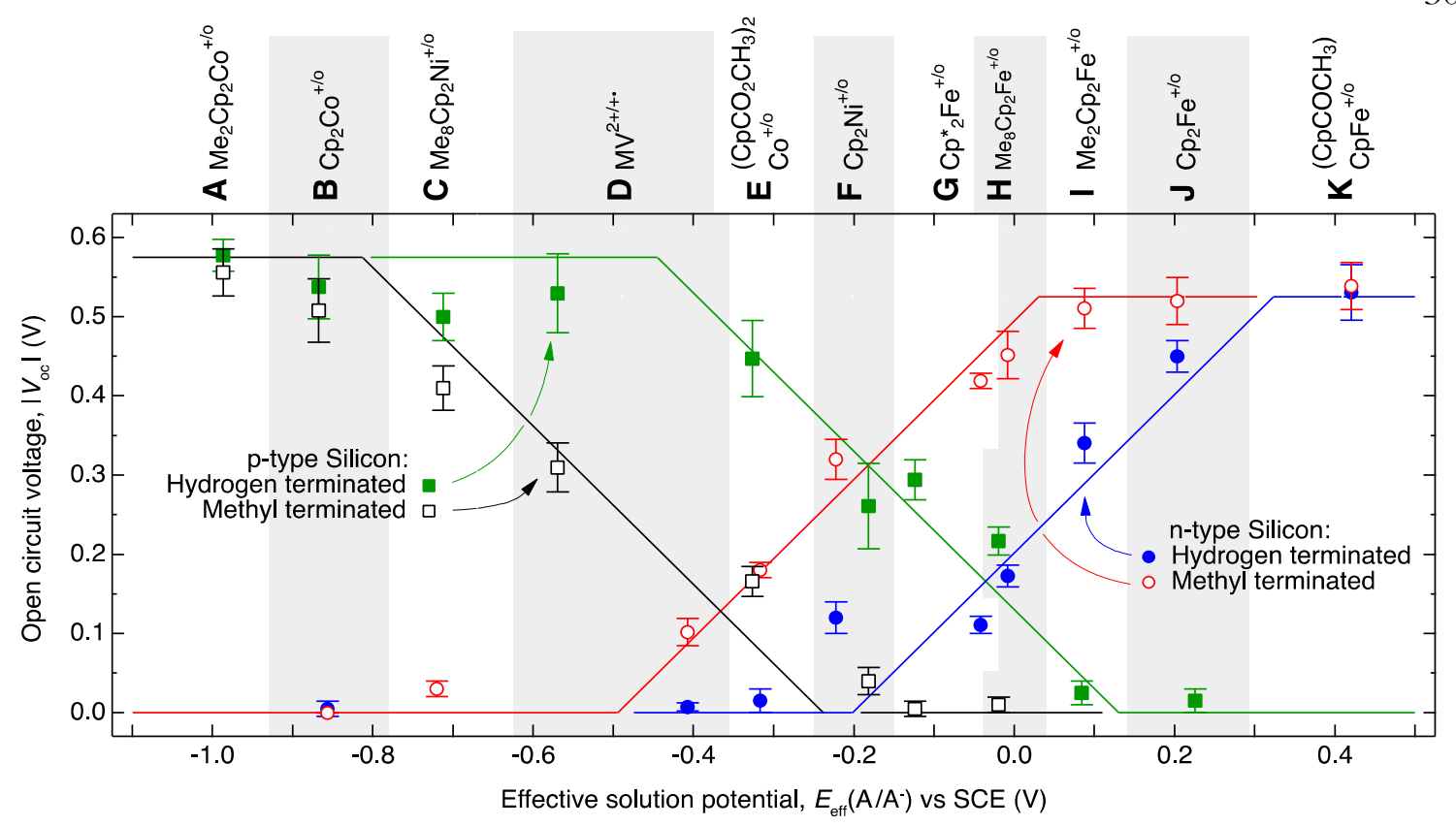

Figure 1.13 Open Circuit Voltage vs Effective Solution Potential. The open circuit voltage of $\mathrm{n}$-type and $\mathrm{p}$-type $\mathrm{Si}(111)-\mathrm{H}$ and $\mathrm{Si}(111)-\mathrm{CH}_{3}$ samples were measured using a variety of redox couples. Both n-type and p-type $\mathrm{Si}(111)-\mathrm{CH}_{3}$ samples behaved like $\mathrm{Si}(111)-\mathrm{H}$ samples that were exposed to a solution potential $0.4 \mathrm{~V}$ higher. ${ }^{48}$

Semiconductor surface states and recombination centers occasionally seem to be synonymous. Certainly in some cases they are one and the same, but there is no reason to think that they must always be both. Measurements of effective surface recombination velocities on semiconductor surfaces have clearly demonstrated that recombination can be affected by both by the concentration of recombination centers as well as band bending at the interface. ${ }^{50}$ Silicon surfaces which demonstrated long carrier lifetimes, and therefore low SRV, under band bending conditions were shown to have higher SRV measurements when redox couples in solution changed the band bending to be closer to flat band, as shown in Figure 1.14. The electric field in the space charge region prevents holes from 
getting to the surface and therefore slows the rate of recombination. Interestingly, the charged surface states that caused the initial band bending were positively charged, however the electrons present at the surface did not recombine with them to any significant degree.

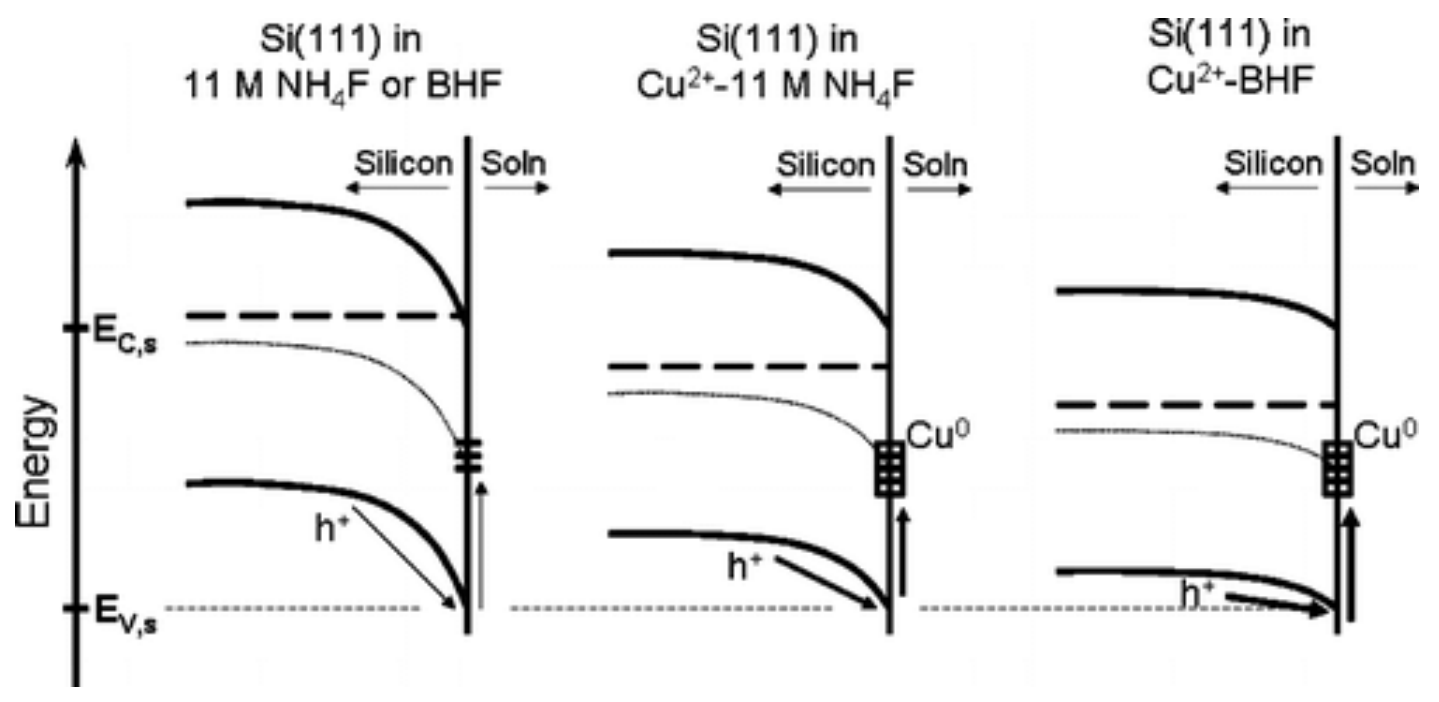

Figure 1.14 Schematic of Band Bending and Surface Recombination. At high levels of band bending, movement of holes to the surface is greatly inhibited, and without available holes, surface recombination rates are lowered. When the bands are flattened, the hole concentration at the surface is increased, as is the surface recombination velocity. This phenomenon holds true even though band bending of this nature requires positively charged surface states, indicating that these pre-existing surface states are not available for recombination with electrons at the surface. ${ }^{50}$ 


\subsection{Conclusions}

While charged surface states have been observed on semiconductors for almost as

long as semiconductors have been studied, there are still many open questions. The objective of this thesis has been to study the behavior of semiconductor surface states, albeit indirectly, to help elucidate their nature. Hopefully a better understanding of the factors controlling semiconductor interface energetics and recombination will enable development of higher efficiency semiconductor devices.

\subsection{References}

(1) Sze, S. M. Physics of Semiconductor Devices; Wiley: New York, 1981.

(2) Pierret, R. F. Advanced Semiconductor Fundamentals; 2 ed.; Pearson Education, Inc.: Upper Saddle River, New Jersey, 2003; Vol. 6.

(3) Cowley, A. M.; Sze, S. M. Surface States and Barrier Height of MetalSemiconductor Systems. J. Appl. Phys. 1965, 36 (10), 3212-3220.

(4) Eberhardt, W.; Kalkoffen, G.; Kunz, C.; Aspnes, D.; Cardona, M. Photoemission Studies of 2p Core Levels of Pure and Heavily Doped Silicon. Phys. Status Solidi B 1978, $88(1), 135-143$.

(5) Sharma, J.; Staley, R. H.; Rimstidt, J. D.; Fair, J. D.; Gora, T. F. Effect of Doping on the X-Ray Photoelectron Spectra of Semiconductors. Chem. Phys. Lett. 1971, 9 (6), 564-567.

(6) Bocarsly, A. B.; Bookbinder, D. C.; Dominey, R. N.; Lewis, N. S.; Wrighton, M. S. Photo-Reduction at Illuminated P-Type Semiconducting Silicon Photoelectrodes Evidence for Fermi Level Pinning. J. Am. Chem. Soc. 1980, 102 (11), 3683-3688. 
(7) Bard, A. J.; Bocarsly, A. B.; Fan, F. R. F.; Walton, E. G.; Wrighton, M. S. The Concept of Fermi Level Pinning at Semiconductor-Liquid Junctions Consequences for Energy-Conversion Efficiency and Selection of Useful Solution Redox Couples in Solar Devices. J. Am. Chem. Soc. 1980, 102 (11), 3671-3677.

(8) Lüth, H. Solid Surfaces, Interfaces and Thin Films; 5 ed.; Springer: New York, 2010 .

(9) Shockley, W. On the Surface States Associated with a Periodic Potential. Phys. Rev. 1939, 56 (4), 317-323.

(10) Ross, I. M. The Invention of the Transistor. Proceedings of the IEEE 1998, 86 (1), 7-28.

(11) Shockley, W. Electrons and Holes in Semiconductors; Van Nostrand: New York, 1950 .

(12) Shockley, W.; Pearson, G. L. Modulation of Conductance of Thin Films of SemiConductors by Surface Charges. Phys. Rev. 1948, 74 (2), 232-233.

(13) Meyerhof, W. E. Contact Potential Difference in Silicon Crystal Rectifiers. Phys. Rev. 1947, 71 (10), 727-735.

(14) Bardeen, J. Surface States and Rectification at a Metal Semi-Conductor Contact. Phys. Rev. 1947, 71 (10), 717-727.

(15) Gorton, W. S. The Genesis of the Transistor (Reprinted from Bell Telephone Laboratories Memorandum for Record, December 27, 1949). Proceedings of the IEEE 1998, 86 (1), 50-52.

(16) Brattain, W. H.; Shockley, W. Density of Surface States on Silicon Deduced from Contact Potential Measurements. Phys. Rev. 1947, 72 (4), 345-345.

(17) Brattain, W. H. Evidence for Surface States on Semiconductors from Change in Contact Potential on Illumination. Phys. Rev. 1947, 72 (4), 345-346. 
(18) Riordan, M.; Hoddeson, L.; Herring, C. The Invention of the Transistor. Reviews of Modern Physics 1999, 71 (2), S336-S345.

(19) Bardeen, J.; Brattain, W. H. The Transistor, a Semi-Conductor Triode. Phys. Rev. 1948, 74 (2), 230-231.

(20) Bardeen, J.; Brattain, W. H. Physical Principles Involved in Transistor Action. Phys. Rev. 1949, 75 (8), 1208-1225.

(21) Shockley, W. The Theory of P-N Junctions in Semiconductors and P-N Junction Transistors. Bell System Technical Journal 1949, 28 (3), 435-489.

(22) Brattain, W. H.; Bardeen, J. Nature of the Forward Current in Germanium Point Contacts. Phys. Rev. 1948, 74 (2), 231-232.

(23) Brattain, W. H.; Bardeen, J. Surface Properties of Germanium. Bell System Technical Journal 1953, 32 (1), 1-41.

(24) Shockley, W.; Queisser, H. J.; Hooper, W. W. Charges on Oxidized Silicon Surfaces. Phys. Rev. Lett. 1963, 11 (11), 489-490.

(25) Yu, H. B.; Webb, L. J.; Ries, R. S.; Solares, S. D.; Goddard, W. A.; Heath, J. R.; Lewis, N. S. Low-Temperature STM Images of Methyl-Terminated Si(111) Surfaces. J. Phys. Chem. B 2005, 109 (2), 671-674.

(26) Higashi, G. S.; Chabal, Y. J.; Trucks, G. W.; Raghavachari, K. Ideal Hydrogen Termination of the Si-(111) Surface. Appl. Phys. Lett. 1990, 56 (7), 656-658.

(27) Buriak, J. M. Organometallic Chemistry on Silicon and Germanium Surfaces. Chem. Rev. 2002, 102 (5), 1271-1308.

(28) Bansal, A.; Li, X. L.; Lauermann, I.; Lewis, N. S.; Yi, S. I.; Weinberg, W. H. Alkylation of Si Surfaces Using a Two-Step Halogenation Grignard Route. J. Am. Chem. Soc. 1996, 118 (30), 7225-7226. 
(29) Nemanick, E. J.; Hurley, P. T.; Brunschwig, B. S.; Lewis, N. S. Chemical and Electrical Passivation of Silicon (111) Surfaces through Functionalization with Sterically Hindered Alkyl Groups. J. Phys. Chem. B 2006, 110 (30), 14800-14808.

(30) Buriak, J. M.; Allen, M. J. Lewis Acid Mediated Functionalization of Porous Silicon with Substituted Alkenes and Alkynes. J. Am. Chem. Soc. 1998, 120 (6), 1339-1340.

(31) Webb, L. J.; Lewis, N. S. Comparison of the Electrical Properties and Chemical Stability of Crystalline Silicon(111) Surfaces Alkylated Using Grignard Reagents or Olefins with Lewis Acid Catalysts. J. Phys. Chem. B 2003, 107 (23), 5404-5412.

(32) Webb, L. J.; Rivillon, S.; Michalak, D. J.; Chabal, Y. J.; Lewis, N. S. Transmission Infrared Spectroscopy of Methyl- and Ethyl-Terminated Silicon(111) Surfaces. $J$. Phys. Chem. B 2006, 110 (14), 7349-7356.

(33) Bansal, A.; Li, X. L.; Yi, S. I.; Weinberg, W. H.; Lewis, N. S. Spectroscopic Studies of the Modification of Crystalline Si(111) Surfaces with CovalentlyAttached Alkyl Chains Using a Chlorination/Alkylation Method. J. Phys. Chem. B 2001, 105 (42), 10266-10277.

(34) Yu, H. B.; Webb, L. J.; Solares, S. D.; Cao, P. G.; Goddard, W. A.; Heath, J. R.; Lewis, N. S. Scanning Tunneling Microscopy of Ethylated Si(111) Surfaces Prepared by a Chlorination/Alkylation Process. J. Phys. Chem. B 2006, 110 (47), 23898-23903.

(35) Yu, H. B.; Webb, L. J.; Heath, J. R.; Lewis, N. S. Scanning Tunneling Spectroscopy of Methyl- and Ethyl-Terminated Si(111) Surfaces. Appl. Phys. Lett. 2006, $88(25)$.

(36) McEllistrem, M.; Haase, G.; Chen, D.; Hamers, R. J. Electrostatic Sample-Tip Interactions in the Scanning Tunneling Microscope. Phys. Rev. Lett. 1993, 70 (16), 2471-2474.

(37) Powell, C. J.; Jablonski, A. Evaluation of Calculated and Measured Electron Inelastic Mean Free Paths near Solid Surfaces. J. Phys. Chem. Ref. Data 1999, 28 (1), 19-62. 
(38) Hochella, M. F.; Carim, A. H. A Reassessment of Electron-Escape Depths in Silicon and Thermally Grown Silicon Dioxide Thin-Films. Surf. Sci. 1988, 197 (3), L260-L268.

(39) Klasson, M.; Berndtsson, A.; Hedman, J.; Nilsson, R.; Nyholm, R.; Nordling, C. Electron Escape Depth in Silicon. J. Electron Spectrosc. Relat. Phenom. 1974, 3 (6), 427-434.

(40) Jaeckel, B.; Hunger, R.; Webb, L. J.; Jaegermann, W.; Lewis, N. S. HighResolution Synchrotron Photoemission Studies of the Electronic Structure and Thermal Stability of $\mathrm{CH}_{3}$ - and $\mathrm{C}_{2} \mathrm{H}_{5}$-Functionalized $\mathrm{Si}(111)$ Surfaces. J. Phys. Chem. C 2007, 111 (49), 18204-18213.

(41) Webb, L. J.; Michalak, D. J.; Biteen, J. S.; Brunschwig, B. S.; Chan, A. S. Y.; Knapp, D. W.; Meyer, H. M.; Nemanick, E. J.; Traub, M. C.; Lewis, N. S. HighResolution Soft X-Ray Photoelectron Spectroscopic Studies and Scanning Auger Microscopy Studies of the Air Oxidation of Alkylated Silicon(111) Surfaces. $J$. Phys. Chem. B 2006, 110 (46), 23450-23459.

(42) Hunger, R.; Fritsche, R.; Jaeckel, B.; Jaegermann, W.; Webb, L. J.; Lewis, N. S. Chemical and Electronic Characterization of Methyl-Terminated Si(111) Surfaces by High-Resolution Synchrotron Photoelectron Spectroscopy. Phys. Rev. B 2005, $72(4)$.

(43) Schlaf, R.; Hinogami, R.; Fujitani, M.; Yae, S.; Nakato, Y. Fermi Level Pinning on HF Etched Silicon Surfaces Investigated by Photoelectron Spectroscopy. J. Vac. Sci. Technol., A 1999, 17 (1), 164-169.

(44) Sezen, H.; Suzer, S. Xps for Chemical- and Charge-Sensitive Analyses. Thin Solid Films 2013, 534, 1-11.

(45) Lieber, C. M.; Gronet, C. M.; Lewis, N. S. Evidence against Surface-State Limitations on Efficiency of P-Si/CH $\mathrm{CH}_{3} \mathrm{CN}$ Junctions. Nature 1984, 307 (5951), 533534.

(46) Lewis, N. S. A Quantitative Investigation of the Open-Circuit Photovoltage of the Semiconductor Liquid Interface. J. Electrochem. Soc. 1984, 131 (11), 2496-2503. 
(47) Lewerenz, H. J. Surface-States and Fermi-Level Pinning at Semiconductor Electrolyte Junctions. J. Electroanal. Chem. 1993, 356 (1-2), 121-143.

(48) Grimm, R. L.; Bierman, M. J.; O'Leary, L. E.; Strandwitz, N. C.; Brunschwig, B. S.; Lewis, N. S. Comparison of the Photoelectrochemical Behavior of $\mathrm{H}-$ Terminated and Methyl-Terminated Si(111) Surfaces in Contact with a Series of One-Electron, Outer-Sphere Redox Couples in $\mathrm{CH}_{3} \mathrm{CN}$. Journal of Physical Chemistry C 2012, 116 (44), 23569-23576.

(49) Walter, M. G.; Warren, E. L.; McKone, J. R.; Boettcher, S. W.; Mi, Q. X.; Santori, E. A.; Lewis, N. S. Solar Water Splitting Cells. Chem. Rev. 2010, 110 (11), 64466473.

(50) Michalak, D. J.; Gstrein, F.; Lewis, N. S. The Role of Band Bending in Affecting the Surface Recombination Velocities for $\mathrm{Si}(111)$ in Contact with Aqueous Acidic Electrolytes. Journal of Physical Chemistry C 2008, 112 (15), 5911-5921. 


\title{
Chapter 2
}

\section{Measurement of the Band Bending and Surface Dipole at Chemically Functionalized $\mathrm{Si}(111)$ /Vacuum Interfaces}

\author{
Reproduced from Gleason-Rohrer, D. C.; Brunschwig, B. S.; Lewis, N. S. \\ J. Phys. Chem. C 2013, 117(35), 18031-18042.
}

\subsection{Abstract}

The core-level energy shifts observed using X-ray photoelectron spectroscopy (XPS) have been used to determine the band bending at $\operatorname{Si}(111)$ surfaces terminated with $\mathrm{Si}-\mathrm{Br}, \mathrm{Si}-\mathrm{H}$, and $\mathrm{Si}-\mathrm{CH}_{3}$ groups, respectively. The surface termination influenced the band bending, with the Si $2 p_{3 / 2}$ binding energy affected more by the surface chemistry than by the dopant type. The highest binding energies were measured on $\mathrm{Si}(111)-\mathrm{Br}$ (whose Fermi level was positioned near the conduction band at the surface), followed by $\mathrm{Si}(111)-\mathrm{H}$, followed by $\mathrm{Si}(111)-\mathrm{CH}_{3}$ (whose Fermi level was positioned near mid-gap at the surface). $\mathrm{Si}(111)-\mathrm{CH}_{3}$ surfaces exposed to $\mathrm{Br}_{2}(\mathrm{~g})$ yielded the lowest binding energies, with the Fermi level positioned between mid-gap and the valence band. The Fermi level position of $\mathrm{Br}_{2}(\mathrm{~g})$-exposed $\mathrm{Si}(111)-\mathrm{CH}_{3}$ was consistent with the presence of negatively charged bromine-containing ions on such surfaces. The binding energies of all of the species detected on the surface $(\mathrm{C}, \mathrm{O}, \mathrm{Br})$ shifted with the band bending, illustrating the importance of isolating the effects of band bending when measuring chemical shifts on semiconductor surfaces. The influence of band bending was confirmed by surface photovoltage (SPV) measurements, which showed that the core levels shifted toward their flat-band values upon illumination. Where applicable, the contribution from the X-ray source to the SPV was 
isolated and quantified. Work functions were measured by ultraviolet photoelectron spectroscopy (UPS), allowing for calculation of the sign and magnitude of the surface dipole in such systems. The values of the surface dipoles were in good agreement with previous measurements as well as with electronegativity considerations. The binding energies of the adventitious carbon signals were affected by band bending as well as by the surface dipole. A model of band bending in which charged surface states are located exterior to the surface dipole is consistent with the XPS and UPS behavior of the chemically functionalized $\mathrm{Si}(111)$ surfaces investigated herein.

\subsection{Introduction}

Band bending ${ }^{1}$ induced by charged surface states has been demonstrated to influence, and sometimes dominate, the device characteristics of semiconductor surfaces, including the surface barrier height, ${ }^{2}$ surface conductivity, surface recombination velocity, and surface photochemistry. ${ }^{3}$ As devices become smaller, these surface effects play an even larger role. The effects of band bending have been well documented for silicon/metal contacts, ${ }^{2,4}$ silicon/solution contacts, ${ }^{5-8}$ and silicon/vacuum contacts. ${ }^{9-10}$ In many cases, the band bending is different than what is expected from a simple comparison of the work functions of the contacting materials. During the charge equilibration process, surface states with donor or acceptor levels in the band gap become emptied or filled, respectively, until the highest occupied donor/lowest unoccupied acceptor states are positioned at the Fermi level. The charge equilibration process results in trapped majority carriers in the surface states and a depletion layer inside the 
semiconductor. These trapped charges generate an electric field within the semiconductor. If the concentration of donors or acceptors is sufficient to dominate the position of the Fermi level regardless of the contacting material or dopant type and dopant density of the semiconductor, then the contact can be described as Fermi level pinned. In vacuum, any band bending observed can only be ascribed to charged surface states, measured as the surface state charge density $Q_{s s}$, and so any deviation from the flat-band condition would be due to Fermi level pinning.

Identification of the nature of the states that contribute to $Q_{s s}$ is typically complicated by the low concentration of such surface state species. For example, for moderately doped silicon with a relatively high concentration of net surface states, sufficient to produce band bending of $0.7-0.8 \mathrm{eV}$, that spans the entire band gap, the total charge would correspond to only $\sim 0.02 \%$ of all surface atoms. Among other possibilities, these surface states have been ascribed to surface unit cells diverging from bulk cell properties, dangling bonds, metal-induced gap states, surface reconstruction, step edges or dislocations, impurity atoms, and/or adsorbed surface ions. ${ }^{11}$ All of these effects could contribute together to surface states. However, the surface states at the highest occupied donor/lowest unoccupied acceptor energy level will determine the ultimate energy of the Fermi level with respect to the valence-band maximum at the surface $\left(\mathbf{E}_{\mathrm{vbm}}^{\mathbf{s}}\right)$. Historically, impurity atoms and adsorbed ions have been discounted due to their assumed independence from the band bending, ${ }^{12}$ with adsorbed ions generally treated as fixed charges without energy levels in the band gap. 
X-ray photoelectron spectroscopy (XPS) has been used to determine the band bending of semiconductors in ultrahigh vacuum (UHV). The binding energy, BE, determined by XPS is a measure of the difference in energy between the elemental core level and the Fermi level. While at equilibrium the Fermi level is flat (i.e., at constant energy), the core levels as well as the valence and conduction levels will bend up or down in unison due to the presence of the electric field in the space-charge region of the semiconductor. The escape depth of photogenerated electrons in crystalline silicon ranges ${ }^{13}$ from $1.6 \mathrm{~nm}^{14}$ to over $3.9 \mathrm{~nm}^{15}$ for a $1387 \mathrm{eV}$ electron. For XP spectra, when the space-charge region is very large compared to the escape depth of the photogenerated electron, as is the case for moderately doped $\mathrm{Si}$, then all of the detected electrons will have a relatively narrow energy distribution. Further, their binding energy will be shifted from that calculated for the bulk (i.e., flat band) by the band-bending energy $\mathbf{E}_{\mathrm{bb}}$ (in other literature sources $e V_{\mathrm{bb}}$ is used instead). Alternatively, if the escape depth of the photogenerated electrons is approximately the same as the depletion width, then the detected electrons will have a range of energies, due to broadening by the spatially dependent electric potential in the space-charge region of the semiconductor. ${ }^{16}$

The presence of a surface photovoltage $(\mathrm{SPV})^{12}$ can help verify that an observed binding energy shift is caused by band bending. Although most work on SPV has used Kelvin probe measurements, ${ }^{12,17}$ XPS has also been used. ${ }^{18-19}$ Upon illumination, charge carriers are generated in the bulk and in the depletion region. Minority carriers generated in the depletion region will move to the surface via drift due to the electric field, while majority carriers in the depletion region will move into the bulk. Minority carriers 
generated in the bulk close to the depletion region will move toward the depletion region via diffusion. A steady state will be established between minority carriers coming to the surface, recombination, and surface state charging/discharging processes. This steady state will result in a lower effective surface-state charge density and therefore will reduce the band bending. The difference between the binding energy observed in the dark and under illumination will give the SPV. This difference should be smaller than or equal to the initial band bending, $\mathbf{E}_{\mathrm{bb}}$. Illumination cannot reduce the band bending to the flat band condition unless the rate of surface recombination is very much lower than the rate of minority-carrier diffusion and electron-hole generation by the light. The SPV effect can also occur in the absence of deliberate illumination of the sample, due to carrier excitation from the X-ray source, and such effects can possibly mask the intended SPV experiment. ${ }^{16}$

The surface-based electronic properties (band bending, work function, and dipole) have been studied and characterized on numerous Si surfaces. Three broad classes of modification include deposition of inorganic thin films, ${ }^{20-23}$ of redox couples, 17, 24-28 and of molecules. ${ }^{29-32}$ Comparisons between different surface modifications, even in the same class, are challenging due to variations in Si bond formation vs adsorption, in the fraction of atop sites affected by the modification, and in the degree of Si oxidation during modification, as well as due to the presence of unreacted sites. ${ }^{33}$

It is well-documented that p-type $\mathrm{Si}(111)-\mathrm{H}$ surfaces exhibit band bending. ${ }^{16,24-}$ 26, 28, 32, 34-35 For both n-type and p-type Si(111)-H surfaces in vacuum, the Fermi level is 
positioned at $0.7-0.9 \mathrm{eV}$ above the valence band at the surface $\left(\mathbf{E}_{\mathrm{vbm}}^{\mathbf{S}}=0.7-0.9 \mathrm{eV}\right)$. Most molecular attachment techniques, particularly those using sterically hindered groups, leave residual $\mathrm{Si}-\mathrm{H}$ bonds, and these residual bonds can dominate the bandbending properties after further modification. The majority of work on molecularly modified Si surfaces, with and without redox couples, indicates minimal $(<0.1 \mathrm{eV})$ change in band bending relative to the behavior of the Si-H surface. ${ }^{24,26,28-29,32,36-37}$

Complicating analysis, the band banding on $\mathrm{Si}$ surfaces is not necessarily constant. Si $2 \mathrm{p}_{3 / 2}$ binding energies on single $\mathrm{p}-\mathrm{Si}(111)-\mathrm{H}$ samples have been shown to change with annealing. ${ }^{35}$ The $\mathrm{Si} 2 \mathrm{p}_{3 / 2}$ binding energies on single $\mathrm{n}$-Si(100) samples with a thin $\mathrm{SiO}_{2}$ layer were observed to shift to higher binding energy values, indicating increased $\mathbf{E}_{\mathrm{vbm}}^{\mathbf{s}}$, due to X-ray irradiation from the XPS source. This behavior was attributed to carrier trapping at the interface ${ }^{20}$ and correlated with the trace amounts of residual $\mathrm{Si}-\mathrm{H}$ bonds on some surfaces. ${ }^{22}$ Kelvin probe measurements have demonstrated that the band bending of a single sample of alkylated p-Si(100) can reversibly change over time, due to charge trapping caused by 848 - and $670-\mathrm{nm}$ illumination. ${ }^{38}$

Given these considerations, proper band-bending analysis requires the following: measurement on multiple samples to obtain an appropriate statistical distribution, dark conditions with minimal SPV generated from the X-ray source, minimal X-ray exposure prior to core level analysis, consistent $\mathbf{E}_{\mathrm{vbm}}^{\mathrm{s}}$ positions on moderately doped samples of both dopant types, and proper instrument Fermi level calibration at energies near the core levels of interest (preferably using the positions of the $\mathrm{Ag} 3 \mathrm{~d}$ and $\mathrm{Au} 4 \mathrm{f}$ peaks as 
standards). Furthermore, a surface modification known to completely eliminate $\mathrm{Si}-\mathrm{H}$ bonds can be useful for isolating the known positioning/pinning of the $\mathrm{Si}-\mathrm{H}$ surface from other characteristics affecting band bending.

Grignard chemistry has been well developed to introduce covalent Si-C functionality to the atop sites of the $\operatorname{Si}(111)$ surface. $^{33}{ }^{39-41}$ Sterically unhindered groups such as methyl have been demonstrated to provide nearly complete termination of atop sites on an unreconstructed $\operatorname{Si}(111)$ surface, ${ }^{42}$ as well as to prevent oxidation in air and in solution and to maintain low surface recombination velocities. ${ }^{41}$ Gas-phase bromination ${ }^{43}$ allows for the formation of polar $\mathrm{Si}^{\delta+}-\mathrm{Br}^{\delta-}$ on $\mathrm{Si}(111)$ surfaces. Hence, these techniques provide tools for observing how changes in the surface chemical environment affect the surface band bending.

In this work, gas-phase bromination and small-molecule Grignard chemistry have been used to prepare $\operatorname{Si}(111)$ surfaces that have $100 \%$ surface coverage as well as relatively large differences between the values of the surface dipoles. The resulting band-bending behavior has been considered in the context of the possible location of the charged surface states relative to the surface dipole. The shifts in binding energy of adventitious carbon have been analyzed, and the dipole contribution to the energy shift has been isolated. These surfaces therefore provide a model system for consideration of the effects of redox couples adsorbed onto semiconductor surfaces. 


\subsection{Experimental section}

\subsubsection{Materials}

Methanol (VWR), acetone (VWR), hydrogen peroxide (30\%, VWR), sulfuric acid (VWR), dichloromethane (low evaporation residue, Aldrich), and tetrahydrofuran (anhydrous, Aldrich) were used as received. Water with a resistivity of $18 \mathrm{M} \cdot \Omega \mathrm{cm}$ was obtained from a Barnsted Nanopure system. Bromine (Aldrich) was collected by distillation from a 1:1 mixture of bromine:sulfuric acid into a Schlenk flask, followed by a freeze-pump-thaw procedure, and was stored under $\operatorname{Ar}(\mathrm{g})$ prior to use. $\mathrm{CH}_{3} \mathrm{MgCl}, 3.0$ $\mathrm{M}$ in THF (Aldrich), was used as received, but only was used for a maximum of two weeks after the SureSeal top had been punctured. HF(aq) (48\%, Transene Inc.) was used as received, and $\mathrm{NH}_{4} \mathrm{~F}(\mathrm{aq})(40 \%$, Transene Inc.) was degassed with $\mathrm{Ar}(\mathrm{g})$ for 30 min prior to use.

Single-side polished, float zone, (111)-oriented Si wafers with a miscut angle of $\pm 0.5^{\circ}$ had the following specifications: p-Si(111), $11-18 \Omega \cdot \mathrm{cm}$, boron-doped, $\mathbf{E}_{\mathrm{vbm}}^{\mathrm{B}}=$ $0.27 \mathrm{eV} ; \mathrm{n}-\mathrm{Si}(111), 0.8-1.5 \Omega \cdot \mathrm{cm}$, phosphorous-doped, $\mathbf{E}_{\mathrm{vbm}}^{\mathrm{B}}=0.88 \mathrm{eV} ; \mathrm{p}^{+}-\mathrm{Si}(111)$ 0.001-0.002 $\Omega \cdot \mathrm{cm}$, boron-doped, $\mathbf{E}_{\mathrm{vbm}}^{\mathrm{B}}=0.09 \mathrm{eV} ; \mathrm{n}^{+}-\mathrm{Si}(111), \quad 0.005-0.02 \Omega \cdot \mathrm{cm}$, phosphorous-doped, $\mathbf{E}_{\mathrm{vbm}}^{\mathrm{B}}=1.02 \mathrm{eV} ; \mathrm{n}^{++}-\mathrm{Si}(111), 0.002-0.004 \Omega \cdot \mathrm{cm}$, phosphorous-doped, $\mathbf{E}_{\mathrm{vbm}}^{\mathrm{B}}=1.08 \mathrm{eV}$. Samples were cut into $\sim 8$-mm x 8-mm pieces. 


\subsubsection{Surface Chemistry}

Samples were cleaned by sequential rinses in streams of $\mathrm{H}_{2} \mathrm{O}, \mathrm{CH}_{3} \mathrm{OH}$, acetone, $\mathrm{CH}_{3} \mathrm{OH}$, and $\mathrm{H}_{2} \mathrm{O}$. Samples were then placed in a solution of $1: 1 \mathrm{H}_{2} \mathrm{O}_{2}: \mathrm{H}_{2} \mathrm{SO}_{4}$ for a minimum of 30 min, rinsed with $\mathrm{H}_{2} \mathrm{O}$, submerged for 1 min in $\mathrm{HF}$, rinsed with $\mathrm{H}_{2} \mathrm{O}$, and submerged for $5 \mathrm{~min}$ in degassed $\mathrm{NH}_{4} \mathrm{~F}$. Etched samples were sonicated for $20 \mathrm{~s}$ in $\mathrm{H}_{2} \mathrm{O}$ and then for $20 \mathrm{~s}$ in $\mathrm{CH}_{2} \mathrm{Cl}_{2}$. Samples were then dried under $\mathrm{N}_{2}(\mathrm{~g})$ and immediately placed under vacuum using a Schlenk line. The elapsed time between removal of the sample from the etching solution and placement of the sample under vacuum was typically $2 \mathrm{~min}$.

Gas-phase bromination was performed on a Schlenk line. After a minimum of three $\operatorname{Ar}(\mathrm{g})$ backfills, the sample flask was returned to vacuum and opened to a flask that contained liquid $\mathrm{Br}_{2}$. Red-maroon bromine gas visibly filled the sample flask, and the bromine flask was resealed after $1 \mathrm{~s}$. For samples that were to be subsequently methylated, the sample and bromine were exposed to UV light for $1 \mathrm{~min}$, with a total reaction time of $5 \mathrm{~min}$. For samples that were brominated immediately before analysis by XPS ( $\mathrm{Si}(111)-\mathrm{Br}$ and $\left.\mathrm{Si}(111)-\mathrm{CH}_{3}[\mathrm{Br}]\right)$, the sample and bromine were kept in the dark and reacted for a total of $1 \mathrm{~min}$, after which the sample was returned to vacuum.

To methylate the Si surfaces, while under a positive pressure of argon, the Brterminated $\mathrm{Si}(111)$ surface was submerged in 3.0-M $\mathrm{CH}_{3} \mathrm{MgCl}$ in $\mathrm{THF}$ and heated for 90 min at $90{ }^{\circ} \mathrm{C}$. After cooling, the samples were exposed to air, rinsed, and sonicated sequentially in THF, $\mathrm{H}_{2} \mathrm{O}$, and $\mathrm{CH}_{2} \mathrm{Cl}_{2}$ and then dried under $\mathrm{N}_{2}(\mathrm{~g})$. All samples were 
transferred through air to the load lock of the UHV chamber. Figure 2.1 provides a graphical depiction of the process flow.

HF: 1 min

Degassed $\mathrm{NH}_{4} \mathrm{~F}: 5 \mathrm{~min}$

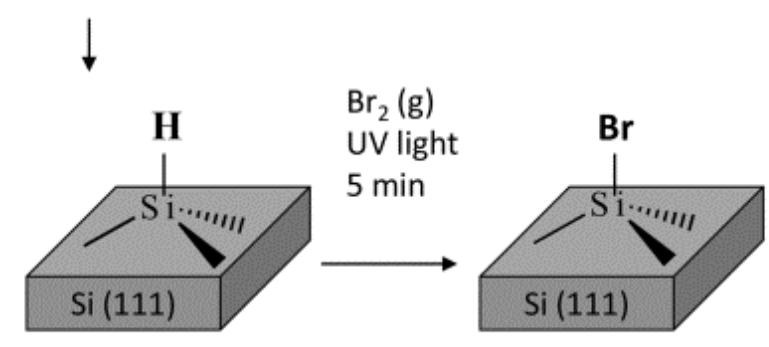

Cleaning:

Sonicated in $\mathrm{H}_{2} \mathrm{O}$ then DCM

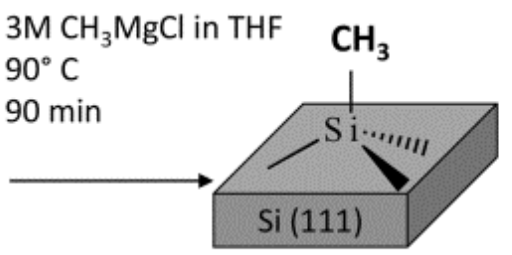

Si(111)-H

Cleaning:

Sonicated in THF, $\mathrm{H}_{2} \mathrm{O}$, then DCM

$\mathrm{Si}(111)-\mathrm{CH}_{3}$

$\mathrm{Br}_{2}(\mathrm{~g})$
dark
1 min
No rinse

$\mathrm{Br}_{2}(\mathrm{~g})$

dark

$1 \mathrm{~min}$

$\mathrm{Si}(111)-\mathrm{Br}$

No rinse

$\mathrm{Si}(111)-\mathrm{CH}_{3}[\mathrm{Br}]$

Figure 2.1 Schematic Representation of Si(111) Surface Functionalization.

\subsubsection{Infrared Spectroscopy}

All spectra were collected on a Thermo Scientific Nicolet 6700 Optical Spectrometer with a DTGS-TEC detector and a purified-air purge. Double sided polished, n-type phosphorous-doped wafers with a resistivity of $63-77 \Omega \cdot \mathrm{cm}$ and a thickness of $450-430 \mu \mathrm{m}$ were cut to $\sim 1.6 \mathrm{~cm} \times 3 \mathrm{~cm}$ and prepared as described above. Samples of this type were used only for IR analysis, but XPS analysis was also performed on them, and their surface properties were found to be consistent with the other samples 
presented. After the surface chemistry was completed, the samples were positioned at an angle of $76.4^{\circ}$ from normal to the IR beam. Background transmission spectra of the Si(111)-H surface were collected for each wafer prior to any further surface modification and were used as a background for the other samples. Spectra were collected with $4 \mathrm{~cm}^{-1}$ resolution after 40 min of purging. ${ }^{44}$

\subsubsection{Photoelectron Spectroscopy}

All spectra were collected on a Kratos Axis Ultra system (base pressure $10^{-9}$ torr), with a monochromatic $\mathrm{Al} \mathrm{K} \alpha \mathrm{X}$-ray source. The X-ray source power either was set at 10 $\mathrm{W}$ (to minimize SPV effects) for $\mathrm{Si} 2 \mathrm{p}$ regions marked as such or for all other regions was set at $150 \mathrm{~W}$ (to maximize signal-to-noise). A pass energy of $5 \mathrm{eV}$ and a step energy of $0.025 \mathrm{eV} \mathrm{step}^{-1}$ were used for the $10-\mathrm{W}$ power experiments, a $40-\mathrm{eV}$ pass energy and a step energy of $1.0 \mathrm{eV} \mathrm{step}^{-1}$ were used for survey scans, and all other spectra were collected using a $10-\mathrm{eV}$ pass energy and a step energy of $0.025 \mathrm{eV}^{-1}$ step $^{-1}$. The photoelectrons were collected at the surface normal by use of a hemispherical analyzer, with no charge neutralization. The photoemission energy scale was calibrated using freshly sputtered films of either $\mathrm{Au}$ or $\mathrm{Ag}$, and the energy scale was confirmed to be within $0.05 \mathrm{eV}$ of standards or was recalibrated $(\mathrm{Ag} 3 \mathrm{~d}=368.26 \pm 0.02 \mathrm{eV}, \mathrm{Au} 4 \mathrm{f}=$ $84.27 \pm 0.02 \mathrm{eV})$ at 2 -week intervals. Samples were measured in the dark unless otherwise noted. External illumination was provided by an unfocused $150-\mathrm{W}$ halogen lamp that was positioned $20 \mathrm{~cm}$ from the sample (outside the UHV chamber) at an incident angle of $30^{\circ}$. Secondary electron cutoff measurements were acquired in the 
same UHV chamber by use of He I $(h v=21.2 \mathrm{eV})$ radiation. No voltage was applied to the sample during either the XPS or the UPS measurements.

All peaks were fit (with CasaXPS software ${ }^{45}$ ) to symmetric Voigt line shapes that were $70 \%$ Gaussian and $30 \%$ Lorentzian product functions. The Si 2 p regions were fit to two peaks, and the $\mathrm{C}$ 1s regions were fit to two peaks for nonmethylated samples and to three peaks for methylated samples. The peak positions were much less sensitive to the fitting parameters than were the relative peak areas; hence, any error associated with the fit was considerably less than the variation in binding energy observed between different samples of nominally the same surface composition.

\subsubsection{Band Bending, $\mathbf{E}_{\mathrm{vbm}}$, Work Function, and Dipole Determination ${ }^{32}$}

In XPS, the momentum of the emitted photoelectrons is measured at a detector. The difference between the X-ray excitation energy $(h v)$ and the sum of the kinetic energy of the photoelectron at the surface plus the sample work function gives the binding energy $(\mathrm{BE})$, which is the difference in energy from the core level to the Fermi level. The core level, valence band, and conduction band all bend in unison, so changes to $\mathbf{E}_{\mathrm{vbm}}^{\mathbf{s}}$ (energy difference from the valence band to the Fermi level at the surface) are mirrored by changes in BEs. While the work function of the sample $\left(W_{\mathrm{F}, \mathrm{s}}\right)$ affects the kinetic energy of the electrons at the sample surface $\left(\mathrm{KE}_{\mathrm{s}}\right)$, the sum of the kinetic energy at the detector $\left(\mathrm{KE}_{\mathrm{d}}\right)$ and the detector work function $\left(W_{\mathrm{F}, \mathrm{d}}\right)$ equals the sum of $\mathrm{KE}_{\mathrm{s}}$ and $W_{\mathrm{F}, \mathrm{s}}{ }^{46}$ Thus the binding energy is given by Eq 2.1,

$$
\mathrm{BE}=h v-\left(\mathrm{KE}_{\mathrm{d}}+W_{\mathrm{F}, \mathrm{d}}\right)
$$


$W_{\mathrm{F}, \mathrm{s}}$, and consequently a surface dipole $(\delta)$, will not directly affect the binding energy of photoelectrons generated interior to the dipole (Figure 2.2). Surface functionalization treatments will result in different surface dipoles, $W_{\mathrm{F}, \mathrm{s}}$, and band bending. While changes in $W_{\mathrm{F}, \mathrm{s}}$ do not affect the observed $\mathrm{BE}$, changes in the band bending will affect the observed BE.

Alternatively, the binding energy of photoelectrons generated exterior to a surface dipole will be shifted by the dipole (Figure 2.2b). The core levels of adventitious material will be shifted by the initial position of the Fermi level within the band gap, as well as by the band bending and any surface dipole. Hence, the adventitious carbon signal cannot be used as a binding energy reference for analysis of the energy levels of the semiconductor.

The binding energy, using $\mathrm{Au}$ as a Fermi level reference, of the $\mathrm{Si} 2 \mathrm{p}_{3 / 2}$ peak of bulk crystalline $\mathrm{Si}$ with the Fermi level pinned to the valence band, referred to as $\mathrm{BE}^{\mathrm{V}}(\mathrm{Si}$ $2 \mathrm{p}_{3 / 2}$ ), was determined previously to be $98.74 \mathrm{eV},{ }^{21}$ allowing the measured binding energies to be placed precisely within the band gap. Samples with the Fermi level pinned to the conduction band were also previously measured and were found to be in good agreement with a 1.12-eV band gap. Properly grounded/calibrated Si $2 \mathrm{p}_{3 / 2}$ measurements should therefore fall within the range between 98.74 and $99.86 \mathrm{eV}$. The energy difference from the valence band to the Fermi level at the surface can be determined for moderately doped Si as

$$
\mathbf{E}_{\mathrm{vbm}}^{\mathbf{s}}=\mathrm{BE}\left(\mathrm{Si} 2 \mathrm{p}_{3 / 2}\right)-\mathrm{BE}^{\mathrm{V}}\left(\mathrm{Si} 2 \mathrm{p}_{3 / 2}\right)
$$


Figure 2.2 Energy Level Diagram of Silicon in an XPS Demonstrating Dipole Shift
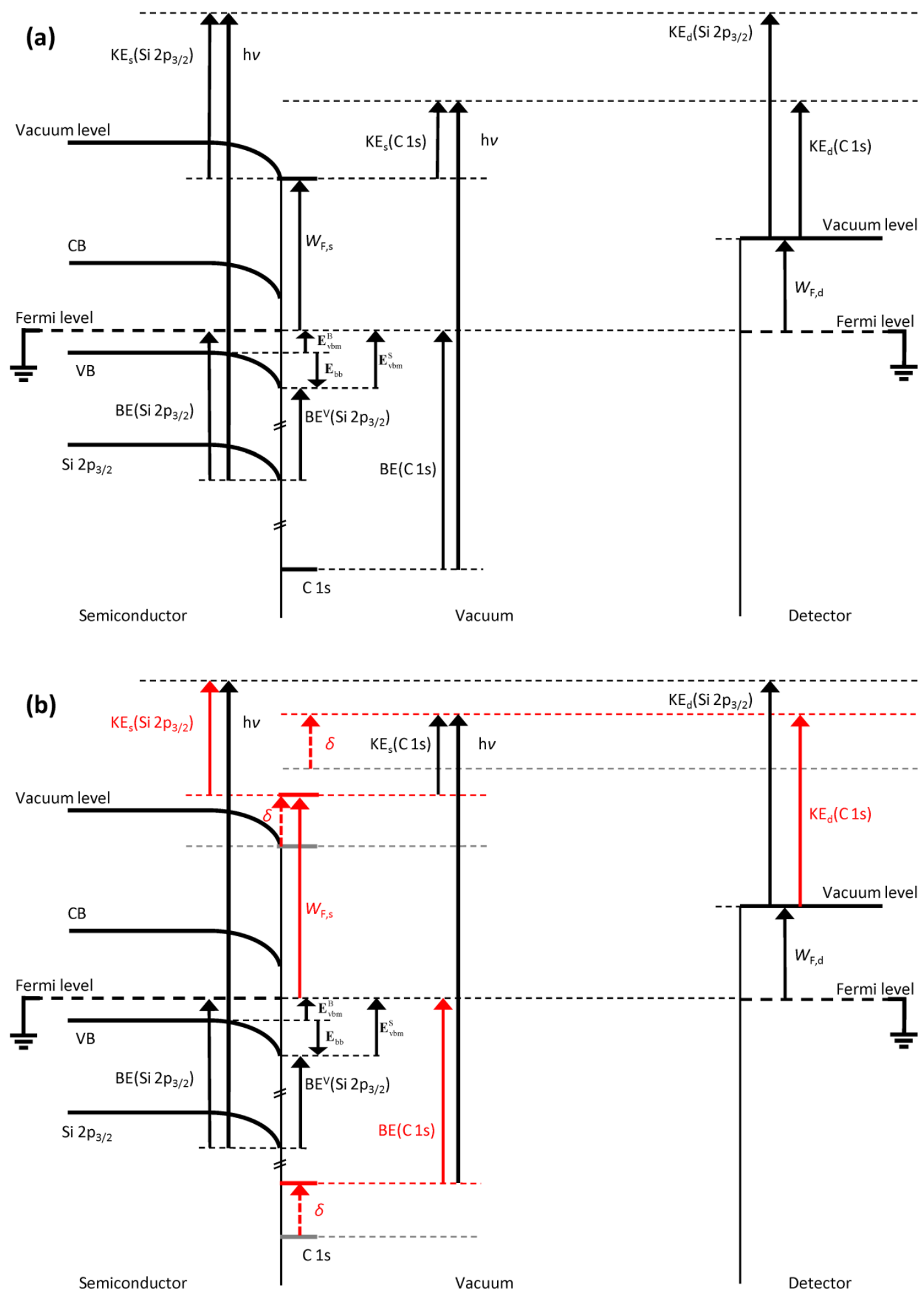
Figure 2.2 Energy Level Diagram of Silicon in an XPS Demonstrating Dipole Shift. Energy level diagram of p-type silicon in an XPS shown (a) without and (b) with a surface dipole, $\delta$. The red or dark gray marking in (b) indicate energy levels or values that are affected by $\delta$, while the previous locations for the energy levels are depicted in light gray. $W_{\mathrm{F}, \mathrm{s}}$ and $W_{\mathrm{F}, \mathrm{d}}$ are the work functions of the Si sample and of the detector, respectively, $h v$ is the X-ray energy. $\mathrm{BE}\left(\mathrm{Si} 2 \mathrm{p}_{3 / 2}\right)$ and $\mathrm{BE}(\mathrm{C} 1 \mathrm{~s})$ are binding energies of the $\mathrm{Si} 2 \mathrm{p}_{3 / 2}$ and $\mathrm{C} 1 \mathrm{~s}$ core levels, respectively, where the adventitious $\mathrm{C}$ is located exterior to $\delta$ and the $\mathrm{Si}$ is located interior to $\delta$. $\mathrm{BE}^{\mathrm{V}}\left(\mathrm{Si} 2 \mathrm{p}_{3 / 2}\right)$ is the energy difference between the $\mathrm{Si} 2 \mathrm{p}_{3 / 2}$ core level and the valence-band maximum. $\quad \mathbf{E}_{\mathrm{vbm}}^{\mathrm{B}}$ and $\mathbf{E}_{\mathrm{vbm}}^{\mathbf{s}}$ are the energies of the Fermi level with respect to the valence-band maximum in the bulk and at the surface, respectively. $\mathbf{E}_{\mathrm{bb}}$ is the band bending energy, and the arrow is pointing down because the value of $\mathbf{E}_{\mathrm{bb}}$ is negative as drawn. $\mathrm{KE}_{\mathrm{s}}$ is the kinetic energy of the photoelectrons emitted from the indicated core levels at the surface of the sample (exterior to the dipole). $\mathrm{KE}_{\mathrm{d}}$ is the kinetic energy of the photoelectrons as measured at the detector. The valence (VB) and conduction (CB) bands of silicon are shown. Note that the dipole, $\delta$, will not directly affect the $\mathrm{Si} 2 \mathrm{p}_{3 / 2}$ binding energy measurement, but could have an indirect effect if the dipole/surface treatment changes the surface charge density, and consequently the band bending.

The degree of band bending can be determined by taking the difference in energy of the Fermi level relative to the valence band in the bulk $\left(\mathbf{E}_{\mathrm{vbm}}^{\mathrm{B}}\right)$, as determined from dopant density calculations, minus $\mathbf{E}_{\mathrm{vbm}}^{\mathbf{s}}$. Note that positive $\mathbf{E}_{\mathrm{bb}}$ indicates that the band levels increase in energy relative to the Fermi level as one moves from the bulk to the surface,

$$
\mathbf{E}_{\mathrm{bb}}=\mathbf{E}_{\mathrm{vbm}}^{\mathrm{B}}-\mathbf{E}_{\mathrm{vbm}}^{\mathrm{s}}=\mathbf{E}_{\mathrm{vbm}}^{\mathrm{B}}+\mathrm{BE}^{\mathrm{V}}\left(\mathrm{Si} 2 \mathrm{p}_{3 / 2}\right)-\mathrm{BE}\left(\mathrm{Si} 2 \mathrm{p}_{3 / 2}\right)
$$

The work function at the surface $W_{\mathrm{F}, \mathrm{s}}$, which is dependent on both the band bending and the surface dipole, as shown in Figure 2.2b, was determined from the UPS spectra. The secondary electron cutoff was measured as the energy position of the half- 
height of the intensity determined from a straight line fit of the cutoff data, with $W_{\mathrm{F}, \mathrm{s}}$ determined by

$$
W_{\mathrm{F}, \mathrm{s}}=h v-\mathbf{E}_{\mathrm{cutoff}}
$$

where $h v$ is the He I emission at $21.2 \mathrm{eV}$. The surface dipole $(\delta)$ can be calculated as the difference between the surface electron affinity $\left(\chi^{S}\right)$ and the bulk electron affinity $\left(\chi^{\mathrm{B}}\right)$, which has a nominal value of $4.05 \mathrm{eV},{ }^{1,30}$

$$
\begin{aligned}
& \chi^{\mathrm{S}}=W_{\mathrm{F}, \mathrm{s}}-\mathbf{E}_{\mathrm{cbm}}^{\mathrm{S}}=W_{\mathrm{F}, \mathrm{s}}-\left(\mathbf{E}_{\mathrm{g}}-\mathbf{E}_{\mathrm{vbm}}^{\mathrm{S}}\right), \\
& \delta=\chi^{\mathrm{S}}-\chi^{\mathrm{B}}=W_{\mathrm{F}, \mathrm{s}}-\mathbf{E}_{\mathrm{g}}+\mathbf{E}_{\mathrm{vbm}}^{\mathrm{S}}-\chi^{\mathrm{B}},
\end{aligned}
$$

where $\mathbf{E}_{\mathrm{g}}$ is the band gap of silicon, i.e., $\mathbf{E}_{\mathrm{g}}=1.12 \mathrm{eV}$. Both $\mathbf{E}_{\mathrm{vbm}}^{\mathrm{s}}$ and $W_{\mathrm{F}, \mathrm{s}}$ are individually dependent on $\mathbf{E}_{\mathrm{bb}}$; however, the sum of $\mathbf{E}_{\mathrm{vbm}}^{\mathrm{s}}$ and $W_{\mathrm{F}, \mathrm{s}}$ is independent of $\mathbf{E}_{\mathrm{bb}}$, giving a value for the dipole that is independent of the band bending. Hence, measurements in the light and in the dark should give the same value for the dipole; however, in practice, differences due to X-ray and/or UV photon-generated SPV may cause a discrepancy between the values determined by the two different approaches. Table 2.1 summarizes the calculations for the relevant calculated quantities.

\section{Table 2.1 Quantities Calculated}

$$
\begin{gathered}
\mathbf{E}_{\mathrm{vbm}}^{\mathrm{S}}=B E\left(\operatorname{Si} 2 \mathrm{p}_{3 / 2}\right)-B E^{\mathrm{V}}\left(\mathrm{Si} 2 \mathrm{p}_{3 / 2}\right)^{1} \\
\mathbf{E}_{\mathrm{bb}}=\left(\mathbf{E}_{\mathrm{vbm}}^{\mathrm{B}}+B E^{\mathrm{V}}\left(\operatorname{Si} 2 p_{3 / 2}\right)\right)-B E\left(\operatorname{Si} 2 p_{3 / 2}\right) \\
W_{\mathrm{F}, \mathrm{s}}=\mathrm{h} v-\mathbf{E}_{\text {cutoff }} 2 \\
\delta=\chi^{\mathrm{S}}-\chi^{\mathrm{B}}=\left(W_{\mathrm{F}, \mathrm{s}}-\mathbf{E}_{\mathrm{g}}+\mathbf{E}_{\mathrm{vbm}}^{\mathrm{S}}-\chi^{\mathrm{B}}\right)^{3} \\
{ }^{1} B E^{V}\left(\mathrm{Si} 2 \mathrm{p}_{3 / 2}\right)=98.74 \mathrm{eV} \cdot{ }^{2} \mathrm{~h} v=21.2 \mathrm{eV} \cdot{ }^{3} \mathbf{E}_{\mathrm{g}}=1.12 \mathrm{eV} \text { and } \chi^{\mathrm{B}}=4.05 \mathrm{eV}
\end{gathered}
$$




\subsection{Results}

\subsubsection{Surface Characterization}

\subsubsection{XPS Survey Spectra}

The survey spectra of all samples showed peaks at Si 2p (99 eV) and Si 2s (150 $\mathrm{eV}$ ) (Figure 2.3). In addition, $\mathrm{C} 1 \mathrm{~s}(285 \mathrm{eV})$ and $\mathrm{O} 1 \mathrm{~s}(533 \mathrm{eV})$ peaks were observed on all samples, consistent with the presence of adventitious adsorbed hydrocarbons. Using the intensity of the low-energy carbon peak of a $\mathrm{Si}(111)-\mathrm{CH}_{3}$ surface that had little adventitious carbon as a reference for a coverage of one monolayer, the observed carbon and oxygen intensities corresponded to 0.5 - 3 monolayers for various samples. No correlation was observed between the binding energy shifts and the coverage of adventitious species of carbon and/or oxygen. Signals in the $\mathrm{Br} 3 \mathrm{~d}$ region were observed only for $\mathrm{Si}(111)-\mathrm{Br}$ and $\mathrm{Si}(111)-\mathrm{CH}_{3}[\mathrm{Br}]$ surfaces (Figure 2.4). Using the intensity of the Br 3d signal of a $\mathrm{Si}(111)-\mathrm{Br}$ sample as a reference for a coverage of one monolayer, the $\mathrm{Si}(111)-\mathrm{CH}_{3}[\mathrm{Br}]$ surfaces were found to have $0.1-0.3$ monolayers of bromine present on the surface. 


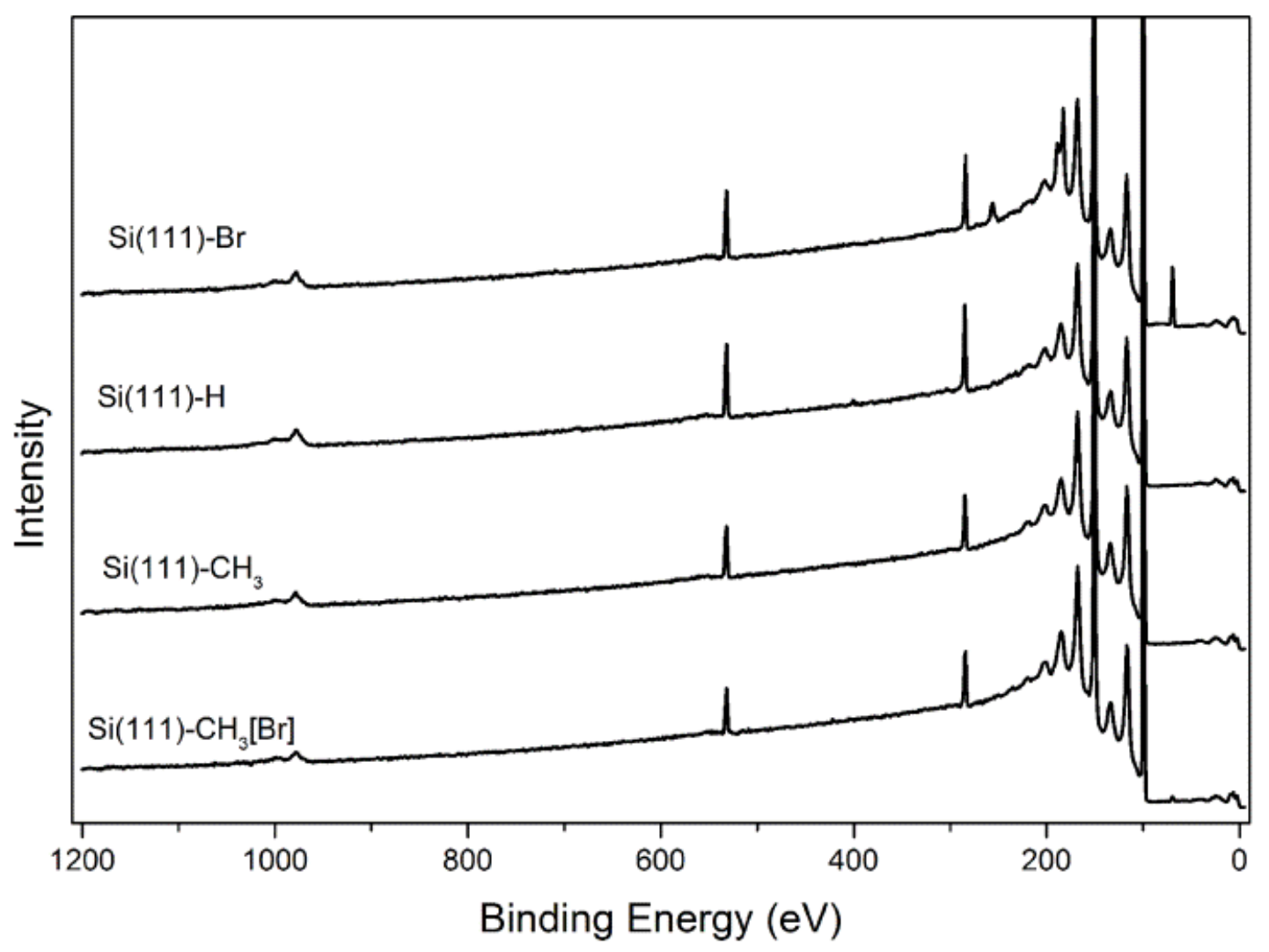

Figure 2.3 XPS Survey Spectra of Si(111) Surfaces. Sample surfaces are clean with adventitious carbon and oxygen accounting for 1-3 monolayers on unannealed surfaces. 


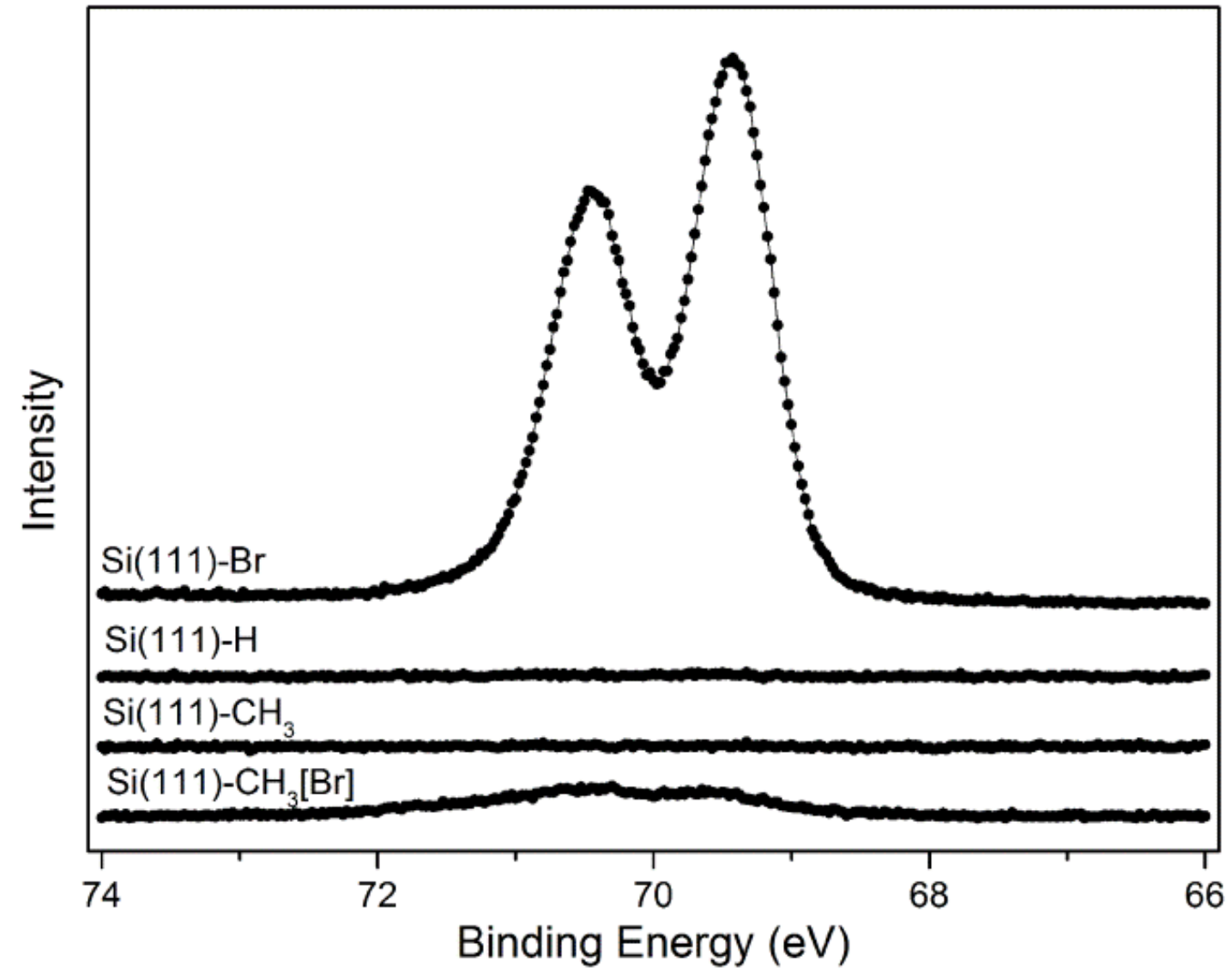

Figure 2.4 XPS Br 3d Spectra of $\mathrm{Si}(111)$ Surfaces. $\mathrm{Si}(111)-\mathrm{CH}_{3}[\mathrm{Br}]$ surface concentration of bromine is $10 \%-30 \%$ (10\% shown) the concentration of $\mathrm{Si}(111)-\mathrm{Br}$ surfaces.

\subsubsection{IR spectra}

The observed FTIR absorbance spectra, with a $\mathrm{Si}(111)-\mathrm{H}$ subtraction (Figures 2.5-2.6), were in good agreement with previously reported data. ${ }^{47}$ No differences were observed between the FTIR spectra of the $\mathrm{Si}(111)-\mathrm{CH}_{3}[\mathrm{Br}]$ and $\mathrm{Si}(111)-\mathrm{CH}_{3}$ surfaces. Both the $\mathrm{Si}(111)-\mathrm{CH}_{3}[\mathrm{Br}]$ and $\mathrm{Si}(111)-\mathrm{CH}_{3}$ surfaces exhibit peaks at $1257 \mathrm{~cm}^{-1}$ (Figure 2.5) which have been ascribed to $\mathrm{C}-\mathrm{H}$ symmetrical bending of the methyl group, known 
as the "umbrella" mode, and which have been demonstrated to be polarized perpendicular to the surface. The $\mathrm{Si}(111)-\mathrm{CH}_{3}[\mathrm{Br}]$ and $\mathrm{Si}(111)-\mathrm{CH}_{3}$ surfaces also have peaks at $757 \mathrm{~cm}^{-1}$ (Figure 2.6) due to the $\mathrm{C}-\mathrm{H}$ rocking motion. ${ }^{47}$

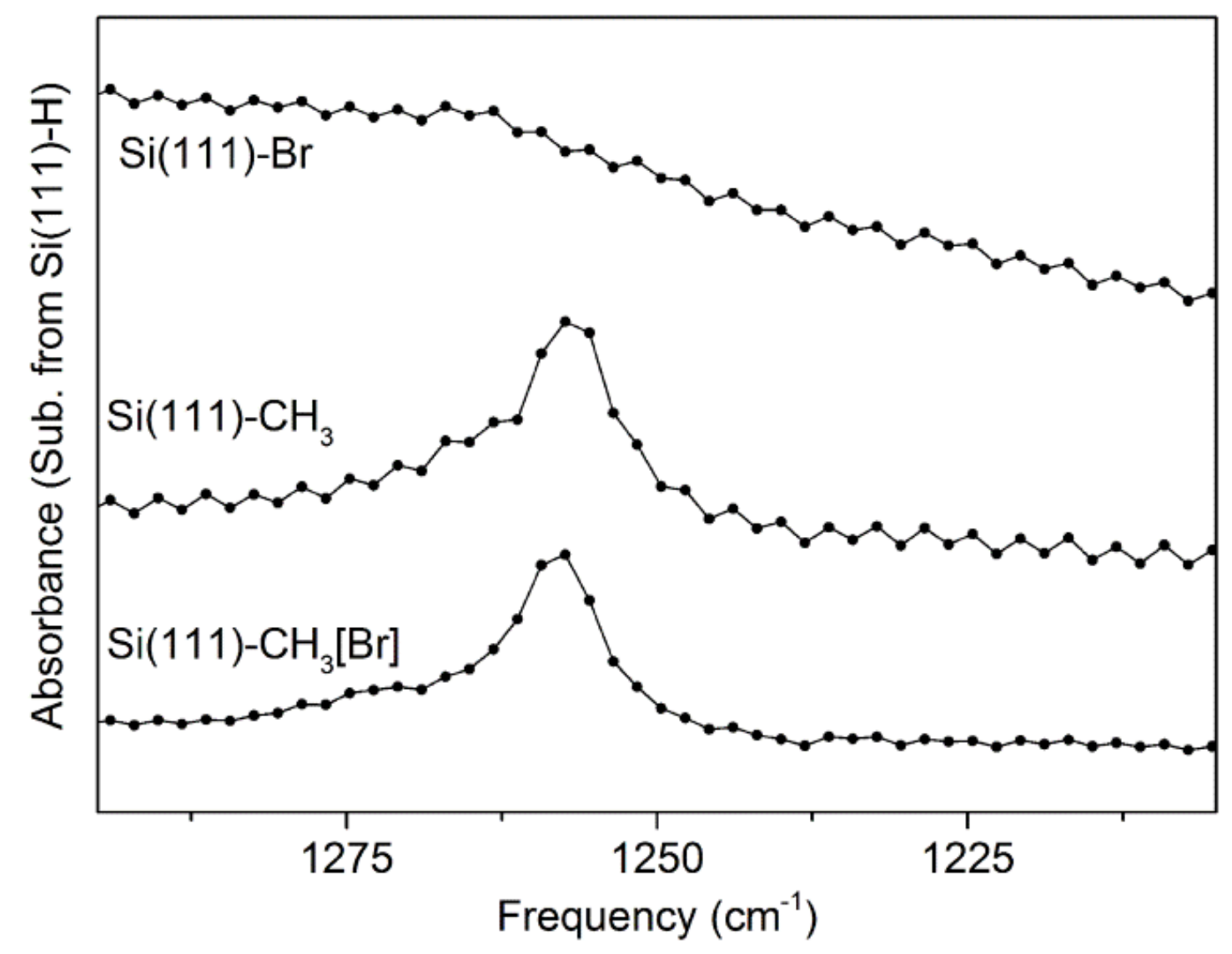

Figure 2.5 IR Absorbance Spectra of Si(111) Surfaces. IR absorbance spectra subtracted from $\mathrm{Si}(111)-\mathrm{H}$ demonstrates no loss of characteristic $\mathrm{Si}(111)-\mathrm{CH}_{3}$ peaks upon exposure to bromine. 


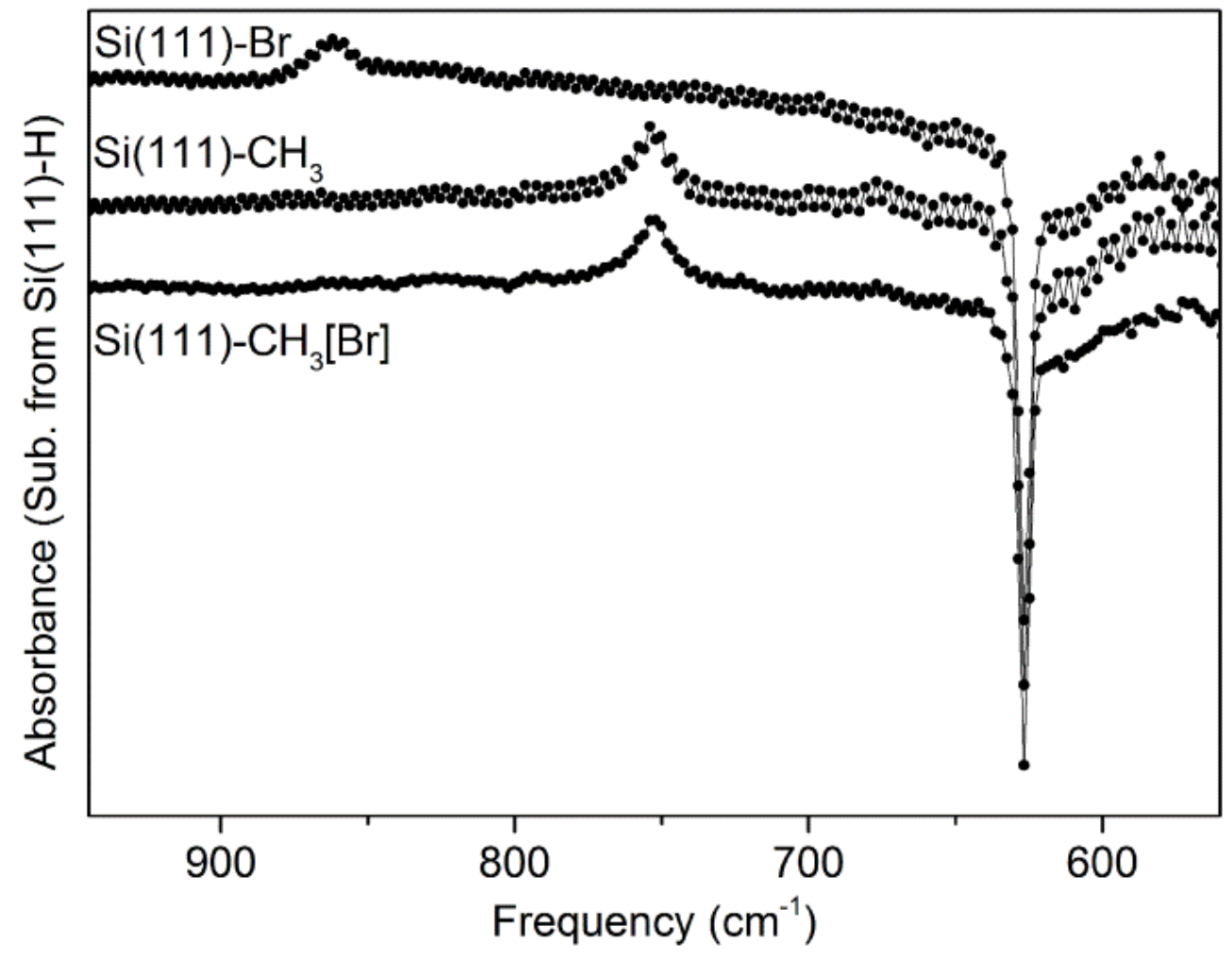

Figure 2.6 IR Absorbance Spectra of Si(111) Surfaces. IR absorbance spectra subtracted from $\mathrm{Si}(111)-\mathrm{H}$ demonstrates no loss of characteristic $\mathrm{Si}(111)-\mathrm{CH}_{3}$ peaks upon exposure to bromine.

\subsubsection{Binding Energy Shifts of Si 2p Peaks in the Dark}

\subsubsection{Moderate Dopant Density}

Figure 2.2a depicts an energy-level diagram of the $\mathrm{Si} 2 \mathrm{p}_{3 / 2}$ binding energy as a function of the observed band bending. Representative spectra are shown in Figure 2.7, with the measurements obtained in the absence of deliberate illumination of the sample depicted in black. The results of all measurements are shown in Figure 2.8 and averages are listed in Table 2.2. 

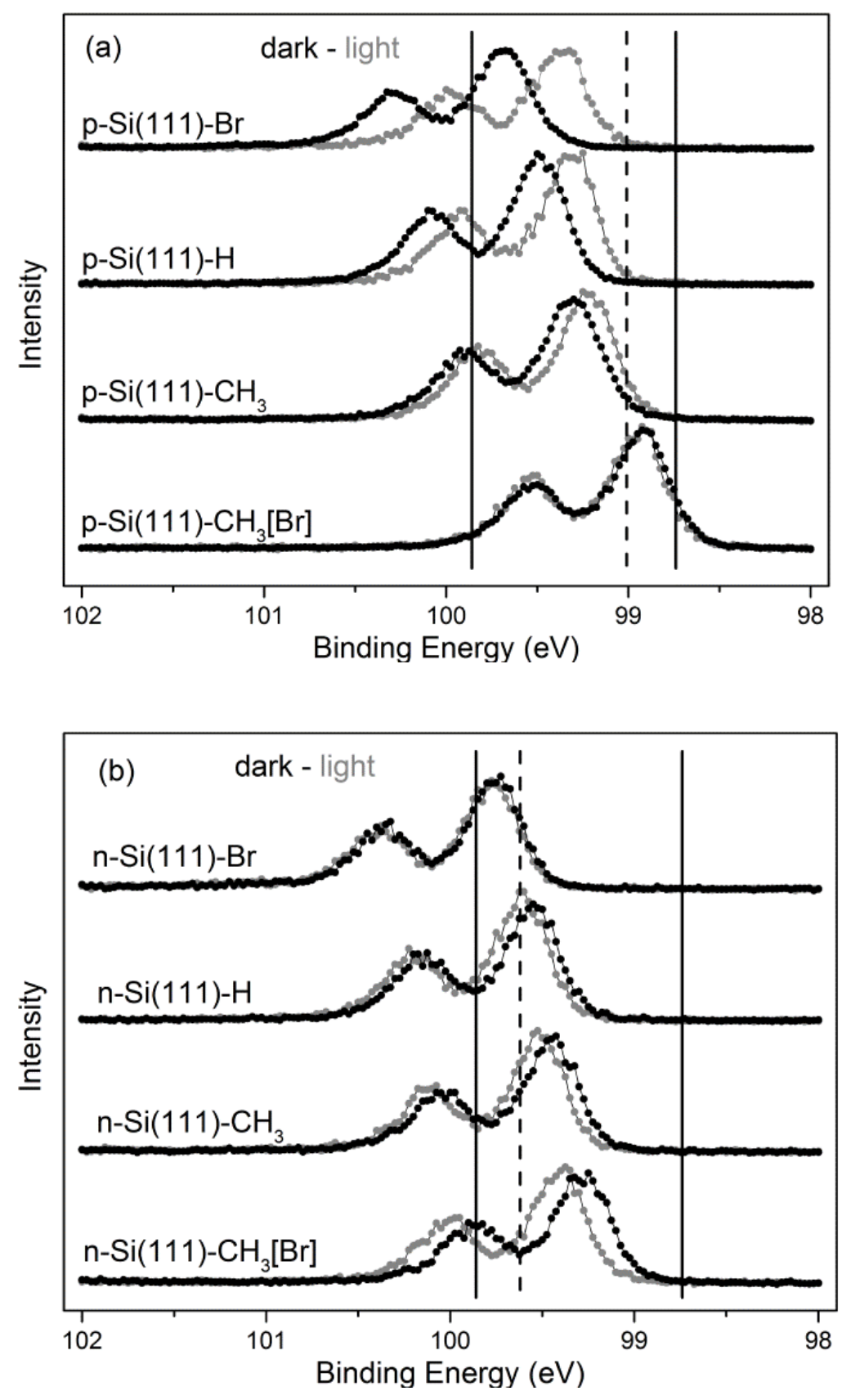

Figure 2.7 XPS Si 2p Spectra of Moderately Doped Si(111) Surfaces. Si 2p core-level spectra of various surfaces on moderately doped (a) p-type and (b) n-type Si (111) using 10 $\mathrm{W}$ X-ray source power. The difference between the conduction band and the Si $2 \mathrm{p}_{3 / 2}$ core level $(99.86 \mathrm{eV})$ and the difference between the valence band and the $\mathrm{Si} 2 \mathrm{p}_{3 / 2}$ core level $(98.74 \mathrm{eV})$ are marked by the solid vertical lines. The difference between the calculated bulk Fermi level and the Si $2 p_{3 / 2}$ core level under flat-band conditions is indicated by the dashed vertical line for each given dopant type. Spectra obtained under deliberate illumination are presented in gray. 


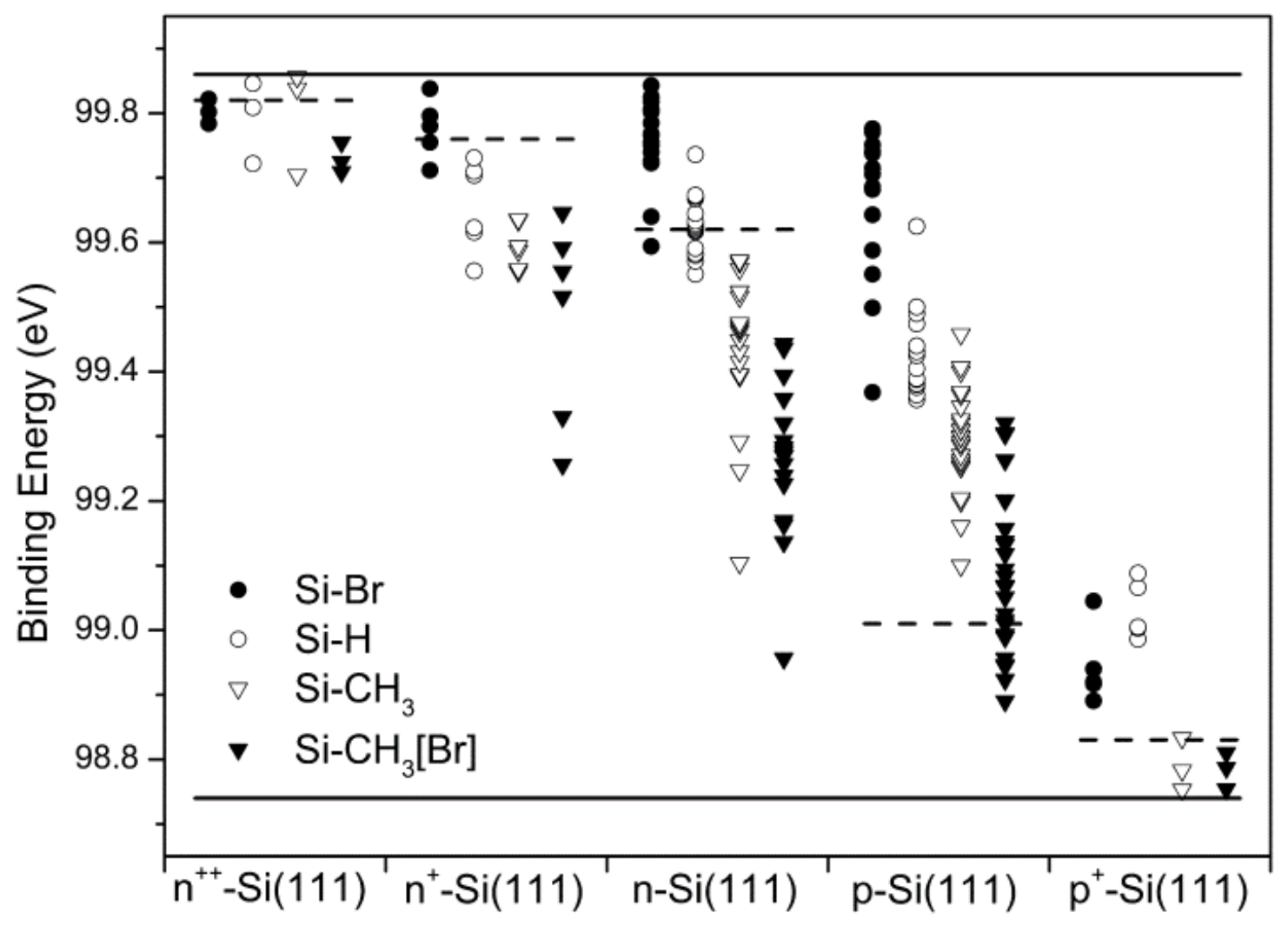

Figure 2.8 Si $2 p_{\mathbf{3} / \mathbf{2}}$ Binding Energy of Functionalized Si Surfaces. Si $2 p_{3 / 2}$ binding energies of all samples using the $10 \mathrm{~W}$ X-ray source power. The solid lines mark the difference between the conduction band and the $\mathrm{Si} 2 \mathrm{p}_{3 / 2}$ core level $(99.86 \mathrm{eV})$ and the difference between the valence band and the $\mathrm{Si} 2 \mathrm{p}_{3 / 2}$ core level $(98.74 \mathrm{eV})$, respectively. The dashed lines mark the difference between the calculated bulk Fermi level and the Si $2 p_{3 / 2}$ core level under flat-band conditions for each dopant density. A significant deviation from flat-band conditions was observed for the moderately doped samples, with surface treatments bending the bands to maintain a specific $\mathbf{E}_{\mathrm{vbm}}^{\mathbf{s}}$ despite different dopant types. 
Table 2.2: Si 2p $3 / 2$ Binding Energy of Functionalized Si Surfaces

\begin{tabular}{|c|c|c|c|c|c|}
\hline Surface & $\begin{array}{l}\text { Samples } \\
(\#)\end{array}$ & $\begin{array}{l}B E\left(\mathrm{Si} 2 \mathrm{p}_{3 / 2}\right) \\
(\mathrm{eV})\end{array}$ & $\begin{array}{l}\sigma^{1} \\
(\mathrm{eV})\end{array}$ & $\begin{array}{l}\mathbf{E}_{\mathrm{vbm}}^{\mathbf{S}} \\
(\mathrm{eV})\end{array}$ & $\begin{array}{l}\mathbf{E}_{\mathrm{bb}}{ }^{2} \\
(\mathrm{eV})\end{array}$ \\
\hline n-Si(111)-Br & 15 & 99.76 & 0.07 & 1.02 & -0.14 \\
\hline n-Si(111)-H & 17 & 99.63 & 0.05 & 0.89 & -0.01 \\
\hline $\mathrm{n}-\mathrm{Si}(111)-\mathrm{CH}_{3}$ & 17 & 99.43 & 0.12 & 0.69 & 0.19 \\
\hline $\mathrm{n}-\mathrm{Si}(111)-\mathrm{CH}_{3}[\mathrm{Br}]$ & 18 & 99.26 & 0.11 & 0.52 & 0.36 \\
\hline $\mathrm{p}-\mathrm{Si}(111)-\mathrm{Br}$ & 15 & 99.66 & 0.12 & 0.92 & -0.65 \\
\hline $\mathrm{p}-\mathrm{Si}(111)-\mathrm{H}$ & 15 & 99.43 & 0.07 & 0.69 & -0.42 \\
\hline $\mathrm{p}-\mathrm{Si}(111)-\mathrm{CH}_{3}$ & 26 & 99.29 & 0.08 & 0.55 & -0.28 \\
\hline $\mathrm{p}-\mathrm{Si}(111)-\mathrm{CH}_{3}[\mathrm{Br}]$ & 24 & 99.08 & 0.13 & 0.34 & -0.07 \\
\hline
\end{tabular}

${ }^{1}$ Standard deviations of the binding energy. ${ }^{2}$ Positive band bending energies indicate that the bands increase in energy as one moves from the bulk to the surface.

For samples with a moderate dopant density, labeled n-Si(111) and p-Si(111), the surface treatment had a larger effect on the binding energy than did the type of dopant. For example, for a given surface functionality, $\mathrm{n}-\mathrm{Si}(111)$ samples exhibited signals that were, on average, $0.15 \mathrm{eV}$ higher in binding energy than the signals observed on $\mathrm{p}$ Si(111) samples. In contrast, different surface treatments produced shifts in the peak binding energies by as much as $0.50 \mathrm{eV}$ on n-type silicon and $0.58 \mathrm{eV}$ on p-type silicon. The trend in binding energy position was consistent for both n-type and p-type Si samples, with $\mathrm{Si}(111)-\mathrm{Br}>\mathrm{Si}(111)-\mathrm{H}>\mathrm{Si}(111)-\mathrm{CH}_{3}>\mathrm{Si}(111)-\mathrm{CH}_{3}[\mathrm{Br}]$. The full width at half-maximum (fwhm) for all peaks was narrow, with values of $0.35 \pm 0.02 \mathrm{eV}$.

\subsubsection{High Dopant Density}

For the highly doped samples, the peak positions were primarily affected by the dopant type, and all signals appeared at energies close to the calculated flat-band 
condition (shown as a dotted line, Figure 2.9). For $\mathrm{n}^{++}-\operatorname{Si}(111)$ samples, the peak positions were in the high range of those observed, while the signals for the $\mathrm{p}^{+}-\operatorname{Si}(111)$ samples were at the low end of the range. For the $n^{+}-\operatorname{Si}(111)$ and $\mathrm{p}^{+}-\operatorname{Si}(111)$ samples, some peak shifting was observed as a result of surface treatment, with the same trend as
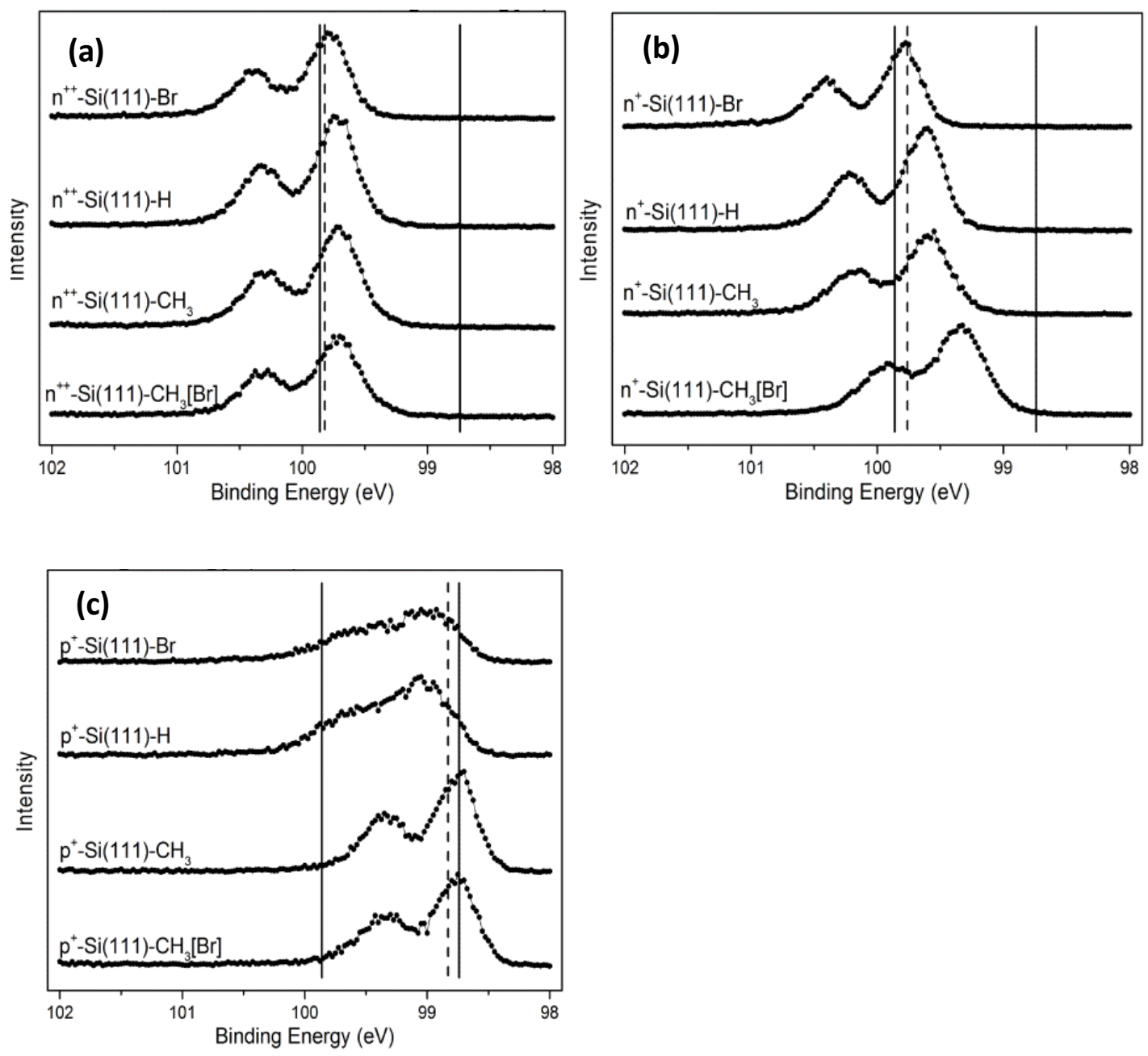

Figure 2.9 XPS Si 2p Spectra of Highly Doped Si(111) Surfaces. Si $2 p$ core-level spectra of various surfaces on (a) very highly doped n-type (b) highly doped n-type and (c) highly doped p-type Si (111) using $10 \mathrm{~W}$ X-ray source power. The difference between the conduction band and the Si $2 p_{3 / 2}$ core level $(99.86 \mathrm{eV})$ and the difference between the valence band and the Si $2 \mathrm{p}_{3 / 2}$ core level $(98.74 \mathrm{eV})$ are marked by the solid vertical lines. The difference between the calculated bulk Fermi level and the Si $2 \mathrm{p}_{3 / 2}$ core level under flat-band conditions is indicated by the dashed vertical line for each given dopant density. 
was observed on the moderately doped Si samples. No significant differences in binding energy were observed as a function of surface treatment on the $\mathrm{n}^{++}-\operatorname{Si}(111)$ surfaces.

In addition to slight peak shifting, some spectra of $\mathrm{n}^{+}-\operatorname{Si}(111)$ and $\mathrm{p}^{+}-\operatorname{Si}(111)$ samples exhibited peak broadening, with an increase in the measured fwhm. The samples that exhibited the broadest peaks were $\mathrm{n}^{+}-\mathrm{Si}(111)-\mathrm{CH}_{3}[\mathrm{Br}], \mathrm{p}^{+}-\mathrm{Si}(111)-\mathrm{Br}$, and $\mathrm{p}^{+}-\mathrm{Si}(111)-\mathrm{H}$ (Figure $2.9 \mathrm{~b}$ and $\left.\mathrm{c}\right)$. The $\mathrm{n}^{++}-\mathrm{Si}(111)$ samples showed no peak broadening (Figure 2.9a).

\subsubsection{Band Bending}

The observed peak shifts from the calculated bulk values (dotted lines), as well as the observed peak broadening, can be explained by the presence of band bending at the silicon/vacuum interface. Band bending changes the binding energy by changing the relative energy from the $\mathrm{Si} 2 \mathrm{p}$ core levels (bent) to the Fermi level (flat). For moderately doped samples, the depletion width is much larger than the escape depth of the electron, so the detected photoelectrons come from a "thin slice" (compared to the full bandbending diagram) of the sample at the silicon/vacuum interface. These detected electrons come from a narrow energy range that is shifted from the flat-band energy by $\mathbf{E}_{\mathrm{bb}}$. 


\subsubsection{Binding Energy Shifts from SPV}

\subsubsection{External Illumination}

Upon illumination, samples furthest from their calculated flat-band energy in the dark showed peaks that shifted significantly toward the calculated flat-band position (Figure 2.7, gray lines). The shifts were approximately half the distance to the calculated flat-band peak position. Conversely, samples that were near the calculated flat-band energy did not show shifts in the peak energies upon illumination. The observed SPV effect is therefore consistent with the interpretation that the binding energy shifts are the result of band bending at the silicon/vacuum interface. The narrow depletion regions of the highly doped samples resulted in no significant peak shifts or peak narrowing upon illumination, due to a lower population in these samples of excited minority carriers that were generated in the depletion region and driven to the surface by drift.

\subsubsection{SPV and X-ray Source Intensity}

The contribution of the X-ray-derived illumination to the SPV was determined by measurement of the $\mathrm{Si} 2 \mathrm{p}_{3 / 2}$ position as a function of the power supplied to the X-ray source (Figure 2.10). For samples that demonstrated some SPV, for example, n-Si(111)$\mathrm{CH}_{3}[\mathrm{Br}]$, increases in the X-ray power source also produced a change in the binding energy position, with a shift toward flat band. No shift was observed for samples that exhibited no SPV, and the shift was not additive with the shift produced by illumination with visible light (i.e., the light trace in Figure 2.10 is flat). As has been suggested previously, ${ }^{16}$ the X-ray source itself contributes to the SPV, so any measurement of band 
bending using XPS therefore needs to carefully consider the intensity of the X-ray source.

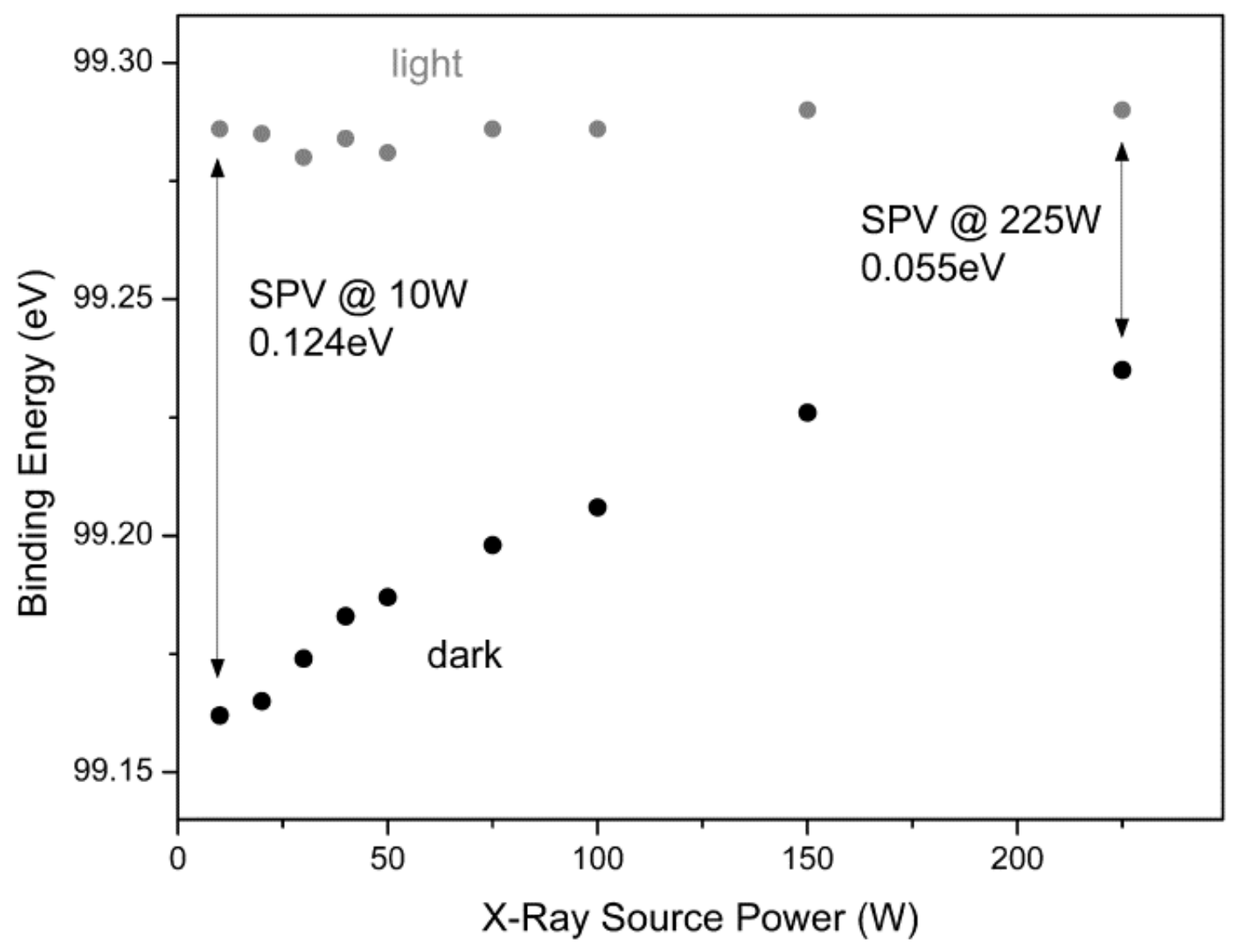

Figure 2.10 Si $2 p_{3 / 2}$ Binding Energies vs X-ray Power. Si $2 p_{3 / 2}$ binding energy as a function of $\mathrm{X}$-ray power for $\mathrm{n}-\mathrm{Si}(111)-\mathrm{Me}[\mathrm{Br}]$ with, and without, external illumination.

Due to very low count rates, peaks other than the Si $2 p$ signal were not well-suited to measurement using $10 \mathrm{~W}$ X-ray emission. Hence, the X-ray source was powered at $150 \mathrm{~W}$, to facilitate comparison between the other spectra that were obtained in this work. Even at higher X-ray intensities, SPV effects were observed clearly in some samples. For example, Figure 2.11 shows the spectra of all regions of interest for a $\mathrm{p}-\mathrm{Si}(111)-\mathrm{Br}$ sample in both the dark (black) and light (gray). The Si $2 p$ region is shown twice, once at 
$10 \mathrm{~W}$ (trace a) and again at $150 \mathrm{~W}$ (trace b). All other XPS spectra (c-f) were collected with the X-ray source set at $150 \mathrm{~W}$. The results were consistent, with all peaks shifting by $\sim-0.4 \mathrm{eV}$ between the light and the dark scans. The Si $2 \mathrm{p}$ peaks observed using 150 $\mathrm{W}$ (trace $\mathrm{b}$ ) exhibited less of a shift in binding energy than those observed using $10 \mathrm{~W}$ of power to the X-ray beam (trace a), due to some SPV being provided by the X-rays themselves at the higher power. The UPS spectra (trace g) exhibited the largest SPVinduced shift in binding energy, indicating that the UV lamp generated less excited carriers in the silicon than the X-ray gun.
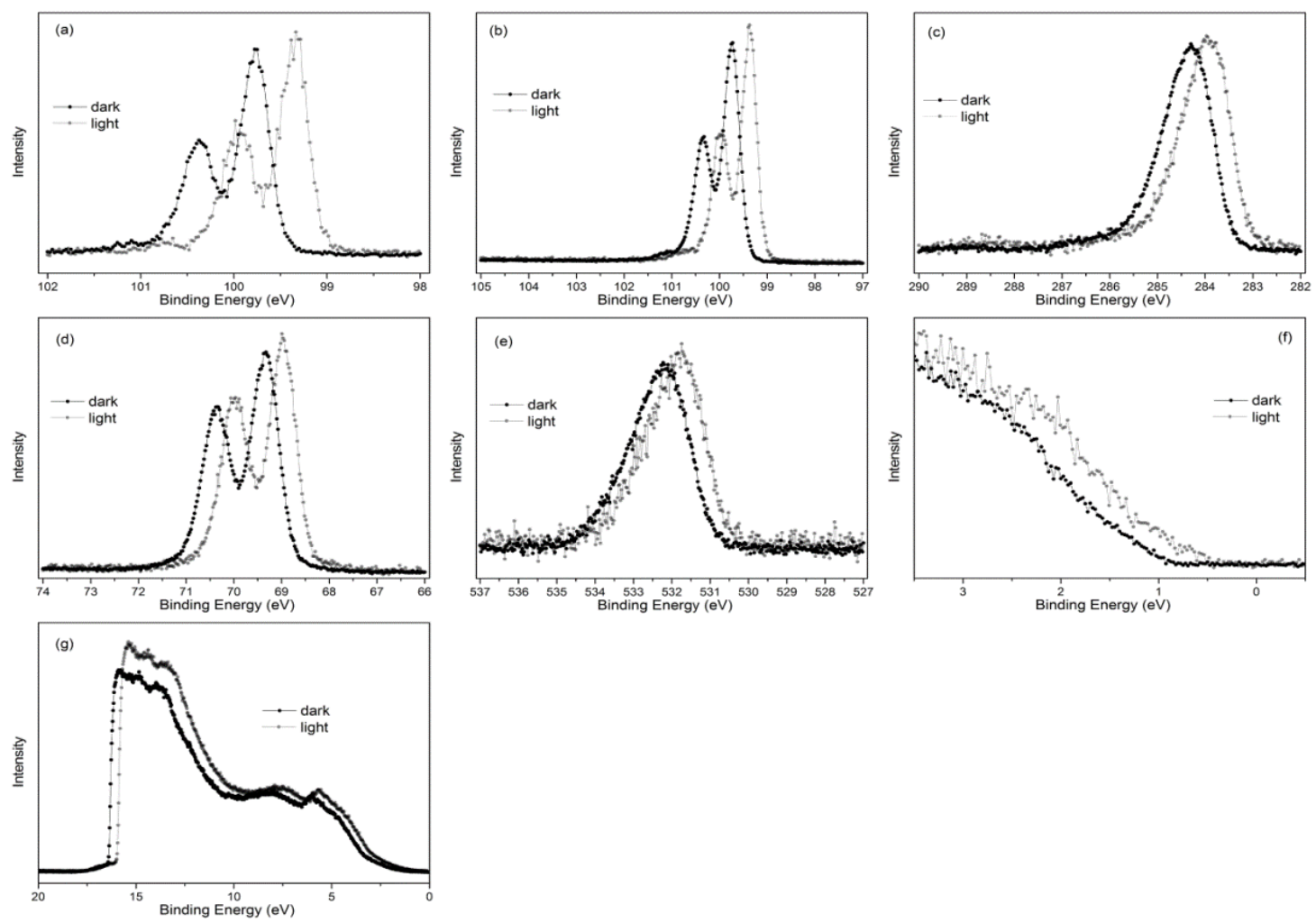

Figure 2.11 Surface Photovoltage Observed by XPS. Surface photovoltage observed on p-Si(111)-Br surface in XPS (a) Si 2p at $10 \mathrm{~W}, \mathrm{SPV}=0.433 \mathrm{eV}$, (b) Si $2 \mathrm{p}$ at $150 \mathrm{~W}, \mathrm{SPV}=$ $0.387 \mathrm{eV}$, (c) C 1s at $150 \mathrm{~W}, \mathrm{SPV}=0.394 \mathrm{eV}$, (d) Br $3 \mathrm{~d}$ at $150 \mathrm{~W}, \mathrm{SPV}=0.390 \mathrm{eV}$, (e) O $1 \mathrm{~s}$ at $150 \mathrm{~W}, \mathrm{SPV}=0.40 \mathrm{eV}$, (f) VBM at $150 \mathrm{~W}, \mathrm{SPV}=0.4 \mathrm{eV}$, and (g) UPS He I spectra, SPV $=0.45 \mathrm{eV}$. 


\subsubsection{UPS Measurements}

\subsubsection{Work Function in Dark}

UPS spectra were obtained both in the light and in the dark, with the secondary electron cutoff region shown in Figure 2.12, and with calculated values listed in Table 2.3. The secondary electron cutoff was measured and was used to calculate the dark work function and the surface dipole. Although the work function is dependent on $\mathbf{E}_{\mathrm{vbm}}^{\mathbf{s}}$, and hence will change with both dopant type and band bending, the surface dipole should be independent of any electronic property or state of the sample. The dark work function demonstrated a clear trend with surface treatment, and very minimal difference due to dopant type, except for the $\mathrm{Si}(111)-\mathrm{CH}_{3}[\mathrm{Br}]$ surface. The smallest work functions were observed for the $\mathrm{Si}(111)-\mathrm{CH}_{3}$ surface; intermediate work functions were observed for the $\mathrm{Si}(111)-\mathrm{H}$ and $\mathrm{Si}(111)-\mathrm{CH}_{3}[\mathrm{Br}]$ surfaces; and the largest work functions were observed for the $\mathrm{Si}(111)-\mathrm{Br}$ surfaces. The work function measurements were not performed with an applied voltage, which is used to ensure that the sample work function is larger than the analyzer work function, due to concerns that an applied voltage could affect the surface band bending. As a result, the smallest work functions measured, at $4.0 \mathrm{eV}$, could be at the threshold of the analyzer work function, and therefore the reported work function for those samples can be interpreted as an upper bound to the sample work function. 

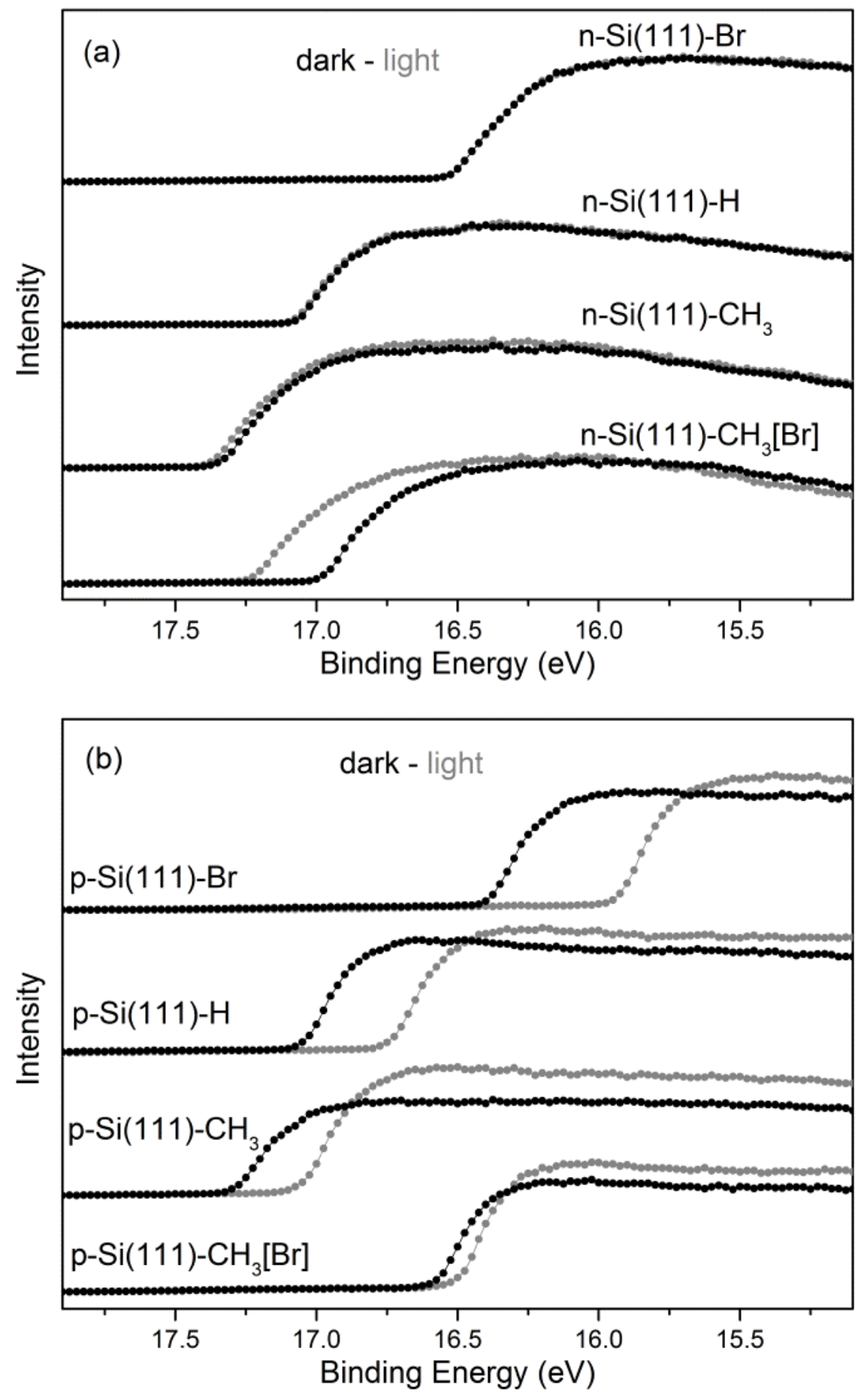

Figure 2.12 UPS He I Spectra. UPS He I spectra of the secondary electron cutoff region for (a) n-type and (b) p-type silicon samples with (gray) and without (black) illumination. 
Table 2.3: Work Function and Surface Dipole of Functionalized Si Surfaces

\begin{tabular}{lllll}
\hline Surface & $\begin{array}{l}W_{\mathrm{F}, \mathrm{s}} \text { dark } \\
(\mathrm{eV})\end{array}$ & $\begin{array}{l}W_{\mathrm{F}, \mathrm{s}} \text { light } \\
(\mathrm{eV})\end{array}$ & $\begin{array}{l}\delta \text { dark } \\
(\mathrm{eV})\end{array}$ & $\begin{array}{l}\delta \text { light } \\
(\mathrm{eV})\end{array}$ \\
\hline $\mathrm{n}-\mathrm{Si}(111)-\mathrm{Br}$ & 4.86 & 4.86 & 0.71 & 0.73 \\
n-Si(111)-H & 4.29 & 4.27 & -0.07 & -0.03 \\
n-Si(111)-CH & 4.04 & 4.01 & -0.42 & -0.38 \\
n-Si(111)-CH$[\mathrm{Br}]$ & 4.40 & 4.17 & -0.23 & -0.33 \\
\hline p-Si(111)-Br & 4.94 & 5.39 & 0.80 & 0.82 \\
p-Si(111)-H & 4.28 & 4.59 & -0.27 & -0.08 \\
p-Si(111)-CH & 4.05 & 4.28 & -0.50 & -0.40 \\
p-Si(111)-CH $\mathrm{CH}_{3}[\mathrm{Br}]$ & 4.72 & 4.82 & 0.01 & 0.00 \\
\hline
\end{tabular}

\subsubsection{Work Function under Illumination}

The work functions of illuminated samples were measured and compared to the dark work functions, as shown in Figure 2.12 (gray lines) and in Table 2.3. For the ptype samples, illumination increased the difference between the work functions of the different surface types, in stark contrast to the Si $2 p$ XPS peak positions, which were more similar to each other after illumination. Both results conform to the band-bending analysis depicted in Figure 2.2.

\subsubsection{Binding Energy Shifts of C 1s Peaks}

Figure 2.13 shows representative measurements of the binding energy of the $\mathrm{C} 1 \mathrm{~s}$ peak relative to the binding energy of the $\mathrm{Si} 2 \mathrm{p}_{3 / 2}$ peak (at $150 \mathrm{~W}$ ), with both measurements performed on the same sample. The difference in peak positions was used to eliminate effects due to differences in band bending, because both peaks are equally affected by band bending, as demonstrated by the SPV effect shown in Figure 2.11b-c. 


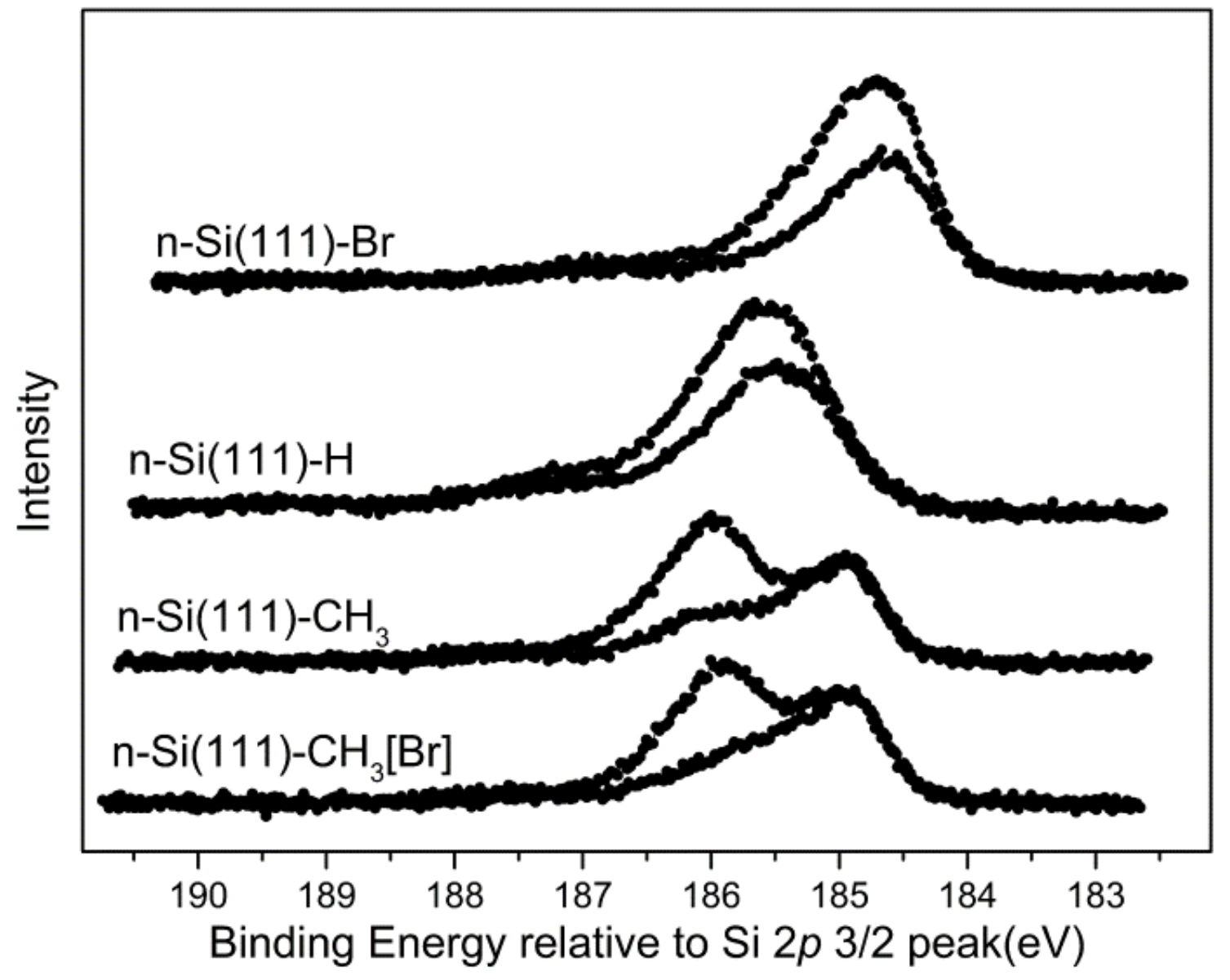

Figure 2.13 XPS C 1s Spectra. XPS of the C 1s region relative to the Si $2 p_{3 / 2}$ binding energy position of the same sample. The two overlaid spectra are of the same sample after an elapsed time of $47.5 \mathrm{hrs}$. The difference is the result of adventitious carbon adsorbed in the vacuum chamber. The subtraction from the Si $2 p$ peak enables isolation of the shift caused by the dipole rather than the shift due to band bending.

The spectra exhibited signals due to adventitious carbon ( $\mathrm{C}-\mathrm{C}$ bonds and $\mathrm{C}-\mathrm{H}$ bonds), and spectra of the $\mathrm{Si}(111)-\mathrm{CH}_{3}$ and $\mathrm{Si}(111)-\mathrm{CH}_{3}[\mathrm{Br}]$ surfaces also exhibited peaks due to silicon-bound carbon. On the $\mathrm{Si}(111)-\mathrm{CH}_{3}$ and $\mathrm{Si}(111)-\mathrm{CH}_{3}[\mathrm{Br}]$ surfaces, the carbon bound to silicon was downshifted by $\sim 1 \mathrm{eV}$ relative to the adventitious carbon signal. A much higher binding energy shoulder, upshifted from the $\mathrm{C}-\mathrm{C} / \mathrm{C}-\mathrm{H}$ adventitious carbon 
signal by $\sim 1.5 \mathrm{eV}$, resulting from carbon bound to oxygen, was also observed on all samples. As shown in Figure 2.13, the location of the adventitious carbon signal was variable and depended on the surface treatment. The relative binding energy position of the adventitious carbon on $\mathrm{Si}(111)-\mathrm{Br}$ was the lowest measured, and the position of the adventitious carbon on $\mathrm{Si}(111)-\mathrm{CH}_{3}$ was the highest measured, with a difference of 1.0 $\mathrm{eV}$ between them. These shifts are consistent with the adventitious carbon being located exterior to the surface dipole and being affected by the value of the surface dipole, as depicted in Figure 2.2b.

To ensure that the observed shifts were not the result of chemically different carbonaceous species present on the different surfaces, the signals produced by adventitious carbon that had been deposited in vacuum were also recorded. Without any intentional introduction of carbon, the base adventitious carbon deposition rate on samples in the UHV chamber was found to be 0.027 monolayers per hour, using the intensity of the silicon-bound carbon (C-Si) signal on a $\mathrm{Si}(111)-\mathrm{CH}_{3}$ surface as a reference for 1.0 monolayer of coverage. After two days of adventitious carbon growth, which was performed simultaneously for all four samples used in the measurement, the peaks for adventitious carbon deposited in the vacuum chamber lined up with peaks previously ascribed to adventitious carbon resulting from atmospheric exposure. No significant chemical difference was assumed between the adventitious carbon deposited on one sample as compared to another, and so shifts in the binding energy position cannot be ascribed to chemical changes. Analysis of Figure 2.13 indicates some slight asymmetry in the adventitious growth $\mathrm{C}$ on the $\mathrm{Si}(111)-\mathrm{Br}$ and $\mathrm{Si}(111)-\mathrm{H}$ samples, 
consistent with a decrease in the dipole strength with increased distance, due to a small induced dipole in the opposite direction on the interior adventitious carbon layers. The initial surface concentrations of adventitious carbon were not constant, with initial scans of $\mathrm{Si}(111)-\mathrm{CH}_{3}$ and $\mathrm{Si}(111)-\mathrm{CH}_{3}[\mathrm{Br}]$ showing less than 0.5 monolayers of adventitious carbon, while $\mathrm{Si}(111)-\mathrm{Br}$ and $\mathrm{Si}(111)-\mathrm{H}$ surfaces had over 2 monolayers of adventitious carbon. The low levels of adventitious carbon on the $\mathrm{Si}(111)-\mathrm{CH}_{3}$ surfaces were achieved without annealing and in spite of the introduction of such samples to the vacuum chamber through ambient air.

\subsection{Discussion}

\subsubsection{Band Bending and $Q_{s s}$}

\subsubsection{Moderate Dopant Density}

Functionalization of $\mathrm{Si}(111)$ surfaces has been demonstrated to induce band bending such that any given surface will tend toward some specific value of $\mathbf{E}_{\mathrm{vbm}}^{\mathbf{s}}$ regardless of the dopant type of the silicon. Samples with large positive dipoles, namely Si(111)-Br surfaces, resulted in large values for $\mathbf{E}_{\mathbf{v b m}}^{\mathbf{s}}$, with the Fermi level positioned near the conduction band for both n-type and p-type samples. The p-type samples exhibited significant band bending, while the n-type samples were near flat-band, as indicated by the SPV measurements. For $\mathrm{Si}(111)-\mathrm{H}$ samples, with a surface dipole near zero, the measured $\mathbf{E}_{\mathrm{vbm}}^{\mathbf{S}}$ was consistently lower than the measured $\mathbf{E}_{\mathrm{vbm}}^{\mathbf{S}}$ of the $\operatorname{Si}(111)$ - 
Br surfaces. $\mathrm{Si}(111)-\mathrm{CH}_{3}$ samples had a negative surface dipole and exhibited a Fermi level position that was near the middle of the band gap.

Band bending at the silicon/vacuum interface is the result of charged surface states, with the net surface charge density, $Q_{s s}$, equal and opposite to the net surface charge density for the space-charge region, $Q_{\text {sc }}$, given by the product of the dopant density and the depletion width, ${ }^{1}$

$$
\left|Q_{\mathbf{s c}}\right|=e N_{\mathbf{D}} W=\sqrt{2 e \varepsilon_{\mathbf{S i}} N_{\mathbf{D}}\left(\frac{\left|\mathbf{E}_{\mathrm{bb}}\right|-k T}{e}\right)} .
$$

where $e$ is the charge on a proton, $\varepsilon_{S i}$ is the permittivity of silicon $\left(11.8^{*} \varepsilon_{0}\right), N_{\mathrm{D}}$ is the dopant density, $\mathbf{E}_{\mathrm{bb}}$ is the band bending energy, $k$ is the Boltzmann constant, $T$ is the absolute temperature $\left(k T\right.$ is $0.0256 \mathrm{eV}$ at $\left.25^{\circ} \mathrm{C}\right)$, and $W$ is the width of the space-charge region. For moderately doped silicon with a high degree of band bending, $Q_{\text {ss }}$ is in the range of 1-3 $\times 10^{-8} \mathrm{C} \mathrm{cm}^{-2}$, i.e., 1-3 $\times 10^{-4}$ charged surface atoms per surface atom. Note that while the space-charge region is a volume, the space-charge density, $Q_{s c}$, is a charge per unit area.

\subsubsection{High Dopant Density}

For highly doped samples, the band bending can give rise to peak broadening if the depletion region is comparable in width to the escape depth of the electron. The collected photoelectrons would then have a range of energies that corresponds to the band bending. The lack of any observed peak shifting or broadening for the $\mathrm{n}^{++}-\operatorname{Si}(111)$ 
samples (Figure 2.8a) suggests that there is a very narrow depletion width, so that for all surface treatments, the majority of collected electrons came from the flat-band region. The calculated depletion width is $8 \mathrm{~nm}$, but possible surface gettering of dopants could reduce it. Also, small discrepancies at very low resistivity give rise to significant changes in the calculated width.

The observed $\mathrm{BE}\left(\mathrm{Si} 2 \mathrm{p}_{3 / 2}\right)$ for $\mathrm{n}^{++}-\mathrm{Si}(111)$ samples were independent of surface treatment, confirming that they are not affected by the surface dipole. The high dopantdensity samples provide qualitative support for understanding the observed binding energy shifts in terms of band bending; however, using this technique, high dopantdensity samples cannot provide quantitative values for $\mathbf{E}_{\mathrm{vbm}}^{\mathrm{s}}$ or $Q_{s s}$.

\subsubsection{Surface Dipole and $Q_{s s}$}

\subsubsection{Electronegativity}

The trend in $W_{\mathrm{F}, \mathrm{S}}$ (Figure 2.12 and Table 2.3) is the opposite of that which would be expected if $W_{\mathrm{F}, \mathrm{s}}$ is only affected by the band bending. As seen in Figure $2.2 \mathrm{~b}$, surface band bending that causes the Fermi level to be at or near the conduction band, as is the case with the $\operatorname{Si}(111)-\mathrm{Br}$ surface $\left(\mathbf{E}_{\mathrm{vbm}}^{\mathrm{s}} \sim 1 \mathrm{eV}\right)$, should result in low $W_{\mathrm{F}, \mathrm{s}}$. However, these samples have the largest work functions (Table 2.3). The effect of the surface dipole must therefore be large and in the opposite direction to the band bending.

The measured dipoles, shown in Table 2.2, are in good agreement with previously reported surface dipoles on similar surfaces as well as in accord with considerations 
based on differences in the Pauling electronegativities of the various functionalities on the surface, with values of $1.90,2.20,2.55$, and 2.96 for $\mathrm{Si}, \mathrm{H}, \mathrm{C}$, and $\mathrm{Br}$, respectively. The large positive $\delta$ (positive referring to the energy shift on a band energy diagram as one moves from the sample to the vacuum) for the $\mathrm{Si}(111)-\mathrm{Br}$ surfaces can be explained in terms of the electron-withdrawing characteristics of the bromine atom creating a significant charge separation, represented as $\mathrm{Si}^{{ }^{+}}-\mathrm{Br}^{\delta_{-}^{-}}$. The smaller electronegativity difference between the $\mathrm{Si}$ and $\mathrm{H}$ is consistent with the small dipole observed for the $\mathrm{Si}(111)-\mathrm{H}$ surfaces. Consideration of the electronegativity difference between the $\mathrm{Si}$ and $\mathrm{C}$ would lead to a positive dipole of intermediate magnitude, but previous work ${ }^{30}$ has demonstrated that the $\mathrm{C}^{\delta-}-\mathrm{H}^{\delta+}$ bond plays a more important role in determining the surface dipole than the $\mathrm{Si}^{\delta+}-\mathrm{C}^{\delta-}$ bond. The measured dipoles for the $\mathrm{Si}(111)-\mathrm{CH}_{3}$ surfaces therefore reflect the low values of the electron density at these surfaces.

Bromine termination of the Si surfaces resulted in either little net surface charge (small $\mathbf{E}_{\mathrm{bb}}$ ) for n-type samples or in a relatively large amount of net positive charge (large negative $\mathbf{E}_{\mathrm{bb}}$ ) for p-type samples. By definition, the surface dipole contributes no net charge, but charged states near the dipole could be induced and/or stabilized. Silicon bound to bromine will have a partial positive charge reflected in the work function. The electron-withdrawing effects of the bromine would destabilize cation formation on the attached silicon, and to a lesser degree, any of the nearest neighbors to the silicon. Conversely, any positive charge on the vacuum side of the $\mathrm{Si}-\mathrm{Br}$ bond would be stabilized by the electron density on the bromine. 


\subsubsection{Energy-Level Diagram}

An alternative framework would be to consider the band-level diagram shown in Figure 2.14. A surface dipole will move all levels of a species located outside the dipole layer relative to the Fermi level of the surface. Thus a positive $\delta$ moves outside levels toward the vacuum level, while a negative $\delta$ will move the outside levels toward lower energy. This shift is observed for the adventitious C 1s peaks. As shown in Figure 2.13, the $\mathrm{C} 1 \mathrm{~s}$ peak shifted by 0.8 and $1.1 \mathrm{eV}$, respectively, when the surface termination was changed from $\mathrm{Si}-\mathrm{Br}$ to $\mathrm{Si}-\mathrm{H}$ and from $\mathrm{Si}-\mathrm{Br}$ to $\mathrm{Si}_{-} \mathrm{CH}_{3}$ surfaces. The energy difference of $\delta$ on the same surfaces is 0.8 and $1.3 \mathrm{eV}$, respectively. Thus the adventitious carbon peaks shifted in accord with expectations for a species located outside of the dipole layer. If the Fermi level of the semiconductor equilibrates with a species located outside of a surface dipole layer, then the observed $\delta$ will correlate with $\mathbf{E}_{\mathbf{v b m}}^{\mathbf{s}}$ and will inversely correlate with $\mathbf{E}_{\mathrm{bb}}$.

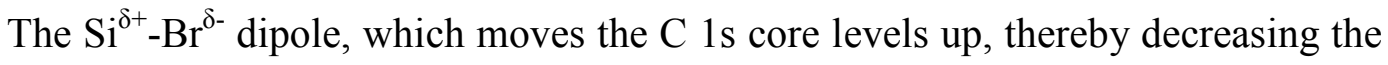
distance from the core level to Fermi level, will also shift any energy levels at the Fermi level to a position above the Fermi level (Figure 2.14b). Filled states above the Fermi level will then donate their electrons to the semiconductor, and if such states were neutral they will then become positively charged. This process will continue until all of the filled donors lose their electrons or the energy level of the redox couple equilibrates with the Fermi level. In this model, a positive dipole would be expected to produce downward band bending (Figure 2.14c). 
(a)

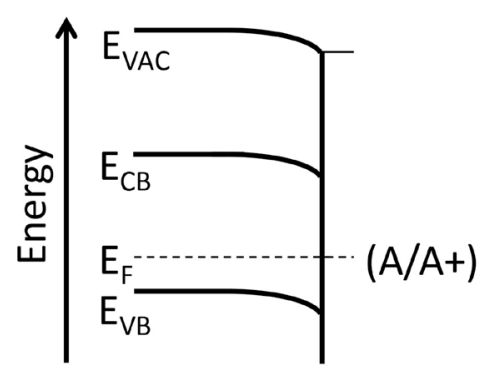

(d)

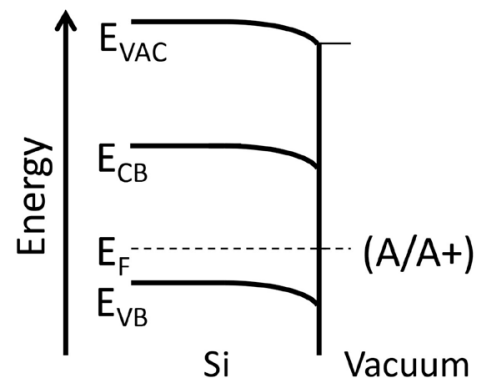

(b)

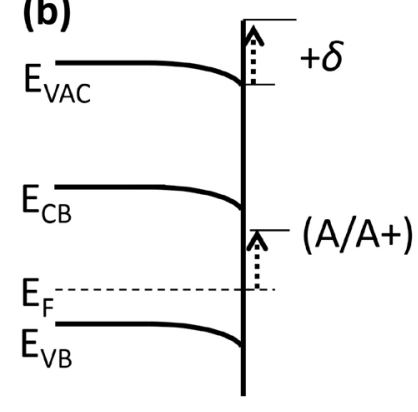

(e)

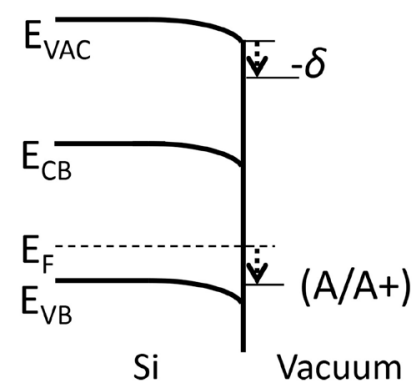

(c)

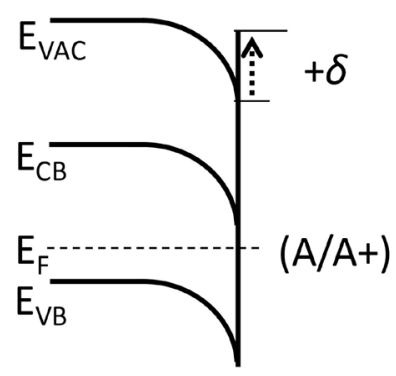

(f)

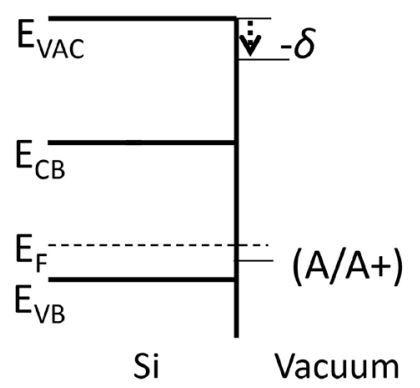

Figure 2.14 Band-Level Diagrams of Model System. Band-level diagrams of a model system in which the surface charge is a redox species located exterior to the dipole. Starting conditions (a) and (d) depict no dipole, and the redox species equilibrated to the Fermi level of the semiconductor resulting in a small amount of band bending. Introduction of a dipole, but prior to any electron transfer, will cause the redox species to be shifted up relative to the Fermi level for positive dipoles (b) and down for negative dipoles (e). Redox species above the Fermi level will donate electrons to the semiconductor resulting in more downward band bending(c), while those below the Fermi level will accept electrons causing the bands to flatten (f). This model could be expanded by adding counterions to achieve positive band bending.

Within this framework, for a negative dipole, such as the $\mathrm{C}^{\delta-}-\mathrm{H}^{\delta+}$ bond on a $\mathrm{Si}(111)-\mathrm{CH}_{3}$ surface, all of the energy levels of the adsorbed molecules should be shifted downward. This shift will increase the observed core-level binding energy of adventitious carbon and will move any energy levels at the Fermi level to a position below the Fermi level (Figure 2.14d-e). Any unfilled acceptors, in this case cations, would be filled, decreasing the net surface charge and reducing the band bending. As 
shown in Figure 2.14f, all of the acceptor states have been filled, and so such states will no longer equilibrate with the Fermi level. The partial positive charge on the hydrogen atoms would destabilize any adjacent cations.

\subsubsection{Surface Dipole vs. Terminal Si Electron Density}

The inverse relationship between the surface dipole and the observed band bending is not related to the electron density on the terminal silicon atom. Although the

$\mathrm{C}^{\delta-}-\mathrm{H}^{\delta+}$ bond results in a negative dipole, the $\mathrm{Si}^{{ }^{+}}-\mathrm{C}^{\delta-}$ bond is more polar than the $\mathrm{Si}^{\delta+}-\mathrm{H}^{\delta-}$ bond. This behavior would place the $\mathrm{Si}(111)-\mathrm{CH}_{3}$ surface between the $\mathrm{Si}(111)-\mathrm{Br}$ surface (strongly polar) and $\mathrm{Si}(111)-\mathrm{H}$ surface (weakly polar) in terms of electron density on the bonded silicon atom. Therefore, no trend was observed in the band bending as a function of the chemical bond polarity of the terminal silicon atom.

\subsubsection{Redox Adsorption}

The very low $\mathbf{E}_{\mathrm{vbm}}^{\mathrm{s}}$ values observed for $\mathrm{Si}(111)-\mathrm{CH}_{3}[\mathrm{Br}]$ samples do not neatly fit the trend in surface dipoles but still fit within the framework of the larger model advanced herein. The total elemental bromine on the $\mathrm{Si}(111)-\mathrm{CH}_{3}[\mathrm{Br}]$ surface was only $10-30 \%$ of the total bromine on the $\mathrm{Si}(111)-\mathrm{Br}$ samples, and no chemical bond changes were observed by IR (Figure 2.5-2.6) or XPS analysis (Figure 2.3 and 2.7). As seen in Figure 2.13, no appreciable loss of silicon-bound carbon was observed in the $\mathrm{C} 1 \mathrm{~s}$ spectra, and no signals ascribable to $\mathrm{C}-\mathrm{Br}$ bonds were observed. The $\mathrm{Br} 3 \mathrm{~d}$ XPS spectra, Figure 2.4, indicate the presence of multiple oxidation states of $\mathrm{Br}$ on such surfaces, but 
soft-XPS would be better suited to elucidate the nature of the bromine on the surface. Possible states of bromine include $\mathrm{Br}_{2}, \mathrm{Br}^{-}, \mathrm{Br}_{3}^{-}, \mathrm{BrO}^{-}$, and $\mathrm{BrO}_{3}^{-}$. Fixing the surface Fermi level near the valence band requires either no net surface charge for p-type samples or net negative charge for n-type samples. If a small amount of the bromine on the surface were present as an anion (without a counterion), a low $\mathbf{E}_{\mathrm{vbm}}^{\mathbf{s}}$ would be expected.

The traditional model of surface states locates the charged atom on the semiconductor side of the surface dipole. The rationale for this approach comes from the long history of semiconductor metal contacts, in which the excess charge could not be located on the metal. Defect sites, dangling bonds, step edges, and impurity atoms (at a much higher concentration than dopant atoms) have been suggested as possible locations for these charges. The model advanced herein locates the surface charge exterior to the surface dipole on adsorbed redox species. The redox species could be introduced either in the gas phase (even in vacuum) or during the solvent rinses. Any adsorbed species that formed an "ion pair" with the semiconductor would be difficult to remove upon introduction to vacuum, due to the electrostatic attraction. Introduction to vacuum would remove solvating molecules, and so would have an effect on the redox potential of the species. Adsorbed water should be considered as a possible source of surface charge, considering the common ionization of water in the generation of static charge. Furthermore, the role of surface chemistry and the surface dipole has long been known to stabilize/destabilize the hydronium and hydroxide ions in the generation of static charge, known as the triboelectric effect. Gas-phase adsorbed water has been demonstrated to 
affect the band bending similar to an adsorbed redox couple, ${ }^{48}$ and humidity affects the surface conductivity of silicon. ${ }^{49-50}$

\subsubsection{Measurement Variability}

The relatively large range of values observed for the $\mathrm{Si} 2 \mathrm{p}_{3 / 2}$ peak position on moderately doped samples indicates the need for a statistical analysis of band-bending measurements. Measurement variability, even on a single sample measured at different times left in vacuum, demonstrates the need for data collection on a large number of samples. The band bending of a semiconductor in vacuum is not an inherent property of a semiconductor; rather, it is influenced by the surface chemistry, adsorbed species, and sample history. The variability is consistent with adsorbed ions/redox couples giving rise to band bending. Molecular adsorption has been demonstrated to take place in vacuum for adventitious carbon, and trace adsorption is difficult to rule out in standard experimental measurements.

The Si $2 p$ XPS spectra were used to determine the position of the Fermi level at the surface, $\mathbf{E}_{\mathrm{vbm}}^{\mathbf{s}}$, whereas the UPS spectra were used to determine the work function, $W_{\mathrm{F}, \mathrm{s}}$. Derivation of the surface dipole by this approach thus assumed that the same band bending was present in both cases. However, even at very low power, the XPS clearly generated some SPV. For this reason, determination of $\mathbf{E}_{\mathrm{vbm}}^{\mathbf{s}}$ and $W_{\mathrm{F}, \mathrm{s}}$ in the light may give a more accurate measurement of the surface dipole than measurements of the dipole performed in the dark. 


\subsection{Conclusions}

XPS studies have demonstrated variable band bending on chemically modified Si(111) surfaces, with the surface dipole strongly correlated with $\mathbf{E}_{\mathbf{v b m}}^{\mathbf{S}}$ at the surface. Si(111)-Br surfaces, with large positive dipoles, exhibited either no net surface charge (ntype) or a relatively large amount of net positive surface charge (p-type). $\mathrm{Si}(111)-\mathrm{CH}_{3}$ surfaces, with negative surface dipoles, exhibited either net negative charge (n-type) or net positive charge (p-type). The band bending on such surfaces was confirmed by SPV, wherein the bending of the bands was reduced upon illumination. The X-ray source was found to contribute to charge-carrier generation, but this effect was mitigated by the use of a low-power setting for the X-ray beam. The binding energy positions of adsorbed adventitious carbon shifted due to $\mathbf{E}_{\mathbf{v b m}}^{\mathbf{S}}$ as well as due to the presence of any surface dipoles. Evidence of dipole-induced energy shifts of adsorbed molecules are consistent with a model in which the charged surface states are adsorbed redox couples that are located on the vacuum side of the dipole. 


\subsection{References}

(1) Sze, S. M. Physics of Semiconductor Devices; Wiley: New York, 1981.

(2) Cowley, A. M.; Sze, S. M. Surface States and Barrier Height of MetalSemiconductor Systems. J. Appl. Phys. 1965, 36 (10), 3212-3220.

(3) Zhang, Z.; Yates, J. T., Jr. Band Bending in Semiconductors: Chemical and Physical Consequences at Surfaces and Interfaces. Chem. Rev. 2012, 112 (10), 5520-51.

(4) Bardeen, J. Surface States and Rectification at a Metal Semi-Conductor Contact. Phys. Rev. 1947, 71 (10), 717-727.

(5) Bocarsly, A. B.; Bookbinder, D. C.; Dominey, R. N.; Lewis, N. S.; Wrighton, M. S. Photo-Reduction at Illuminated P-Type Semiconducting Silicon Photoelectrodes Evidence for Fermi Level Pinning. J. Am. Chem. Soc. 1980, 102 (11), 3683-3688.

(6) Bard, A. J.; Bocarsly, A. B.; Fan, F. R. F.; Walton, E. G.; Wrighton, M. S. The Concept of Fermi Level Pinning at Semiconductor-Liquid Junctions Consequences for Energy-Conversion Efficiency and Selection of Useful Solution Redox Couples in Solar Devices. J. Am. Chem. Soc. 1980, 102 (11), 3671-3677.

(7) Lewerenz, H. J. Surface-States and Fermi-Level Pinning at Semiconductor Electrolyte Junctions. J. Electroanal. Chem. 1993, 356 (1-2), 121-143.

(8) Dominey, R. N.; Lewis, N. S.; Bruce, J. A.; Bookbinder, D. C.; Wrighton, M. S. Improvement of Photo-Electrochemical Hydrogen Generation by Surface Modification of P-Type Silicon Semiconductor Photo-Cathodes. J. Am. Chem. Soc. 1982, 104 (2), 467-482.

(9) Sharma, J.; Staley, R. H.; Rimstidt, J. D.; Fair, J. D.; Gora, T. F. Effect of Doping on the X-Ray Photoelectron Spectra of Semiconductors. Chem. Phys. Lett. 1971, 9 (6), 564-567. 
(10) Eberhardt, W.; Kalkoffen, G.; Kunz, C.; Aspnes, D.; Cardona, M. Photoemission Studies of $2 p$ Core Levels of Pure and Heavily Doped Silicon. Phys. Status Solidi B 1978, 88 (1), 135-143.

(11) Zhang, Z.; Yates, J. T., Jr. Effect of Adsorbed Donor and Acceptor Molecules on Electron Stimulated Desorption: $\mathrm{O}-2 / \mathrm{TiO}_{2}(110)$. J. Phys. Chem. Lett. 2010, 1 (14), 2185-2188.

(12) Kronik, L.; Shapira, Y. Surface Photovoltage Phenomena: Theory, Experiment, and Applications. Surf. Sci. Rep. 1999, 37 (1-5), 1-206.

(13) Powell, C. J.; Jablonski, A. Evaluation of Calculated and Measured Electron Inelastic Mean Free Paths near Solid Surfaces. J. Phys. Chem. Ref. Data 1999, 28 (1), 19-62.

(14) Hochella, M. F.; Carim, A. H. A Reassessment of Electron-Escape Depths in Silicon and Thermally Grown Silicon Dioxide Thin-Films. Surf. Sci. 1988, 197 (3), L260L268.

(15) Klasson, M.; Berndtsson, A.; Hedman, J.; Nilsson, R.; Nyholm, R.; Nordling, C. Electron Escape Depth in Silicon. J. Electron Spectrosc. Relat. Phenom. 1974, 3 (6), 427-434.

(16) Schlaf, R.; Hinogami, R.; Fujitani, M.; Yae, S.; Nakato, Y. Fermi Level Pinning on HF Etched Silicon Surfaces Investigated by Photoelectron Spectroscopy. J. Vac. Sci. Technol., A 1999, 17 (1), 164-169.

(17) Cohen, R.; Zenou, N.; Cahen, D.; Yitzchaik, S. Molecular Electronic Tuning of Si Surfaces. Chem. Phys. Lett. 1997, 279 (5-6), 270-274.

(18) Sezen, H.; Suzer, S. Dynamical XPS Measurements for Probing Photoinduced Voltage Changes. Surf. Sci. 2010, 604 (21-22), L59-L62.

(19) Ekiz, O. O.; Mizrak, K.; Dana, A. Chemically Specific Dynamic Characterization of Photovoltaic and Photoconductivity Effects of Surface Nanostructures. ACS Nano 2010, 4 (4), 1851-1860. 
(20) Hagimoto, Y.; Fujioka, H.; Oshima, M.; Hirose, K. Characterizing CarrierTrapping Phenomena in Ultrathin $\mathrm{SiO}_{2}$ Films by Using the X-Ray Photoelectron Spectroscopy Time-Dependent Measurements. Appl. Phys. Lett. 2000, 77 (25), 4175-4177.

(21) Himpsel, F. J.; Hollinger, G.; Pollak, R. A. Determination of the Fermi-Level Pinning Position at Si(111) Surfaces. Phys. Rev. B 1983, 28 (12), 7014-7018.

(22) Hagimoto, Y.; Fujita, T.; Ono, K.; Fujioka, H.; Oshima, M.; Hirose, K.; Tajima, M. Characterization of Carrier-Trapping Phenomena in Ultrathin Chemical Oxides Using X-Ray Photoelectron Spectroscopy Time-Dependent Measurements. Appl. Phys. Lett. 1999, 74 (14), 2011-2013.

(23) Song, W.; Yoshitake, M.; Tan, R.; Kojima, I. Band Bending and Band Alignment at $\mathrm{HfO}_{2} / \mathrm{HfSi}_{\mathrm{x}} \mathrm{O}_{\mathrm{y}} / \mathrm{Si}$ Interfaces. Appl. Surf. Sci. 2007, 253 (7), 3508-3511.

(24) Weiler, U.; Mayer, T.; Jaegermann, W.; Kelting, C.; Schlettwein, D.; Makarov, S.; Wohrle, D. Electronic Energy Levels of Organic Dyes on Silicon: A Photoelectron Spectroscopy Study of ZnPc, F16ZnPc, and ZnTPP on P-Si(111): H. J. Phys. Chem. B 2004, 108 (50), 19398-19403.

(25) Hunger, R.; Jaegermann, W.; Merson, A.; Shapira, Y.; Pettenkofer, C.; Rappich, J. Electronic Structure of Methoxy-, Bromo-, and Nitrobenzene Grafted onto Si(111). J. Phys. Chem. B 2006, 110 (31), 15432-15441.

(26) He, T.; Ding, H.; Peor, N.; Lu, M.; Corley, D. A.; Chen, B.; Ofir, Y.; Gao, Y.; Yitzchaik, S.; Tour, J. M. Silicon/Molecule Interfacial Electronic Modifications. J. Am. Chem. Soc. 2008, 130 (5), 1699-1710.

(27) He, T.; He, J.; Lu, M.; Chen, B.; Pang, H.; Reus, W. F.; Nolte, W. M.; Nackashi, D. P.; Franzon, P. D.; Tour, J. M. Controlled Modulation of Conductance in Silicon Devices by Molecular Monolayers. J. Am. Chem. Soc. 2006, 128 (45), 1453714541 .

(28) Cordier, S.; Fabre, B.; Molard, Y.; Fadjie-Djomkam, A.-B.; Tournerie, N.; Ledneva, A.; Naumov, N. G.; Moreac, A.; Turban, P.; Tricot, S.; Ababou-Girard, S.; Godet, C. Covalent Anchoring of $\mathrm{Re}_{6} \mathrm{Se}_{8}$ I Cluster Cores Mono Layers on Modified N- and PType Si(111) Surfaces: Effect of Coverage on Electronic Properties. J. Phys. Chem. C 2010, 114 (43), 18622-18633. 
(29) Papaefthimiou, V.; Kennou, S. Electronic Properties of a Semiconducting Oligomer/Silicon (111) Interface: Influence of Silicon Doping. Appl. Phys. Lett. 2005, $86(22)$.

(30) Hunger, R.; Fritsche, R.; Jaeckel, B.; Jaegermann, W.; Webb, L. J.; Lewis, N. S. Chemical and Electronic Characterization of Methyl-Terminated Si(111) Surfaces by High-Resolution Synchrotron Photoelectron Spectroscopy. Phys. Rev. B 2005, $72(4)$.

(31) Jaeckel, B.; Hunger, R.; Webb, L. J.; Jaegermann, W.; Lewis, N. S. High-Resolution Synchrotron Photoemission Studies of the Electronic Structure and Thermal Stability of $\mathrm{CH}_{3}$ - and $\mathrm{C}_{2} \mathrm{H}_{5}$-Functionalized $\mathrm{Si}(111)$ Surfaces. J. Phys. Chem. C 2007, 111 (49), 18204-18213.

(32) Hacker, C. A. Modifying Electronic Properties at the Silicon-Molecule Interface Using Atomic Tethers. Solid-State Electron. 2010, 54 (12), 1657-1664.

(33) Buriak, J. M. Organometallic Chemistry on Silicon and Germanium Surfaces. Chem. Rev. 2002, 102 (5), 1271-1308.

(34) Watanabe, D.; En, A.; Nakamura, S.; Suhara, M.; Okumura, T. Anomalously Large Band-Bending for HF-Treated P-Si Surfaces. Appl. Surf. Sci. 2003, 216 (1-4), 2429.

(35) Miyazaki, S.; Schafer, J.; Ristein, J.; Ley, L. Implication of Hydrogen-Induced Boron Passivation in Wet-Chemically Cleaned Si(111):H. Appl. Surf. Sci. 1997, $117,32-36$.

(36) Campbell, I. H.; Crone, B. K. Energy Level Alignments and Photocurrents in Crystalline Si/Organic Semiconductor Heterojunction Diodes. J. Appl. Phys. 2009, $106(11)$.

(37) Cooper, A. J.; Keyvanfar, K.; Deberardinis, A.; Pu, L.; Bean, J. C. Dopant Passivation and Work Function Tuning through Attachment of Heterogeneous Organic Monolayers on Silicon in Ultrahigh Vacuum. Appl. Surf. Sci. 2011, 257 (14), 6138-6144. 
(38) Sieval, A. B.; Huisman, C. L.; Schonecker, A.; Schuurmans, F. M.; van der Heide, A. S. H.; Goossens, A.; Sinke, W. C.; Zuilhof, H.; Sudholter, E. J. R. Silicon Surface Passivation by Organic Monolayers: Minority Charge Carrier Lifetime Measurements and Kelvin Probe Investigations. J. Phys. Chem. B 2003, 107 (28), 6846-6852.

(39) Bansal, A.; Li, X. L.; Lauermann, I.; Lewis, N. S.; Yi, S. I.; Weinberg, W. H. Alkylation of Si Surfaces Using a Two-Step Halogenation Grignard Route. J. Am. Chem. Soc. 1996, 118 (30), 7225-7226.

(40) Bansal, A.; Li, X. L.; Yi, S. I.; Weinberg, W. H.; Lewis, N. S. Spectroscopic Studies of the Modification of Crystalline Si(111) Surfaces with Covalently-Attached Alkyl Chains Using a Chlorination/Alkylation Method. J. Phys. Chem. B 2001, 105 (42), 10266-10277.

(41) Royea, W. J.; Juang, A.; Lewis, N. S. Preparation of Air-Stable, Low Recombination Velocity Si(111) Surfaces through Alkyl Termination. Appl. Phys. Lett. 2000, 77 (13), 1988-1990.

(42) Yu, H. B.; Webb, L. J.; Ries, R. S.; Solares, S. D.; Goddard, W. A.; Heath, J. R.; Lewis, N. S. Low-Temperature STM Images of Methyl-Terminated Si(111) Surfaces. J. Phys. Chem. B 2005, 109 (2), 671-674.

(43) Eves, B. J.; Lopinski, G. P. Formation and Reactivity of High Quality Halogen Terminated Si(111) Surfaces. Surf. Sci. 2005, 579 (2-3), L89-L96.

(44) Plass, K. E.; Liu, X. L.; Brunschwig, B. S.; Lewis, N. S. Passivation and Secondary Functionalization of Allyl-Terminated Si(111) Surfaces. Chem. Mater. 2008, 20 (6), 2228-2233.

(45) Fairley, N. Casaxps Manual 2.3.15: Spectroscopy; Casa Software Limited: Teignmouth, U.K., 2009.

(46) Schlaf, R. Calibration of Photoemission Spectra and Work Function Determination. http://rsl.eng.usf.edu/Documents/Tutorials/PEScalibration.pdf (accessed May 10, 2013). 
(47) Webb, L. J.; Rivillon, S.; Michalak, D. J.; Chabal, Y. J.; Lewis, N. S. Transmission Infrared Spectroscopy of Methyl- and Ethyl-Terminated Silicon(111) Surfaces. J. Phys. Chem. B 2006, 110 (14), 7349-7356.

(48) Dubey, G.; Rosei, F.; Lopinski, G. P. Modulation of Flat-Band Voltage on HTerminated Silicon-on-Insulator Pseudo-Metal-Oxide-Semiconductor Field Effect Transistors by Adsorption and Reaction Events. J. Appl. Phys. 2011, 109 (10).

(49) Shockley, W.; Queisser, H. J.; Hooper, W. W. Charges on Oxidized Silicon Surfaces. Phys. Rev. Lett. 1963, 11 (11), 489-490.

(50) Schlegel, E. S.; Schnable, G. L.; Schwarz, R. F.; Spratt, J. P. Behavior of Surface Ions on Semiconductor Devices. IEEE Trans. Electron Devices 1968, ED15 (12), 973. 